\title{
HARMONIC ANALYSIS OF ADDITIVE LÉVY PROCESSES
}

\author{
DAVAR KHOSHNEVISAN AND YIMIN XIAO
}

\begin{abstract}
Let $X_{1}, \ldots, X_{N}$ denote $N$ independent $d$-dimensional Lévy processes, and consider the $N$-parameter random field

$$
\mathfrak{X}(\boldsymbol{t}):=X_{1}\left(t_{1}\right)+\cdots+X_{N}\left(t_{N}\right) .
$$

First we demonstrate that for all nonrandom Borel sets $F \subseteq \mathbf{R}^{d}$, the Minkowski sum $\mathfrak{X}\left(\mathbf{R}_{+}^{N}\right) \oplus F$, of the range $\mathfrak{X}\left(\mathbf{R}_{+}^{N}\right)$ of $\mathfrak{X}$ with $F$, can have positive $d$-dimensional Lebesgue measure if and only if a certain capacity of $F$ is positive. This improves our earlier joint effort with Yuquan Zhong (2003) by removing a symmetry-type condition there. Moreover, we show that under mild regularity conditions, our necessary and sufficient condition can be recast in terms of one-potential densities. This rests on developing results in classical [non-probabilistic] harmonic analysis that might be of independent interest. As was shown in Khoshnevisan, Xiao, and Zhong (2003), the potential theory of the type studied here has a large number of consequences in the theory of Lévy processes. We present a few new consequences here.
\end{abstract}

\section{Introduction}

1.1. Background. It is known that, for all integers $d \geq 2$, the range of $d$-dimensional Brownian motion has zero Lebesgue measure. See Lévy (1940) for $d=2$, Ville (1942) for $d=3$, and Kakutani (1944a) for the remaining assertions. There is a perhaps betterknown, but equivalent, formulation of this theorem: When $d \geq 2$, the range of $d$-dimensional Brownian motion does not hit points. Kakutani (1944b) has generalized this by proving that, for all integers $d \geq 1$, the range of $d$-dimensional Brownian motion can hit a nonrandom Borel set $F \subseteq \mathbf{R}^{d}$ if and only if $\operatorname{cap}(F)>0$, where cap denotes, temporarily, the logarithmic capacity if $d=2$ and the Riesz capacity of index $d-2$ if $d \geq 3$; the case $d=1$ is elementary. [Actually, Kakutani's paper discusses only the planar case. The theorem, for $d \geq 3$, is pointed out in Dvoretzky, Erdős, and Kakutani (1950).]

Kakutani's theorem is the starting point of a deep probabilistic potential theory initiated by Hunt (1958, 1957a, 1957b, 1956). The literature on this topic is rich and quite large; see, for

Date: June 24, 2007.

1991 Mathematics Subject Classification. 60G60, 60J55, 60J45.

Key words and phrases. Additive Lévy processes, multiplicative Lévy processes, capacity, intersections of regenerative sets.

Research supported in part by a grant from the National Science Foundation. 
example, the books by Blumenthal and Getoor (1968), Doob (2001), Fukushima, Ōshima, and Takeda (19) Getoor (1990), and Röckner (1993), together with their combined bibliography.

One of the central assertions of probabilistic potential theory is that a nice Markov process will hit a nonrandom measurable set $F$ if and only if $\operatorname{cap}(F)>0$, where cap is a certain natural capacity in the sense of G. Choquet (Dellacherie and Meyer, 1978, Chapter III, pp. 51-55). Moreover, that capacity is defined solely, and fairly explicitly, in terms of the Markov process itself.

There are interesting examples where $F$ is itself random. For instance, suppose $X$ is $d$ dimensional standard Brownian motion, and $F=Y((0, \infty))$ is the range - minus the starting point — of an independent standard Brownian motion $Y$ on $\mathbf{R}^{d}$. In this particular case, it is well known that

$$
\mathrm{P}\{X(s)=Y(t) \text { for some } s, t>0\}>0 \text { if and only if } d \leq 3 .
$$

This result was proved by Lévy (1940) for $d=2$, Kakutani (1944a) for $d \geq 5$, and Dvoretzky, Erdős, and Ke for $d=3,4$. Peres (1996b, 1996a) and Khoshnevisan (2003) contain different elementary proofs of this fact.

There are many generalizations of (1.1) in the literature. For example, Dvoretzky, Erdős, and Kakutani proved that the paths of an arbitrary number of independent planar Brownian motions can intersect. While LeGall (1987) proved that the trajectories of a planar Brownian motion can intersect itself countably many times. And Dvoretzky, Erdős, Kakutani, and Taylor (1957) showed that three independent Brownian-motion trajectories in $\mathbf{R}^{d}$ can intersect if and only if $d \leq 2$. For other results along these lines, see Hawkes (1978b, 1977, 1976/77), Hendricks (1979, 1973/74), Kahane (1985, Chapter 16, Section 6), Lawler (1989, 1985, 1982), Pemantle, Peres, and Shapiro (1996), Le Gall (1992), Peres (1999, 1996b, 1996a), Rogers (1989), Tongring (1988), and their combined bibliography.

For a long time, a good deal of effort was concentrated on generalizing (1.1) to other concrete Markov processes than Brownian motion. But the problem of deciding when the paths of $N$ independent (general but nice) Markov processes can intersect remained elusive. It was finally settled, more than three decades later, by Fitzsimmons and Salisbury (1989), whose approach was to consider the said problem as one about a certain multiparameter Markov process.

To be concrete, let us consider the case $N=2$, and let $X:=\{X(t)\}_{t \geq 0}$ and $Y:=$ $\{Y(t)\}_{t \geq 0}$ denote two independent (nice) Markov processes on a (nice) state space $S$. The starting point of the work of Fitzsimmons and Salisbury is the observation that $\mathrm{P}\{X(s)=$ $Y(t)$ for some $s, t>0\}>0$ if and only if the two-parameter Markov process $X \otimes Y$ hits the 
diagonal diag $S:=\{x \otimes x: x \in S\}$ of $S \times S$, where

$$
(X \otimes Y)(s, t):=\left(\begin{array}{l}
X(s) \\
Y(t)
\end{array}\right) \quad \text { for all } s, t \geq 0 .
$$

In the special case that $X$ and $Y$ are Lévy processes, the Fitzsimmons-Salisbury theory was used to solve the then-long-standing Hendricks-Taylor conjecture (Hendricks and Taylor, 1979).

The said connection to multiparameter processes is of paramount importance in the Fitzsimmons-Salisbury theory, and appears earlier in the works of Evans (1987a, 1987b). See also Le Gall, Rosen, and Shieh (1989) and Salisbury (1996, 1992, 1988). Walsh (1986, pp. 364-368) discusses a connection between $X \otimes Y$ and the Dirichlet problem for the biLaplacian $\Delta \otimes \Delta$ on the bi-disc of $\mathbf{R}^{d} \times \mathbf{R}^{d}$.

The Fitzsimmons-Salisbury theory was refined and generalized in different directions by Hirsch (1995), Hirsch and Song (1999, 1996, 1995a, 1995b, 1995c, 1995d, 1994), and Khoshnevisan (2002, 1999). See also Ren (1990) who derives an implicit-function theorem in classical Wiener space by studying a very closely-related problem.

The two-parameter process $X \otimes Y$ itself was introduced earlier in the works of Wolpert (1978), who used $X \otimes Y$ to build a $\left(\phi^{\kappa}\right)_{2}$ model of the Euclidean field theory. This too initiated a very large body of works. For some of the earlier examples, see the works by Aizenman (1985), Albeverio and Zhou (1996), Dynkin (1987, 1986, 1985, 1984a, 1984b, 1984c, 1983a, 1983b, 1981, 1980), Felder and Fröhlich (1985), Rosen (1983, 1984), and Westwater (1980, 1981, 1982). [This is by no means an exhaustive list.]

In the case that $X$ and $Y$ are Lévy processes on $\mathbf{R}^{d}$ [i.e., have stationary independent increments], $X \otimes Y$ is an example of the so-called additive Lévy processes. But as it turns out, it is important to maintain a broader perspective and consider more than two Lévy processes. With this in mind, let $X_{1}, \ldots, X_{N}$ denote $N$ independent Lévy processes on $\mathbf{R}^{d}$ such that each $X_{j}$ is normalized via the Lévy-Khintchine formula (Bertoin, 1996; Sato, 1999):

$$
\mathrm{E} \exp \left(i \xi \cdot X_{j}(t)\right)=\exp \left(-t \Psi_{j}(\xi)\right) \quad \text { for all } t \geq 0 \text { and } \xi \in \mathbf{R}^{d} .
$$

The function $\Psi_{j}$ is called the characteristic exponent - or Lévy exponent - of $X_{j}$, and its defining property is that $\Psi_{j}$ is a negative-definite function (Schoenberg, 1938; Berg and Forst, 1975).

1.2. The main results. The main object of this paper is to develop some basic probabilistic potential theory for the following $N$-parameter random field $\mathfrak{X}$ with values in $\mathbf{R}^{d}$ :

$$
\mathfrak{X}(\boldsymbol{t}):=X_{1}\left(t_{1}\right)+\cdots+X_{N}\left(t_{N}\right) \quad \text { for all } \boldsymbol{t}:=\left(t_{1}, \ldots, t_{N}\right) \in \mathbf{R}_{+}^{N} .
$$

On a few occasions we might write $\left(\oplus_{j=1}^{N} X_{j}\right)(\boldsymbol{t})$ in place of $\mathfrak{X}(\boldsymbol{t})$, as well. 
The random field $\mathfrak{X}$ is a so-called additive Lévy process, and is characterized by its multiparameter Lévy-Khintchine formula:

$$
\mathrm{E} \exp (i \xi \cdot \mathfrak{X}(\boldsymbol{t}))=\exp (-\boldsymbol{t} \cdot \boldsymbol{\Psi}(\xi)) \quad \text { for all } \boldsymbol{t} \in \mathbf{R}_{+}^{N} \text { and } \xi \in \mathbf{R}^{d}
$$

where $\boldsymbol{\Psi}(\xi):=\left(\Psi_{1}(\xi), \ldots, \Psi_{N}(\xi)\right)$ is the characteristic exponent of $\mathfrak{X}$. Our goal is to describe the potential-theoretic properties of $\mathfrak{X}$ solely in terms of its characteristic exponent $\boldsymbol{\Psi}$. Thus, it is likely that our harmonic-analytic viewpoint can be extended to study the potential theory of more general multiparameter Markov processes that are based on the Feller processes of Jacob (2005, 2002, 2001).

In order to describe our main results let us first consider the kernel

$$
K_{\Psi}(\xi):=\prod_{j=1}^{N} \operatorname{Re}\left(\frac{1}{1+\Psi_{j}(\xi)}\right) \quad \text { for all } \xi \in \mathbf{R}^{d} .
$$

When $N=1$, this kernel plays a central role in the works of Orey (1967) and Kesten (1969).

The kernel for general $N$ was introduced first by Evans (1987b); see also Khoshnevisan, Xiao, and Zhong (2

Based on the kernel $K_{\Psi}$, we define, for all Schwartz distributions $\mu$ on $\mathbf{R}^{d}$,

$$
I_{\Psi}(\mu):=\frac{1}{(2 \pi)^{d}} \int_{\mathbf{R}^{d}}|\hat{\mu}(\xi)|^{2} K_{\Psi}(\xi) d \xi .
$$

We are primarily interested in the case where $\mu$ is a real-valued locally integrable function, or a $\sigma$-finite Borel measure on $\mathbf{R}^{d}$. In either case, we refer to $I_{\boldsymbol{\Psi}}(\mu)$ as the energy of $\mu$. Our notion of energy corresponds to a capacity cap $_{\boldsymbol{\Psi}}$, which is the following set function: For all Borel sets $F \subseteq \mathbf{R}^{d}$,

$$
\operatorname{cap}_{\Psi}(F):=\left[\inf _{\mu \in \mathscr{P}_{c}(F)} I_{\Psi}(\mu)\right]^{-1},
$$

where $\mathscr{P}_{c}(F)$ denotes the collection of all compactly-supported Borel probability measures on $F$, inf $\varnothing:=\infty$, and $1 / \infty:=0$.

The following is the first central result of this paper. Here and throughout, $\lambda_{k}$ denotes $k$-dimensional Lebesgue measure on $\mathbf{R}^{k}$ for all integers $k \geq 1$.

Theorem 1.1. Let $\mathfrak{X}$ be an $N$-parameter additive Lévy process on $\mathbf{R}^{d}$ with exponent $\boldsymbol{\Psi}$. Then, for all Borel sets $F \subseteq \mathbf{R}^{d}$,

$$
\mathrm{E}\left[\lambda_{d}\left(\mathfrak{X}\left(\mathbf{R}_{+}^{N}\right) \oplus F\right)\right]>0 \quad \text { if and only if } \operatorname{cap}_{\Psi}(F)>0 .
$$

Remark 1.2. (1) Theorem 1.1 in the one-parameter setting is still very interesting, but much easier to derive. See Kesten (1969) for the case that $F:=\{0\}$ and Hawkes (1984) for general $F$. For a scholarly pedagogic account see Bertoin (1996, p. 60). 
(2) One can view Theorem 1.1 as a contribution to the theory of Dirichlet forms for a class of infinite-dimensional Lévy processes. These Lévy processes are in general non-symmetric. Röckner (1993) describes a general theory of Dirichlet forms for nice infinite-dimensional Markov processes that are not necessarily symmetric. It would be interesting to know if the processes of the present paper lend themselves to the analysis of the general theory of Dirichlet forms. We have no conjectures along these lines.

Our earlier collaborative effort with Yuquan Zhong (2003) yielded the conclusion of Theorem 1.1 under an exogenous technical condition on $X_{1}, \ldots, X_{N}$. A first aim of this paper is to establish the fact that Theorem 1.1 holds in complete generality. Also, we showed in our earlier works (Khoshnevisan and Xiao, 2005; , 2004, Khoshnevisan, Xiao, and Zhong ; 2003) that such a theorem has a large number of consequences, many of them in the classical theory of Lévy processes itself. Next we describe a few such consequences that are nontrivial due to their intimate connections to harmonic analysis.

Our next result provides a criterion for a Borel set $F \subseteq \mathbf{R}^{d}$ to contain intersection points of $N$ independent Lévy processes. It completes and complements the well-known results of Fitzsimmons and Salisbury (1989). See also Corollary 9.3 and Remark 9.4 below.

Theorem 1.3. Let $X_{1}, \ldots, X_{N}$ be independent Lévy processes on $\mathbf{R}^{d}$, and assume that each $X_{j}$ has a one-potential density $u_{j}: \mathbf{R}^{d} \rightarrow \overline{\mathbf{R}}_{+}$such that $u_{j}(0)>0$. Then, for all nonempty Borel sets $F \subseteq \mathbf{R}^{d}$,

$$
\mathrm{P}\left\{X_{1}\left(t_{1}\right)=\cdots=X_{N}\left(t_{N}\right) \in F \text { for some } t_{1}, \ldots, t_{N}>0\right\}>0
$$

if and only if there exists a compact-support Borel probability measure $\mu$ on $F$ such that

$$
\int_{\mathbf{R}^{d}} \cdots \int_{\mathbf{R}^{d}}\left|\hat{\mu}\left(\xi^{1}+\cdots+\xi^{N}\right)\right|^{2} \prod_{j=1}^{N} \operatorname{Re}\left(\frac{1}{1+\Psi_{j}\left(\xi^{j}\right)}\right) d \xi^{1} \cdots d \xi^{N}<\infty .
$$

Suppose, in addition, that every $u_{j}$ is continuous on $\mathbf{R}^{d}$, and finite on $\mathbf{R}^{d} \backslash\{0\}$. Then, another equivalent condition is that there exists a compact-support probability measure $\mu$ on F such that

$$
\iint \prod_{j=1}^{N}\left(\frac{u_{j}(x-y)+u_{j}(y-x)}{2}\right) \mu(d x) \mu(d y)<\infty .
$$

In order to describe our next contribution, let us recall that the one-potential measure $U$ of a Lévy process $X:=\{X(t)\}_{t \geq 0}$ on $\mathbf{R}^{d}$ is defined as

$$
U(A):=\int_{0}^{\infty} \mathrm{P}\{X(t) \in A\} e^{-t} d t
$$


for all Borel sets $A \subseteq \mathbf{R}^{d}$. Next we offer a two-parameter "additive variant" which requires fewer technical conditions than Theorem 1.3 .

Theorem 1.4. Suppose $X_{1}$ and $X_{2}$ are independent Lévy processes on $\mathbf{R}^{d}$ with respective one-potential measures $U_{1}$ and $U_{2}$. Suppose $U_{1}(d x)=u_{1}(x) d x$, where $u_{1}: \mathbf{R}^{d} \rightarrow \overline{\mathbf{R}}_{+}$, and $u_{1} * U_{2}>0$ almost everywhere. Then, for all Borel sets $F \subseteq \mathbf{R}^{d}$,

$$
\mathrm{P}\left\{X_{1}\left(t_{1}\right)+X_{2}\left(t_{2}\right) \in F \text { for some } t_{1}, t_{2}>0\right\}>0
$$

if and only if there exists a compact-support Borel probability measure $\mu$ on $F$ such that

$$
\int_{\mathbf{R}^{d}}|\hat{\mu}(\xi)|^{2} \operatorname{Re}\left(\frac{1}{1+\Psi_{1}(\xi)}\right) \operatorname{Re}\left(\frac{1}{1+\Psi_{2}(\xi)}\right) d \xi<\infty .
$$

Suppose, in addition, that $u_{1}$ is continuous on $\mathbf{R}^{d}$, and finite on $\mathbf{R}^{d} \backslash\{0\}$. Then, (1.14) holds if and only if there exists a compact-support probability measure $\mu$ on $F$ such that

$$
\iint Q(x-y) \mu(d x) \mu(d y)<\infty
$$

where

$$
Q(x):=\int_{\mathbf{R}^{d}}\left[\frac{u_{1}(x+y)+u_{1}(x-y)+u_{1}(-x+y)+u_{1}(-x-y)}{4}\right] U_{2}(d y)
$$

for all $x \in \mathbf{R}^{d}$.

Among other things, Theorem 1.4 confirms a conjecture of Bertoin (1999b) and Bertoin (1999a, p. 49); see Remark 8.1 for details.

Finally we mention a result on the Hausdorff dimension of the set of intersections of the sample paths of Lévy processes.

Theorem 1.5. Let $X_{1}, \ldots, X_{N}$ be independent Lévy processes on $\mathbf{R}^{d}$, and assume that each $X_{j}$ has a one-potential density $u_{j}: \mathbf{R}^{d} \rightarrow \overline{\mathbf{R}}_{+}$such that $u_{j}(0)>0$. Then, almost surely on $\left\{\cap_{k=1}^{N} X_{k}\left(\mathbf{R}_{+}\right) \neq \varnothing\right\}$,

$$
\begin{aligned}
& \operatorname{dim}_{\mathrm{H}} \bigcap_{k=1}^{N} X_{k}\left(\mathbf{R}_{+}\right) \\
& \quad=\sup \left\{s \in(0, d): \int_{\left(\mathbf{R}^{d}\right)^{N}} \prod_{j=1}^{N} \operatorname{Re}\left(\frac{1}{1+\Psi_{j}\left(\xi^{j}\right)}\right) \frac{d \xi}{1+\left\|\xi^{1}+\cdots+\xi^{N}\right\|^{d-s}}<\infty\right\},
\end{aligned}
$$

where $\sup \varnothing:=0$. Suppose, in addition, that the $u_{j}$ 's are continuous on $\mathbf{R}^{d}$, and finite on $\mathbf{R}^{d} \backslash\{0\}$. Then, almost surely on $\left\{\cap_{k=1}^{N} X_{k}\left(\mathbf{R}_{+}\right) \neq \varnothing\right\}$,

$$
\operatorname{dim}_{\mathrm{H}} \bigcap_{k=1}^{N} X_{k}\left(\mathbf{R}_{+}\right)=\sup \left\{s \in(0, d): \int_{\mathbf{R}^{d}} \prod_{j=1}^{N}\left(\frac{u_{j}(z)+u_{j}(-z)}{2}\right) \frac{d z}{\|z\|^{s}}<\infty\right\} .
$$


In the remainder of the paper we prove Theorem 1.1 and its stated corollaries in the order in which they are presented. Finally, we conclude by two zero-one laws for the Lebesgue measure and capacity of the range of an additive Lévy process, that, we believe, might have independent interest.

We end this section with four problems and conjectures.

Open problem 1. Throughout this paper, we impose continuity conditions on various onepotential densities. This is mainly because we are able to develop general harmonic-analytic results only for kernels that satisfy some regularity properties. Can the continuity conditions be dropped? We believe the answer is "yes." This is motivated, in part, by the following fact, which follows from inspecting the proofs: The condition " $u$ is continuous on $\mathbf{R}^{d}$ and finite on $\mathbf{R}^{d} \backslash\{0\}$ " is used only for proving the "if" portions in the second parts of Theorems 1.3 and 1.4 .

Open problem 2. Jacob (2005; 2002, 2001) has constructed a very large class of Feller processes that behave locally like Lévy processes. Moreover, his construction is deeply connected to harmonic analysis. Because the results of the present paper involve mainly the local structure of Lévy processes, and are inextricably harmonic analytic, we ask: Is it possible to study the harmonic-analytic potential theory of several Jacob processes by somehow extending the methods of the present paper?

Open problem 3. We ask: Is there a "useful" theory of excessive functions and/or measures for additive Lévy processes (or more general multiparameter Markov processes)? This question is intimately connected to Open Problem 1, but deserves to be asked on its own. In the one-parameter case, the answer is a decisive "yes" (Getoor, 1990). But the one-dimensional theory does not appear to readily have a suitable extension to the multiparameter setting.

Open problem 4. We conjecture that, under the conditions of Theorem 1.5, the following holds almost surely on $\left\{\cap_{k=1}^{N} X_{k}\left(\mathbf{R}_{+}\right) \neq \varnothing\right\}$ :

$$
\operatorname{dim}_{\mathrm{H}} \bigcap_{k=1}^{N} X_{k}\left(\mathbf{R}_{+}\right)=\sup \left\{s \in(0, d): \int_{(-1,1)^{d}} \prod_{j=1}^{N}\left(\frac{u_{j}(z)+u_{j}(-z)}{2}\right) \frac{d z}{\|z\|^{s}}<\infty\right\} .
$$

[The difference between this and (1.19) is in the range of the integrals.] But in all but one case we have no proof; see Remark 9.6 below for the mentioned case. As we shall see in that remark, what we actually prove is the following harmonic-analytic fact: Suppose $u$ is the one-potential density of a Lévy process, $u(0)>0, u$ is continuous on $\mathbf{R}^{d}$, and $u$ is finite on $\mathbf{R}^{d} \backslash\{0\}$. Then the local square-integrability of $u$ implies the [global] square-integrability of $u$. We believe that the following more general result holds: If $u_{1}, \ldots, u_{N}$ are one-potential 
densities that share the stated properties for $u$, then

$$
\prod_{j=1}^{N}\left(\frac{u_{j}(\bullet)+u_{j}(-\bullet)}{2}\right) \in L_{l o c}^{1}\left(\mathbf{R}^{d}\right) \Rightarrow \prod_{j=1}^{N}\left(\frac{u_{j}(\bullet)+u_{j}(-\bullet)}{2}\right) \in L^{1}\left(\mathbf{R}^{d}\right) .
$$

If this is so, then the results of this paper imply Conjecture (1.20).

\section{The stationary additive Lévy random field}

Consider a classical Lévy process $X:=\{X(t)\}_{t \geq 0}$ on $\mathbf{R}^{d}$ with characteristic exponent $\Psi$. Let us introduce an independent copy $X^{\prime}$ of $X$, and extend the definition of $X$ to a process indexed by $\mathbf{R}$ as follows:

$$
\tilde{X}(t):= \begin{cases}X(t) & \text { if } t \geq 0 \\ -X^{\prime}(-t) & \text { if } t<0 .\end{cases}
$$

This is the two-sided Lévy process with exponent $\Psi$ in the sense that $\widetilde{X}:=\{\tilde{X}(t)\}_{t \in \mathbf{R}}$ has stationary and independent increments. Moreover, $\{\widetilde{X}(t+s)-\widetilde{X}(s)\}_{t \geq 0}$ is a copy of $X$ for all $s \in \mathbf{R}$.

We also define $\widetilde{\mathfrak{X}}:=\{\widetilde{\mathfrak{X}}(\boldsymbol{t})\}_{\boldsymbol{t} \in \mathbf{R}^{N}}$ as the corresponding $N$-parameter process, indexed by all of $\mathbf{R}^{N}$, whose values are in $\mathbf{R}^{d}$ and are defined as

$$
\widetilde{\mathfrak{X}}(\boldsymbol{t}):=\widetilde{X}_{1}\left(t_{1}\right)+\cdots+\widetilde{X}_{N}\left(t_{N}\right) \quad \text { for all } \boldsymbol{t}:=\left(t_{1}, \ldots, t_{N}\right) \in \mathbf{R}^{N} \text {. }
$$

We are assuming, of course, that $\widetilde{X}_{1}, \ldots, \widetilde{X}_{N}$ are independent two-sided extensions of the processes $X_{1}, \ldots, X_{N}$, respectively.

We intend to prove the following two-sided version of Theorem 1.1.

Theorem 2.1. For all Borel sets $F \subseteq \mathbf{R}^{d}$,

$$
\mathrm{E}\left[\lambda_{d}\left(\widetilde{\mathfrak{X}}\left(\mathbf{R}^{N}\right) \oplus F\right)\right]>0 \quad \text { if and only if } \operatorname{cap}_{\mathbf{\Psi}}(F)>0 \text {. }
$$

This implies Theorem 1.1 effortlessly. Indeed, we know already from Remark 1.2 of Khoshnevisan, Xiao, and Zhong (2003) that

$$
\operatorname{cap}_{\mathbf{\Psi}}(F)>0 \quad \Longrightarrow \quad \mathrm{E}\left[\lambda_{d}\left(\mathfrak{X}\left(\mathbf{R}_{+}^{N}\right) \oplus F\right)\right]>0 \text {. }
$$

Thus, we seek only to derive the converse implication. But that follows from Theorem 2.1 , because $\mathfrak{X}\left(\mathbf{R}_{+}^{N}\right) \subseteq \widetilde{\mathfrak{X}}\left(\mathbf{R}^{N}\right)$.

Henceforth, we assume that the underlying probability space is the collection of all paths $\omega: \mathbf{R}^{N} \rightarrow \mathbf{R}^{d}$ that have the form $\omega(\boldsymbol{t})=\sum_{j=1}^{N} \omega_{j}\left(t_{j}\right)$ for all $\boldsymbol{t} \in \mathbf{R}^{N}$, where each $\omega_{j}$ maps $\mathbf{R}^{N}$ to $\mathbf{R}^{d}$ such that $\omega_{j}(\mathbf{0})=0$; and $\omega_{j} \in D_{\mathbf{R}^{d}}(\mathbf{R})$, the Skorohod space of cadlag functions from $\mathbf{R}$-not $[0, \infty)$ - to $\mathbf{R}^{d}$. 
We can then assume that the stationary additive Lévy fields, described earlier in this section, are in canonical form. That is, $\widetilde{\mathfrak{X}}(\boldsymbol{t})(\omega):=\omega(\boldsymbol{t})$ for all $t \in \mathbf{R}^{N}$ and $\omega \in \Omega$. Because we are interested only in distributional results, this is a harmless assumption.

Define $\mathrm{P}_{x}$ to be the law of $x+\widetilde{\mathfrak{X}}$, and $\mathrm{E}_{x}$ the expectation operation with respect to $\mathrm{P}_{x}$, for every $x \in \mathbf{R}^{d}$. Thus, we are identify $\mathrm{P}$ with $\mathrm{P}_{0}$, and $\mathrm{E}$ with $\mathrm{E}_{0}$.

We are interested primarily in the $\sigma$-finite measure

$$
\mathrm{P}_{\lambda_{d}}:=\int_{\mathbf{R}^{d}} \mathrm{P}_{x} d x
$$

and the corresponding expectation operator $\mathrm{E}_{\lambda_{d}}$, defined by

$$
\mathrm{E}_{\lambda_{d}} f:=\int_{\Omega} f(\omega) \mathrm{P}_{\lambda_{d}}(d \omega) \quad \text { for all } f \in L^{1}\left(\mathrm{P}_{\lambda_{d}}\right) .
$$

If $A \ominus B:=\{a-b: a \in A, b \in B\}$, then by the Fubini-Tonelli theorem,

$$
\begin{aligned}
\mathrm{E}\left[\lambda_{d}\left(\widetilde{\mathfrak{X}}\left(\mathbf{R}^{N}\right) \ominus F\right)\right] & =\mathrm{E}\left[\int_{\mathbf{R}^{d}} \mathbf{1}_{\widetilde{\mathfrak{X}}\left(\mathbf{R}^{N}\right) \ominus F}(x) d x\right] \\
& =\int_{\mathbf{R}^{d}} \mathrm{P}_{-x}\left\{\widetilde{\mathfrak{X}}(\boldsymbol{t}) \in F \text { for some } \boldsymbol{t} \in \mathbf{R}^{N}\right\} d x \\
& =\mathrm{P}_{\lambda_{d}}\left\{\widetilde{\mathfrak{X}}\left(\mathbf{R}^{N}\right) \cap F \neq \varnothing\right\} .
\end{aligned}
$$

Thus, Theorem 2.1 is a potential-theoretic characterization of all polar sets for $\widetilde{\mathfrak{X}}$ under the $\sigma$-finite measure $\mathrm{P}_{\lambda_{d}}$. With this viewpoint in mind, we proceed to introduce some of the fundamental objects that are related to the process $\widetilde{\mathfrak{X}}$.

Define, for all $\boldsymbol{t} \in \mathbf{R}^{N}$, the linear operator $P_{\boldsymbol{t}}$ as follows:

$$
\left(P_{\boldsymbol{t}} f\right)(x):=\mathrm{E}_{x}[f(\widetilde{\mathfrak{X}}(\boldsymbol{t}))] \quad \text { for all } x \in \mathbf{R}^{d} .
$$

This is well-defined, for example, if $f: \mathbf{R}^{d} \rightarrow \mathbf{R}_{+}$is Borel-measurable, or if $f: \mathbf{R}^{d} \rightarrow \mathbf{R}$ is Borel-measurable and $P_{\boldsymbol{t}}(|f|)$ is finite at $x$. Also define the linear operator $R$ by

$$
(R f)(x):=\frac{1}{2^{N}} \int_{\mathbf{R}^{N}}\left(P_{\boldsymbol{t}} f\right)(x) e^{-[\boldsymbol{t}]} d \boldsymbol{t} \quad \text { for all } x \in \mathbf{R}^{d},
$$

where

$$
[\boldsymbol{t}]:=\left|t_{1}\right|+\cdots+\left|t_{N}\right|
$$

denotes the $\ell^{1}$-norm of $\boldsymbol{t} \in \mathbf{R}^{N}$. [We will use this notation throughout.] The $\ell^{2}$-norm of $\boldsymbol{t} \in \mathbf{R}^{N}$ will be denoted by $\|\boldsymbol{t}\|$.

Again, $(R f)(x)$ is well defined if $f: \mathbf{R}^{d} \rightarrow \mathbf{R}_{+}$is Borel-measurable, or if $f: \mathbf{R}^{d} \rightarrow \mathbf{R}$ is Borel-measurable and $R(|f|)$ is finite at $x$. 
Our next result is a basic regularity lemma for $R$. It should be recognized as a multiparameter version of a very well-known property of Markovian semigroups and their resolvents.

Lemma 2.2. Each $P_{\boldsymbol{t}}$ and $R$ are contractions on $L^{p}\left(\mathbf{R}^{d}\right)$, as long as $1 \leq p \leq \infty$.

Proof. Choose and fix $j \in\{1, \ldots, N\}$ and $t \in \mathbf{R}$, and define $\mu_{j, t}$ to be the distribution of the random variable $-\widetilde{X}_{j}(t)$. If $f: \mathbf{R}^{d} \rightarrow \mathbf{R}_{+}$is Borel-measurable, then

$$
P_{\boldsymbol{t}} f=f * \mu_{1, t_{1}} * \cdots * \mu_{N, t_{N}},
$$

where $*$ denotes convolution. This implies readily that $P_{\boldsymbol{t}}$ is a contraction on $L^{p}\left(\mathbf{R}^{d}\right)$ for all $p \in[1, \infty]$, and hence,

$$
\|R f\|_{L^{p}\left(\mathbf{R}^{d}\right)} \leq \frac{1}{2^{N}} \int_{\mathbf{R}^{N}}\left\|P_{\boldsymbol{t}} f\right\|_{L^{p}\left(\mathbf{R}^{d}\right)} e^{-[t]} d \boldsymbol{t} .
$$

Since $P_{\boldsymbol{t}}$ is a contraction on $L^{p}\left(\mathbf{R}^{d}\right)$, the preceding is bounded above by $\|f\|_{L^{p}\left(\mathbf{R}^{d}\right)}$.

Henceforth, let "^" denote the [Schwartz] Fourier transform on any and every Euclidean space $\mathbf{R}^{k}$. Our Fourier transform is normalized such that

$$
\hat{f}(\xi):=\int_{\mathbf{R}^{k}} e^{i \xi \cdot x} f(x) d x,
$$

for all $f \in L^{1}\left(\mathbf{R}^{k}\right)$, say. We then have the following.

Lemma 2.3. If $f \in L^{1}\left(\mathbf{R}^{d}\right)$, then $\hat{R}=K_{\Psi}$. That is, $\widehat{(R f)}(\xi)=K_{\Psi}(\xi) \hat{f}(\xi)$ for all $\xi \in \mathbf{R}^{d}$.

Proof. Recall $\mu_{j, t}$ from the proof of Lemma 2.2. Its Fourier transform is given by

$$
\widehat{\mu_{j, t}}(\xi)=\exp \left\{-|t| \Psi_{j}(-\operatorname{sgn}(t) \xi)\right\} \quad \text { for all } \xi \in \mathbf{R}^{d} .
$$

Equations (2.11) and (2.14), and the Plancherel theorem together imply that

$$
\widehat{\left(P_{t} f\right)}(\xi)=\hat{f}(\xi) \exp \left(-\sum_{j=1}^{N}\left|t_{j}\right| \Psi_{j}\left(-\operatorname{sgn}\left(t_{j}\right) \xi\right)\right) \text {. }
$$

Consequently,

$$
\begin{aligned}
\widehat{(R f)}(\xi) & =\frac{1}{2^{N}} \hat{f}(\xi) \int_{\mathbf{R}^{N}} \exp \left(-\sum_{j=1}^{N}\left|t_{j}\right|\left[1+\Psi_{j}\left(-\operatorname{sgn}\left(t_{j}\right) \xi\right)\right]\right) d \boldsymbol{t} \\
& =\frac{1}{2^{N}} \hat{f}(\xi) \prod_{j=1}^{N} \int_{0}^{\infty}\left(e^{-t\left[1+\Psi_{j}(\xi)\right]}+e^{-t\left[1+\Psi_{j}(-\xi)\right]}\right) d t .
\end{aligned}
$$

A direct computation reveals that $\widehat{R f}=K_{\Psi} \hat{f}$, as asserted.

The following is a functional-analytic consequence. 
Corollary 2.4. The operator $R$ maps $L^{2}\left(\mathbf{R}^{d}\right)$ into $L^{2}\left(\mathbf{R}^{d}\right)$, and is self-adjoint.

Proof. By Lemma 2.3, if $f \in L^{1}\left(\mathbf{R}^{d}\right) \cap L^{2}\left(\mathbf{R}^{d}\right)$ and $g \in L^{2}\left(\mathbf{R}^{d}\right)$, then

$$
\int_{\mathbf{R}^{d}}(R f)(x) g(x) d x=\frac{1}{(2 \pi)^{d}} \int_{\mathbf{R}^{d}} K_{\mathbf{\Psi}}(\xi) \hat{f}(\xi) \overline{\hat{g}(\xi)} d \xi .
$$

Thanks to Lemma 2.2, the preceding holds for all $f \in L^{2}\left(\mathbf{R}^{d}\right)$. Duality then implies that $R: L^{2}\left(\mathbf{R}^{d}\right) \rightarrow L^{2}\left(\mathbf{R}^{d}\right)$. Moreover, since $K_{\Psi}$ is real, $R$ is self-adjoint.

The following lemma shows that for every $\boldsymbol{t} \in \mathbf{R}^{N}$, the distribution of $\widetilde{\mathfrak{X}}(\boldsymbol{t})$ under $\mathrm{P}_{\lambda}$ is $\lambda_{d}$. This is the reason why we call $\widetilde{\mathfrak{X}}$ a stationary additive Lévy process.

Lemma 2.5. If $f: \mathbf{R}^{d} \rightarrow \mathbf{R}_{+}$is Borel-measurable, then

$$
\mathrm{E}_{\lambda_{d}}[f(\widetilde{\mathfrak{X}}(\boldsymbol{t}))]=\int_{\mathbf{R}^{d}}\left(P_{\boldsymbol{t}} f\right)(x) d x=\int_{\mathbf{R}^{d}} f(y) d y \quad \text { for all } \boldsymbol{t} \in \mathbf{R}^{N} .
$$

Proof. We apply the Fubini-Tonelli theorem to find that

$$
\mathrm{E}_{\lambda_{d}}[f(\widetilde{\mathfrak{X}}(\boldsymbol{t}))]=\mathrm{E} \int_{\mathbf{R}^{d}} f(x+\widetilde{\mathfrak{X}}(\boldsymbol{t})) d x .
$$

A change of variables $[y:=x+\widetilde{\mathfrak{X}}(\boldsymbol{t})]$ proves that the preceding expression is equal to the integral of $f$. This implies half of the lemma. Another application of the Fubini-Tonelli theorem implies the remaining half as well.

Let us choose and fix $\pi \subseteq\{1, \ldots, N\}$ and identify $\pi$ with the partial order $\prec_{\pi}$, on $\mathbf{R}^{N}$, which is defined as follows: For all $s, \boldsymbol{t} \in \mathbf{R}^{N}$,

$$
s \prec_{\pi} \boldsymbol{t} \quad \text { iff } \quad \begin{cases}s_{i} \leq t_{i} & \text { for all } i \in \pi, \text { and } \\ s_{i}>t_{i} & \text { for all } i \notin \pi .\end{cases}
$$

The collection of all $\pi \subseteq\{1, \ldots, N\}$ forms a collective total order on $\mathbf{R}^{N}$ in the sense that

$$
\text { for all } \boldsymbol{s}, \boldsymbol{t} \in \mathbf{R}^{N} \text { there exists } \pi \subseteq\{1, \ldots, N\} \text { such that } \boldsymbol{s} \prec_{\pi} \boldsymbol{t} \text {. }
$$

For all $\pi \subseteq\{1, \ldots, N\}$, we define the $\pi$-history of the random field $\widetilde{\mathfrak{X}}$ as the collection

$$
\mathscr{H}_{\pi}(\boldsymbol{t}):=\sigma\left(\{\widetilde{\mathfrak{X}}(s)\}_{s \prec \pi}\right) \quad \text { for all } \boldsymbol{t} \in \mathbf{R}^{N},
$$

where $\sigma(\cdots)$ denotes the $\sigma$-algebra generated by whatever is in the parentheses. Without loss of generality, we assume that each $\mathscr{H}_{\pi}(\boldsymbol{t})$ is complete with respect to $\mathrm{P}_{x}$ for all $x \in \mathbf{R}^{d}$; else, we replace it with the said completion. Also, we assume without loss of generality that $\boldsymbol{t} \mapsto \mathscr{H}_{\pi}(\boldsymbol{t})$ is $\pi$-right-continuous. More precisely, we assume that

$$
\mathscr{H}_{\pi}(\boldsymbol{t})=\bigcap_{\boldsymbol{s} \in \mathbf{R}^{N}: \boldsymbol{t} \prec_{\pi} \boldsymbol{s}} \mathscr{H}_{\pi}(\boldsymbol{s}) \quad \text { for all } \boldsymbol{t} \in \mathbf{R}^{N} \text { and } \pi \subseteq\{1, \ldots, N\} .
$$


If not, then we replace the left-hand side by the right-hand side everywhere.

Proposition 2.6 (A Markov-random-field property). Suppose $\pi \subseteq\{1, \ldots, N\}$ and $\boldsymbol{s} \prec_{\pi} \boldsymbol{t}$, both in $\mathbf{R}^{N}$. Then, for all measurable functions $f: \mathbf{R}^{d} \rightarrow \mathbf{R}_{+}$,

$$
\mathrm{E}_{\lambda_{d}}\left[f(\widetilde{\mathfrak{X}}(\boldsymbol{t})) \mid \mathscr{H}_{\pi}(\boldsymbol{s})\right]=\left(P_{\boldsymbol{t}-\boldsymbol{s}} f\right)(\widetilde{\mathfrak{X}}(\boldsymbol{s})) \quad \mathrm{P}_{\lambda_{d}} \text {-a.s. }
$$

Proof. Choose and fix Borel measurable functions $g, \phi_{1}, \ldots, \phi_{m}: \mathbf{R}^{d} \rightarrow \mathbf{R}_{+}$, and " $N$ parameter time points" $\boldsymbol{s}^{1}, \ldots, \boldsymbol{s}^{m} \in \mathbf{R}^{N}$ such that

$$
\boldsymbol{s}^{j} \prec_{\pi} s \prec_{\pi} \boldsymbol{t} \quad \text { for all } j=1, \ldots, m \text {. }
$$

According to the Fubini-Tonelli theorem,

$$
\begin{aligned}
\mathrm{E}_{\lambda_{d}}[f(\widetilde{\mathfrak{X}}(\boldsymbol{t})) g(\widetilde{\mathfrak{X}}(\boldsymbol{s})) & \left.\prod_{j=1}^{m} \phi_{j}\left(\widetilde{\mathfrak{X}}\left(\boldsymbol{s}^{j}\right)\right)\right] \\
& =\int_{\mathbf{R}^{d}} \mathrm{E}\left[f(x+\widetilde{\mathfrak{X}}(\boldsymbol{t})) g(x+\widetilde{\mathfrak{X}}(\boldsymbol{s})) \prod_{j=1}^{m} \phi_{j}\left(x+\widetilde{\mathfrak{X}}\left(\boldsymbol{s}^{j}\right)\right)\right] d x \\
& =\int_{\mathbf{R}^{d}} \mathrm{E}\left[f(A+y) \prod_{j=1}^{m} \phi_{j}\left(A_{j}+y\right)\right] g(y) d y
\end{aligned}
$$

where $A:=\widetilde{\mathfrak{X}}(\boldsymbol{t})-\widetilde{\mathfrak{X}}(\boldsymbol{s})$ and $A_{j}:=\widetilde{\mathfrak{X}}\left(\boldsymbol{s}^{j}\right)-\widetilde{\mathfrak{X}}(\boldsymbol{s})$ for all $j=1, \ldots, m$.

The independent-increments property of each of the Lévy processes $\widetilde{X}_{j}$ implies that $A$ is independent of $\left\{A_{j}\right\}_{j=1}^{m}$. This and the stationary-increments property of $\widetilde{X}_{1}, \ldots, \widetilde{X}_{N}$ together imply that

$$
\begin{aligned}
\mathrm{E}_{\lambda_{d}}\left[f(\widetilde{\mathfrak{X}}(\boldsymbol{t})) g(\widetilde{\mathfrak{X}}(\boldsymbol{s})) \prod_{j=1}^{m} \phi_{j}\left(\widetilde{\mathfrak{X}}\left(\boldsymbol{s}^{j}\right)\right)\right] \\
=\int_{\mathbf{R}^{d}} \mathrm{E}[f(A+y)] \mathrm{E}\left[\prod_{j=1}^{m} \phi_{j}\left(A_{j}+y\right)\right] g(y) d y .
\end{aligned}
$$


After a change of variables and an appeal to the stationary-independent property of the increments of $X_{1}, \ldots, X_{N}$ and $X_{1}^{\prime}, \ldots, X_{N}^{\prime}$, we arrive at the following:

$$
\begin{aligned}
\mathrm{E}_{\lambda_{d}}\left[f(\widetilde{\mathfrak{X}}(\boldsymbol{t})) g(\widetilde{\mathfrak{X}}(\boldsymbol{s})) \prod_{j=1}^{m} \phi_{j}\left(\widetilde{\mathfrak{X}}\left(\boldsymbol{s}^{j}\right)\right)\right] \\
=\int_{\mathbf{R}^{d}} \mathrm{E}[f(\widetilde{\mathfrak{X}}(\boldsymbol{t}-\boldsymbol{s})+y)] \mathrm{E}_{y}\left[\prod_{j=1}^{m} \phi_{j}\left(A_{j}\right)\right] g(y) d y \\
=\int_{\mathbf{R}^{d}}\left(P_{\boldsymbol{t}-\boldsymbol{s}} f\right)(y) \mathrm{E}_{y}\left[\prod_{j=1}^{m} \phi_{j}\left(A_{j}\right)\right] g(y) d y .
\end{aligned}
$$

By the monotone class theorem, for all nonnegative $\mathscr{H}_{\pi}(\boldsymbol{s})$-measurable random variables $Y$,

$$
\mathrm{E}_{\lambda_{d}}[f(\widetilde{\mathfrak{X}}(\boldsymbol{t})) g(\widetilde{\mathfrak{X}}(\boldsymbol{s})) Y]=\int_{\mathbf{R}^{d}}\left(P_{\boldsymbol{t}-\boldsymbol{s}} f\right)(y) \psi(y) g(y) d y,
$$

where $\psi: \mathbf{R}^{d} \rightarrow \mathbf{R}_{+}$is a measurable function. This proves the proposition.

Lemma 2.7. If $f, g \in L^{2}\left(\mathbf{R}^{d}\right)$ and $\boldsymbol{t}, \boldsymbol{s} \in \mathbf{R}^{N}$, then

$$
\mathrm{E}_{\lambda_{d}}[f(\widetilde{\mathfrak{X}}(\boldsymbol{s})) g(\widetilde{\mathfrak{X}}(\boldsymbol{t}))]=\int_{\mathbf{R}^{d}} f(y)\left(P_{\boldsymbol{t}-\boldsymbol{s}} g\right)(y) d y .
$$

Proof. We may consider, without loss of generality, measurable and nonnegative functions $f, g \in L^{2}\left(\mathbf{R}^{d}\right)$. Let $\pi$ denote the collection of all $i \in\{1, \ldots, N\}$ such that $s_{i} \leq t_{i}$. Then $\boldsymbol{s} \prec_{\pi} \boldsymbol{t}$, and Proposition 2.6 implies that $\mathrm{P}_{\lambda_{d}}$-a.s.,

$$
\mathrm{E}_{\lambda_{d}}\left[f(\widetilde{\mathfrak{X}}(\boldsymbol{t})) \mid \mathscr{H}_{\pi}(\boldsymbol{s})\right]=\left(P_{\boldsymbol{t}-\boldsymbol{s}} f\right)(\widetilde{\mathfrak{X}}(\boldsymbol{s})) .
$$

This and Lemma 2.5 together conclude the proof.

\section{The sojourn operator}

Recall (2.10), and consider the "sojourn operator,"

$$
S f:=\frac{1}{2^{N}} \int_{\mathbf{R}^{N}} f(\widetilde{\mathfrak{X}}(\boldsymbol{t})) e^{-[\boldsymbol{t}]} d \boldsymbol{t} .
$$

Our first lemma records the fact that $S$ maps density functions to mean-one random variables $\left[\mathrm{P}_{\lambda_{d}}\right]$.

Lemma 3.1. If $f$ is a probability density function on $\mathbf{R}^{d}$, then $\mathrm{E}_{\lambda_{d}}[S f]=1$.

This follows readily from Lemma 2.5. Our next result shows that, under a mild condition on $\Psi_{j}, S$ embeds functions in $L^{2}\left(\mathbf{R}^{d}\right)$, quasi-isometrically, into the subcollection of all functions in $L^{2}\left(\mathbf{R}^{d}\right)$ that have finite energy. Namely, 
Proposition 3.2. If $f \in L^{2}\left(\mathbf{R}^{d}\right)$, then

$$
\|S f\|_{L^{2}\left(\mathrm{P}_{\lambda_{d}}\right)} \leq \sqrt{I_{\Psi}(f)} .
$$

Suppose, in addition, that there exists a constant $c \in(0, \sqrt{2})$, such that the following sector condition holds for all $j=1, \ldots, N$ :

$$
\left|\operatorname{Im} \Psi_{j}(\xi)\right| \leq c\left(1+\operatorname{Re} \Psi_{j}(\xi)\right) \quad \text { for all } \xi \in \mathbf{R}^{d} .
$$

Then, there exists a constant $A \in(0,1)$ such that

$$
A \sqrt{I_{\Psi}(f)} \leq\|S f\|_{L^{2}\left(\mathrm{P}_{\lambda_{d}}\right)} \leq \sqrt{I_{\Psi}(f)} .
$$

Remark 3.3 (Generalized Sobolev spaces). When $N=1$ and the Lévy process in question is symmetric, the following problem arises in the theory of Dirichlet forms: For what $f$ in the class $\mathscr{D}\left(\mathbf{R}^{d}\right)$, of Schwartz distributions on $\mathbf{R}^{d}$, can we define $S f$ as an element of $L^{2}\left(\mathrm{P}_{\lambda_{d}}\right)$ (say)? This problem continues to make sense in the more general context of additive Lévy processes. And the answer is given by (3.4) in Proposition 3.2 as follows: Assume that the sector condition (3.3) holds for all $j=1, \ldots, N$ and some $c \in(0, \sqrt{2})$. Let $\mathscr{S}_{\boldsymbol{\Psi}}\left(\mathbf{R}^{d}\right)$ denote the completion of the collection of all members of $L^{2}\left(\mathbf{R}^{d}\right)$ that have finite energy $I_{\Psi}$, where the completion is made in the norm $\|f\|_{\Psi}:=I_{\Psi}^{1 / 2}(f)+\|f\|_{L^{2}\left(\mathbf{R}^{d}\right)}$. Then, there exists an a.s.-unique maximal extension $\bar{S}$ of $S$ such that $\bar{S}: \mathscr{S}_{\Psi}\left(\mathbf{R}^{d}\right) \rightarrow \bar{S}\left(\mathscr{S}_{\Psi}\left(\mathbf{R}^{d}\right)\right)$ is a quasi-isometry. The space $\mathscr{S}_{\mathbf{\Psi}}\left(\mathbf{R}^{d}\right)$ generalizes further some of the $\psi$-Bessel potential spaces of Farkas, Jacob, and Schilling (2001) and Farkas and Leopold (2006); see also Jacob and Schilling (2005), Masja and Nagel (1978), and Slobodeckiǔ (1958).

The proof requires a technical lemma, which we develop first.

Lemma 3.4. For all $z \in \mathrm{C}$ define

$$
\Lambda(z):=\int_{-\infty}^{\infty} \int_{-\infty}^{\infty} e^{-|t|-|s|-|t-s| \sigma(z ; t-s)} d t d s,
$$

where $\sigma(z ; r):=z$ if $r \geq 0$ and $\sigma(z ; r):=\bar{z}$ otherwise. Then for all $z \in \mathbf{C}$ with $\operatorname{Re} z \geq 0$,

$$
\Lambda(z) \leq 4 \operatorname{Re}\left(\frac{1}{1+z}\right) .
$$

If, in addition, $|\operatorname{Im} z| \leq c(1+\operatorname{Re} z)$ for some $c \in(0, \sqrt{2})$, then

$$
\Lambda(z) \geq 2\left(2-c^{2}\right) \operatorname{Re}\left(\frac{1}{1+z}\right) .
$$

Proof. The double integral is computed by dividing the region of integration into four natural parts: (i) $s, t \geq 0$; (ii) $s, t \leq 0$; (iii) $t \geq 0 \geq s$; and (iv) $s \geq 0 \geq t$. Direct computation 
reveals that for all $z \in \mathbf{C}$ with $\operatorname{Re} z \geq 0$

$$
\begin{aligned}
\int_{0}^{\infty} \int_{0}^{\infty} e^{-|t|-|s|-|t-s| \sigma(z ; t-s)} d t d s+\int_{-\infty}^{0} \int_{-\infty}^{0} e^{-|t|-|s|-|t-s| \sigma(z ; t-s)} d t d s & \\
& =2 \operatorname{Re}\left(\frac{1}{1+z}\right) .
\end{aligned}
$$

Similarly, one can compute

$$
\begin{aligned}
\int_{0}^{\infty} \int_{-\infty}^{0} e^{-|t|-|s|-|t-s| \sigma(z ; t-s)} d t d s+\int_{-\infty}^{0} \int_{0}^{\infty} e^{-|t|-|s|-|t-s| \sigma(z ; t-s)} d t d s & =\frac{1}{(1+z)^{2}}+\frac{1}{(1+\bar{z})^{2}}
\end{aligned}
$$

Consequently,

$$
\Lambda(z)=2 \operatorname{Re}\left(\frac{1}{1+z}\right)+\frac{2\left((1+\operatorname{Re} z)^{2}-(\operatorname{Im} z)^{2}\right)}{|1+z|^{4}},
$$

for all $z \in \mathbf{C}$ with $\operatorname{Re} z \geq 0$. It follows that

$$
\Lambda(z) \leq 2 \operatorname{Re}\left(\frac{1}{1+z}\right)\left[1+\operatorname{Re}\left(\frac{1}{1+z}\right)\right]
$$

for all $z \in \mathbf{C}$ with $\operatorname{Re} z \geq 0$. Whenever $\operatorname{Re} z \geq 0$, we have $0 \leq \operatorname{Re}(1+z)^{-1} \leq 1$, and hence (3.6) follows from (3.11). On the other hand, if $|\operatorname{Im} z| \leq c(1+\operatorname{Re} z)$, then (3.10) yields

$$
\Lambda(z) \geq 2 \operatorname{Re}\left(\frac{1}{1+z}\right)+2\left(1-c^{2}\right)\left[\operatorname{Re}\left(\frac{1}{1+z}\right)\right]^{2},
$$

from which the result follows readily, because $0 \leq \operatorname{Re}\left[(1+z)^{-1}\right] \leq 1$ when $\operatorname{Re} z \geq 0$.

Proof of Proposition 3.2. We apply Lemma 2.7 to deduce that

$$
\mathrm{E}_{\lambda_{d}}\left(|S f|^{2}\right)=\frac{1}{4^{N}} \int_{\mathbf{R}^{d}} \iint_{\mathbf{R}^{N} \times \mathbf{R}^{N}} e^{-[t]-[\boldsymbol{s}]} f(y)\left(P_{\boldsymbol{t}-\boldsymbol{s}} f\right)(y) d \boldsymbol{t} d \boldsymbol{s} d y .
$$

In accord with (2.15) and Parseval's identity, for all $\boldsymbol{u} \in \mathbf{R}^{N}$,

$$
\begin{aligned}
\int_{\mathbf{R}^{d}} f(y)\left(P_{\boldsymbol{u}} f\right)(y) d y & =\frac{1}{(2 \pi)^{d}} \int_{\mathbf{R}^{d}} \overline{\hat{f}(\xi)} \widehat{\left(P_{\boldsymbol{u}} f\right)}(\xi) d \xi \\
& =\frac{1}{(2 \pi)^{d}} \int_{\mathbf{R}^{d}}|\hat{f}(\xi)|^{2} \exp \left(-\sum_{j=1}^{N}\left|u_{j}\right| \Psi_{j}\left(-\operatorname{sgn}\left(u_{j}\right) \xi\right)\right) d \xi .
\end{aligned}
$$

This and the Fubini-Tonelli theorem together reveal that

$$
\mathrm{E}_{\lambda_{d}}\left(|S f|^{2}\right)=\frac{1}{4^{N}(2 \pi)^{d}} \int_{\mathbf{R}^{d}}|\hat{f}(\xi)|^{2} \prod_{j=1}^{N} \Lambda\left(\Psi_{j}(\xi)\right) d \xi .
$$


Since $\operatorname{Re} \Psi_{j}(\xi) \geq 0$ for all $\xi \in \mathbf{R}^{d}$ and $j=1, \ldots, N$, we apply Lemma 3.4 to this formula, and conclude the proof of the proposition.

\section{Proof of Theorem 2.1}

Thanks to the definition of $\operatorname{cap}_{\boldsymbol{\Psi}}$, and to the countable additivity of $\mathrm{P}$, it suffices to consider only the case that

$$
F \text { is a compact set. }
$$

This condition is tacitly assumed throughout this section. We note, in particular, that $\mathscr{P}_{c}(F)$ denotes merely the collection of all Borel probability measures that are supported on $F$.

Proposition 5.7 of Khoshnevisan, Xiao, and Zhong (2003) proves that for every compact set $F \subseteq \mathbf{R}^{d}$

$$
\operatorname{cap}_{\Psi}(F)>0 \Longrightarrow \mathrm{E}\left[\lambda_{d}\left(\mathfrak{X}\left([0, r]^{N}\right) \oplus F\right)\right]>0
$$

for all $r>0$. It is clear the latter implies that $\mathrm{E}\left[\lambda_{d}\left(\widetilde{\mathfrak{X}}\left(\mathbf{R}^{N}\right) \oplus F\right)\right]>0$, and one obtains half of the theorem.

Since $\operatorname{cap}_{\boldsymbol{\Psi}}(-F)=\operatorname{cap}_{\boldsymbol{\Psi}}(F)$, we may and will replace $F$ by $-F$ throughout. In light of (2.7), we then assume that

$$
\mathrm{P}_{\lambda_{d}}\left\{\widetilde{\mathfrak{X}}\left(\mathbf{R}^{N}\right) \cap F \neq \varnothing\right\}>0
$$

and seek to deduce the existence of a probability measure $\mu$ on $F$ such that $I_{\Psi}(\mu)<\infty$.

We will prove a little more. Namely, that for all $k>0$ there exists a constant $A=$ $A(k, N) \in(0, \infty)$ such that

$$
\mathrm{P}_{\lambda_{d}}\left\{\widetilde{\mathfrak{X}}\left([-k, k]^{N}\right) \cap F \neq \varnothing\right\} \leq A \operatorname{cap}_{\Psi}(F) .
$$

In fact, we will prove that for all sufficiently large $k>0$,

$$
\mathrm{P}_{\lambda_{d}}\left\{\widetilde{\mathfrak{X}}\left([-k, k]^{N}\right) \cap F \neq \varnothing\right\} \leq e^{2 N k} 4^{N} \operatorname{cap}_{\boldsymbol{\Psi}}(F) .
$$

This would conclude our proof of the second half of the theorem.

It is a standard fact that there exists a probability density function $\phi_{1}$ in $C^{\infty}\left(\mathbf{R}^{d}\right)$ with the following properties:

P1. $\phi_{1}(x)=0$ if $\|x\|>1$;

P2. $\phi_{1}(x)=\phi_{1}(-x)$ for all $x \in \mathbf{R}^{d}$;

P3. $\hat{\phi}_{1}(x) \geq 0$ for all $x \in \mathbf{R}^{d}$.

This can be obtained, for example, readily from Plancherel's [duality] theorem of Fourier analysis. 
We recall also the following standard fact: $\phi_{1} \in L^{1}\left(\mathbf{R}^{d}\right)$ and $\mathbf{P} 3$ together imply that $\hat{\phi}_{1} \in L^{1}\left(\mathbf{R}^{d}\right)$ (Hawkes, 1984, Lemma 1).

Now we define an approximation to the identity $\left\{\phi_{\epsilon}\right\}_{\epsilon>0}$ by setting

$$
\phi_{\epsilon}(x):=\frac{1}{\epsilon^{d}} \phi_{1}\left(\frac{x}{\epsilon}\right) \quad \text { for all } x \in \mathbf{R}^{d} \text { and } \epsilon>0 .
$$

It follows readily from this that for every $\mu \in \mathscr{P}_{c}(F)$ :

(i) $\mu * \phi_{\epsilon}$ is a uniformly continuous probability density;

(ii) $\mu * \phi_{\epsilon}$ is supported on the closed $\epsilon$-enlargement of $F$, which we denote by $F_{\epsilon}$, for all $\epsilon>0$

(iii) $\phi_{\epsilon}$ is symmetric; and

(iv) $\lim _{\epsilon \rightarrow 0^{+}} \hat{\phi}_{\epsilon}(\xi)=1$ for all $\xi \in \mathbf{R}^{d}$.

As was done in Khoshnevisan, Xiao, and Zhong (2003), we can find a random variable $\boldsymbol{T}$ with values in $\mathbf{R}^{N} \cup\{\infty\}$ such that:

(1) $\{\boldsymbol{T}=\infty\}$ is equal to the event that $\widetilde{\mathfrak{X}}(\boldsymbol{t}) \notin F$ for all $\boldsymbol{t} \in \mathbf{R}^{N}$;

(2) $\widetilde{\mathfrak{X}}(\boldsymbol{T}) \in F$ on $\{\boldsymbol{T} \neq \infty\}$.

This can be accomplished pathwise. Consequently, (4.3) is equivalent to the condition that

$$
\mathrm{P}_{\lambda_{d}}\{\boldsymbol{T} \neq \infty\}>0
$$

Define for all Borel sets $A \subseteq \mathbf{R}^{d}$ and all integers $k \geq 1$,

$$
\mu_{k}(A):=\mathrm{P}_{\lambda_{d}}\left(\widetilde{\mathfrak{X}}(\boldsymbol{T}) \in A \mid \boldsymbol{T} \in[-k, k]^{N}\right) .
$$

We claim that $\mu_{k}$ is a probability measure on $F$ for all $k$ sufficiently large. In order to prove this claim we choose and fix $l>0$, and consider

$$
\mu_{k, l}(A):=\frac{\mathrm{P}_{\lambda_{d}}\left\{\widetilde{\mathfrak{X}}(\boldsymbol{T}) \in A, \boldsymbol{T} \in[-k, k]^{N},|\widetilde{\mathfrak{X}}(\mathbf{0})| \leq l\right\}}{\mathrm{P}_{\lambda_{d}}\left\{\boldsymbol{T} \in[-k, k]^{N},|\widetilde{\mathfrak{X}}(\mathbf{0})| \leq l\right\}} .
$$

Because the $\mathrm{P}_{\lambda_{d}}$-distribution of $\widetilde{\mathfrak{X}}(\mathbf{0})$ is $\lambda_{d}, \mu_{k, l}$ is a probability measure on $F$ for all $k$ and $l$ sufficiently large; see (4.7). And $\mu_{k, l}(A)$ converges to $\mu_{k}(A)$ for all Borel sets $A$ as $l \uparrow \infty$ [monotone convergence theorem]. This proves the assertion that $\mu_{k} \in \mathscr{P}_{c}(F)$ for all $k$ large. Choose and fix such a large integer $k$. 
Now we define $f_{\epsilon}:=\mu_{k} * \phi_{\epsilon}$, and observe that according to Proposition 2.6, for all nonrandom times $\boldsymbol{\tau} \in \mathbf{R}^{N}$,

$$
\begin{aligned}
\sum_{\pi \subseteq\{1, \ldots, N\}} \mathrm{E}_{\lambda_{d}}\left[\int_{\boldsymbol{t} \succ \pi} f_{\epsilon}(\widetilde{\mathfrak{X}}(\boldsymbol{t})) e^{-[t]} d \boldsymbol{t} \mid \mathscr{H}_{\pi}(\boldsymbol{\tau})\right] \\
=\sum_{\pi \subseteq\{1, \ldots, N\}} \int_{\boldsymbol{t}_{\pi} \boldsymbol{\tau}}\left(P_{\boldsymbol{t}-\boldsymbol{\tau}} f_{\epsilon}\right)(\widetilde{\mathfrak{X}}(\boldsymbol{\tau})) e^{-[t]} d \boldsymbol{t} \\
\geq e^{-[\boldsymbol{\tau}]} \sum_{\pi \subseteq\{1, \ldots, N\}} \int_{\boldsymbol{s \succ \pi} \mathbf{0}}\left(P_{\boldsymbol{s}} f_{\epsilon}\right)(\widetilde{\mathfrak{X}}(\boldsymbol{\tau})) e^{-[\boldsymbol{s}]} d \boldsymbol{s} .
\end{aligned}
$$

We can apply (2.21) to deduce then that for all nonrandom times $\boldsymbol{\tau} \in \mathbf{R}^{N}$,

$$
\begin{aligned}
\mathrm{E}_{\lambda_{d}}\left[S f_{\epsilon} \mid \mathscr{H}_{\pi}(\boldsymbol{\tau})\right] & \geq \frac{e^{-[\boldsymbol{\tau}]}}{2^{N}} \int_{\mathbf{R}^{N}}\left(P_{\boldsymbol{s}} f_{\epsilon}\right)(\widetilde{\mathfrak{X}}(\boldsymbol{\tau})) e^{-[\boldsymbol{s}]} d \boldsymbol{s} \\
& =e^{-[\boldsymbol{\tau}]}\left(R f_{\epsilon}\right)(\widetilde{\mathfrak{X}}(\boldsymbol{\tau})) .
\end{aligned}
$$

Because $f_{\epsilon}$ is continuous and compactly supported, one can verify from (2.9) that $R f_{\epsilon}$ is continuous [this can also be shown by Lemma 2.3, the fact that $\hat{f}_{\epsilon} \in L^{1}\left(\mathbf{R}^{d}\right)$ and the Fourier inversion formula]. Also, the $L^{2}\left(\mathrm{P}_{\lambda_{d}}\right)$-norm of the left-most term in (4.11) is bounded above by $\left\|S f_{\epsilon}\right\|_{L^{2}\left(\mathrm{P}_{\lambda_{d}}\right)}$, and this is at most $\sqrt{I_{\Psi}\left(f_{\epsilon}\right)}$, in turn; see Proposition 3.2. It is easy to see that

$$
\begin{aligned}
I_{\Psi}\left(f_{\epsilon}\right) & =I_{\Psi}\left(\mu_{k} * \phi_{\epsilon}\right) \\
& \leq \frac{1}{(2 \pi)^{2 d}} \int_{\mathbf{R}^{d}}\left|\hat{\phi}_{\epsilon}(\xi)\right|^{2} d \xi \\
& <\infty .
\end{aligned}
$$

Since $\left|\hat{\mu}_{k}(\xi)\right|^{2} K_{\Psi}(\xi) \leq 1$, we conclude that $\left\|S f_{\epsilon}\right\|_{L^{2}\left(\mathrm{P}_{\lambda_{d}}\right)}$ is finite for all $\epsilon>0$.

A simple adaptation of the proof of Proposition 2.6 proves that $\mathscr{H}_{\pi}$ is a commuting filtration for all $\pi \subseteq\{1, \ldots, N\}$. By this we mean that for all $\boldsymbol{t} \in \mathbf{R}$,

$$
\mathscr{H}_{\pi}^{1}\left(t_{1}\right), \ldots, \mathscr{H}_{\pi}^{N}\left(t_{N}\right) \text { are conditionally independent }\left[\mathrm{P}_{\lambda_{d}}\right] \text {, given } \mathscr{H}_{\pi}(\boldsymbol{t}),
$$

where $\mathscr{H}_{\pi}^{j}\left(t_{j}\right)$ is defined as the following $\sigma$-algebra:

$$
\mathscr{H}_{\pi}^{j}\left(t_{j}\right):= \begin{cases}\sigma\left(\widetilde{X}_{j}(s) ;-\infty<s \leq t_{j}\right) & \text { if } j \in \pi, \\ \sigma\left(\widetilde{X}_{j}(s) ; \infty>s \geq t_{j}\right) & \text { if } j \notin \pi .\end{cases}
$$

Here, $\sigma(\cdots)$ denotes the $\sigma$-algebra generated by the random variables in the parenthesis.

The stated commutation property readily implies that for all random variables $Y \in$ $L^{2}\left(\mathrm{P}_{\lambda_{d}}\right)$ and partial orders $\pi \subseteq\{1, \ldots, N\}$ : 
(1) $\boldsymbol{\tau} \mapsto \mathrm{E}_{\lambda_{d}}\left[Y \mid \mathscr{H}_{\pi}(\boldsymbol{\tau})\right]$ has a version that is cadlag in each of its $N$ variables, uniformly in all other $N-1$ variables; and

(2) The second moment of $\sup _{\boldsymbol{\tau} \in \mathbf{R}^{N}} \mathrm{E}_{\lambda_{d}}\left[Y \mid \mathscr{H}_{\pi}(\boldsymbol{\tau})\right]$ is at most $4^{N}$ times the second moment of $Y\left[\mathrm{P}_{\lambda_{d}}\right]$.

See Khoshnevisan (2002, Theorem 2.3.2, p. 235) for the case where $\mathrm{P}_{\lambda_{d}}$ is replaced by a probability measure. The details of the remaining changes are explained in a slightly different setting in Lemma 4.2 of Khoshnevisan, Xiao, and Zhong (2003). In summary, (4.11) holds for all $\boldsymbol{\tau} \in \mathbf{R}^{N}, \mathrm{P}_{\lambda_{d}}$-almost surely [note the order of the quantifiers]. It follows immediately from this that for all integers $k \geq 1$,

$$
\sup _{\boldsymbol{\tau} \in \mathbf{R}^{N}} \mathrm{E}_{\lambda_{d}}\left[S f_{\epsilon} \mid \mathscr{H}_{\pi}(\boldsymbol{\tau})\right] \geq e^{-N k}\left(R f_{\epsilon}\right)(\widetilde{\mathfrak{X}}(\boldsymbol{T})) \cdot \mathbf{1}_{\left\{\boldsymbol{T} \in[-k, k]^{N}\right\}} \quad \mathrm{P}_{\lambda_{d}-\text { a.s. }}
$$

According to Item (2) above, the second moment of the left-hand side is at most $4^{N}$ times the second moment of $S f_{\epsilon}$. As we noticed earlier, the latter is at most $I_{\Psi}\left(f_{\epsilon}\right)$. Therefore,

$$
\begin{aligned}
e^{2 N k} 4^{N} I_{\boldsymbol{\Psi}}\left(f_{\epsilon}\right) & \geq \mathrm{E}_{\lambda_{d}}\left[\left|\left(R f_{\epsilon}\right)(\widetilde{\mathfrak{X}}(\boldsymbol{T}))\right|^{2} ; \boldsymbol{T} \in[-k, k]^{N}\right] \\
& =\mathrm{E}_{\lambda_{d}}\left[\left|\left(R f_{\epsilon}\right)(\widetilde{\mathfrak{X}}(\boldsymbol{T}))\right|^{2} \mid \boldsymbol{T} \in[-k, k]^{N}\right] \cdot \mathrm{P}_{\lambda_{d}}\left\{\boldsymbol{T} \in[-k, k]^{N}\right\} .
\end{aligned}
$$

It follows from this and the Cauchy-Schwarz inequality that

$$
e^{2 N k} 4^{N} I_{\boldsymbol{\Psi}}\left(f_{\epsilon}\right) \geq\left|\mathrm{E}_{\lambda_{d}}\left[\left(R f_{\epsilon}\right)(\widetilde{\mathfrak{X}}(\boldsymbol{T})) \mid \boldsymbol{T} \in[-k, k]^{N}\right]\right|^{2} \cdot \mathrm{P}_{\lambda_{d}}\left\{\boldsymbol{T} \in[-k, k]^{N}\right\} .
$$

Using the definition of $\mu_{k}$, we can write the above as

$$
\begin{aligned}
e^{2 N k} 4^{N} I_{\boldsymbol{\Psi}}\left(f_{\epsilon}\right) & \geq\left|\int_{\mathbf{R}^{d}}\left(R f_{\epsilon}\right) d \mu_{k}\right|^{2} \cdot \mathrm{P}_{\lambda_{d}}\left\{\boldsymbol{T} \in[-k, k]^{N}\right\} \\
& \left.=\frac{1}{(2 \pi)^{2 d}} \mid \int_{\mathbf{R}^{d}} \overline{\hat{\mu}_{k}(\xi)} \widehat{\left(R f_{\epsilon}\right.}\right)\left.(\xi) d \xi\right|^{2} \cdot \mathrm{P}_{\lambda_{d}}\left\{\boldsymbol{T} \in[-k, k]^{N}\right\},
\end{aligned}
$$

where the equality follows from the Parseval identity. According to Lemma 2.3, the Fourier transform of $R f_{\epsilon}$ is $K_{\Psi}$ times the Fourier transform of $f_{\epsilon}$, and the latter is $\hat{\mu}_{k} \hat{\phi}_{\epsilon}$. Hence (4.18) implies that

$$
e^{2 N k} 4^{N} I_{\Psi}\left(f_{\epsilon}\right) \geq\left.\left.\frac{1}{(2 \pi)^{2 d}}\left|\int_{\mathbf{R}^{d}}\right| \hat{\mu}_{k}(\xi)\right|^{2} \hat{\phi}_{\epsilon}(\xi) K_{\Psi}(\xi) d \xi\right|^{2} \cdot \mathrm{P}_{\lambda_{d}}\left\{\boldsymbol{T} \in[-k, k]^{N}\right\} .
$$


Now we apply Property P3 to deduce that $\hat{\phi}_{\epsilon} \geq 0$. Because $\phi_{\epsilon}$ is also a probability density, it follows that $\hat{\phi}_{\epsilon} \geq\left|\hat{\phi}_{\epsilon}\right|^{2}$. Consequently,

$$
\begin{aligned}
e^{2 N k} 4^{N} I_{\boldsymbol{\Psi}}\left(f_{\epsilon}\right) & \geq\left.\left.\frac{1}{(2 \pi)^{2 d}}\left|\int_{\mathbf{R}^{d}}\right| \hat{f}_{\epsilon}(\xi)\right|^{2} K_{\boldsymbol{\Psi}}(\xi) d \xi\right|^{2} \cdot \mathrm{P}_{\lambda_{d}}\left\{\boldsymbol{T} \in[-k, k]^{N}\right\} \\
& =\left|I_{\boldsymbol{\Psi}}\left(f_{\epsilon}\right)\right|^{2} \cdot \mathrm{P}_{\lambda_{d}}\left\{\boldsymbol{T} \in[-k, k]^{N}\right\} .
\end{aligned}
$$

We have seen in (4.12) that $I_{\Psi}\left(f_{\epsilon}\right)$ is finite for each $\epsilon>0$. If it were zero for arbitrary small $\epsilon>0$, then we apply Fatou's lemma to deduce that $I_{\Psi}\left(\mu_{k}\right) \leq \liminf _{\epsilon \rightarrow 0} I_{\Psi}\left(f_{\epsilon}\right)=0$. This and the fact that $K_{\boldsymbol{\Psi}}(\xi)>0$ for all $\xi \in \mathbf{R}^{d}$ would imply $\hat{\mu}_{k}(\xi) \equiv 0$ for all $\xi \in \mathbf{R}^{d}$, which is a contradiction. Hence we can deduce that for all $\epsilon>0$ small enough,

$$
\frac{e^{2 N k} 4^{N}}{I_{\Psi}\left(f_{\epsilon}\right)} \geq \mathrm{P}_{\lambda_{d}}\left\{\boldsymbol{T} \in[-k, k]^{N}\right\}
$$

and this is positive for $k$ large; see (4.7). The right-hand side of (4.21) is independent of $\epsilon>0$. Hence, we can let $\epsilon \downarrow 0$ and appeal to Fatou's lemma to deduce that $I_{\Psi}\left(\mu_{k}\right)<\infty$. Thus, in any event, we have produced a probability measure $\mu_{k}$ on $F$ whose energy $I_{\Psi}\left(\mu_{k}\right)$ is finite. This concludes the proof, and also implies (4.5), thanks to the defining properties of the function $\boldsymbol{T}$.

\section{On kernels of positive type}

In this section we study kernels of positive type; they are recalled next. Here and throughout, $\overline{\mathbf{R}}_{+}:=[0, \infty]$ is defined to be the usual one-point compactification of $\mathbf{R}_{+}:=[0, \infty)$, and is endowed with the corresponding Borel sigma-algebra.

Definition 5.1. A kernel [on $\mathbf{R}^{d}$ ] is a Borel measurable function $\kappa: \mathbf{R}^{d} \rightarrow \overline{\mathbf{R}}_{+}$such that $\kappa \in L_{\text {loc }}^{1}\left(\mathbf{R}^{d}\right)$. If, in addition, $\hat{\kappa}(\xi) \geq 0$ for all $\xi \in \mathbf{R}^{d}$, then we say that $\kappa$ is a kernel of positive type.

Clearly, every kernel $\kappa$ can be redefined on a Lebesgue-null set so that the resulting modification $\tilde{\kappa}$ maps $\mathbf{R}^{d}$ into $\mathbf{R}_{+}$. However, we might lose some of the nice properties of $\kappa$ by doing this. A notable property is that $\kappa$ might be continuous; that is, $\kappa(x) \rightarrow \kappa(y)-$ in $\overline{\mathbf{R}}_{+}$-as $x$ converges to $y$ in $\mathbf{R}^{d}$. In this case, $\tilde{\kappa}$ might not be continuous. From this perspective, it is sometimes advantageous to work with the $\overline{\mathbf{R}}_{+}$-valued function $\kappa$. For examples, we have in mind Riesz kernels. They are defined as follows: Choose and fix some number $\alpha \in(0, d)$, and then let the Riesz kernel $\kappa_{\alpha}$ of index $\alpha$ be

$$
\kappa_{\alpha}(x):= \begin{cases}\|x\|^{\alpha-d} & \text { if } x \neq 0 \\ \infty & \text { if } x=0\end{cases}
$$


It is easy to check that $\kappa_{\alpha}$ is a continuous kernel for each $\alpha \in(0, d)$. In fact, every $\kappa_{\alpha}$ is a kernel of positive type, as can be seen via the following standard fact:

$$
\hat{\kappa}_{\alpha}(\xi)=c_{d, \alpha} \kappa_{d-\alpha}(\xi) \quad \text { for all } \xi \in \mathbf{R}^{d} .
$$

Here $c_{d, \alpha}$ is a universal constant that depends only on $d$ and $\alpha$; see Kahane (1985, p. 134) or Mattila (1995, eq. (12.10), p. 161), for example.

It is true - but still harder to prove - that for all Borel probability measures $\mu$ on $\mathbf{R}^{d}$,

$$
\iint \frac{\mu(d x) \mu(d y)}{\|x-y\|^{d-\alpha}}=\frac{c_{d, \alpha}}{(2 \pi)^{d}} \int_{\mathbf{R}^{d}}|\hat{\mu}(\xi)|^{2} \frac{d \xi}{\|\xi\|^{\alpha}} .
$$

See Mattila (1995, p. Lemma 12.12, p. 162).

The utility of (5.3) is in the fact that it shows that Riesz-type energies of the left-hand side are equal to Pólya-Szegő energies of the right-hand side. This is a probabilistically significant fact. For example, consider the case that $\alpha \in(0,2]$. Then, $\mu \mapsto \iint\|x-y\|^{-d+\alpha} \mu(d x) \mu(d y)$ is the "energy functional" associated to continuous additive functionals of various stable processes of index $\alpha$. At the same time, $\mu \mapsto(2 \pi)^{-d} \int_{\mathbf{R}^{d}}|\hat{\mu}(\xi)|^{2}\|\xi\|^{-\alpha} d \xi$ is a Fourier-analytic energy form of the type that appears more generally in the earlier parts of the present paper. Roughly speaking, $(2 \pi)^{-d} \int_{\mathbf{R}^{d}}|\hat{\mu}(\xi)|^{2}\|\xi\|^{-\alpha} d \xi \asymp I_{\Psi}(\mu)$, where $\Psi(\xi)=\|\xi\|^{\alpha}$ defines the Lévy exponent of an isotropic stable process of index $\alpha$. [Analytically speaking, this is the Sobolev norm of $\mu$ that corresponds to the fractional Laplacian operator $-(-\Delta)^{\alpha / 2}$.] Thus, we seek to find a useful generalization of (5.3) that goes beyond one-parameter stable processes.

We define for all finite Borel measures $\mu$ and $\nu$ on $\mathbf{R}^{d}$, and all kernels $\kappa$ on $\mathbf{R}^{d}$,

$$
\mathscr{E}_{\kappa}(\mu, \nu):=\iint\left(\frac{\kappa(x-y)+\kappa(y-x)}{2}\right) \mu(d x) \nu(d y) .
$$

This is called the mutual energy between $\mu$ and $\nu$ in gauge $\kappa$, and defines a quadratic form with pseudo-norm

$$
\mathscr{E}_{\kappa}(\mu):=\mathscr{E}_{\kappa}(\mu, \mu)
$$

This is the " $\kappa$-energy" of the measure $\mu$. There is a corresponding capacity defined as

$$
\mathscr{C}_{\kappa}(F):=\frac{1}{\inf \mathscr{E}_{\kappa}(\mu)},
$$

where the infimum is taken over all compactly supported probability measures $\mu$ on $F$, $\inf \varnothing:=\infty$, and $1 / \infty:=0$.

We can recognize the left-hand side of $(\underline{5.3})$ to be $\mathscr{E}_{\kappa_{d-\alpha}}(\mu)$. Because $\kappa_{d-\alpha}(x)<\infty$ if and only if $x \neq 0$, the following is a nontrivial generalization of (5.3). 
Theorem 5.2. Suppose $\kappa$ is a continuous kernel of positive type on $\mathbf{R}^{d}$ which satisfies one of the following two conditions:

(1) $\kappa(x)<\infty$ if and only if $x \neq 0$;

(2) $\hat{\kappa} \in L^{\infty}\left(\mathbf{R}^{d}\right)$, and $\kappa(x)<\infty$ when $x \neq 0$.

Then for all Borel probability measures $\mu$ and $\nu$ on $\mathbf{R}^{d}$,

$$
\mathscr{E}_{\kappa * \nu}(\mu)=\frac{1}{(2 \pi)^{d}} \int_{\mathbf{R}^{d}} \hat{\kappa}(\xi) \operatorname{Re} \hat{\nu}(\xi)|\hat{\mu}(\xi)|^{2} d \xi .
$$

Remark 5.3. If $\kappa \in L^{1}\left(\mathbf{R}^{d}\right)$, then $\hat{\kappa}$ can be define by the usual Fourier transform, $\hat{\kappa}(\xi)=$ $\int_{\mathbf{R}^{d}} \exp (i x \cdot \xi) \kappa(x) d x$. That is, the condition $\hat{\kappa} \in L^{\infty}\left(\mathbf{R}^{d}\right)$ is automatically verified in this case. In fact, $\hat{\kappa}$ is bounded in this case, as can be seen from $\sup _{\xi \in \mathbf{R}^{d}}|\hat{\kappa}(\xi)|=\|\kappa\|_{L^{1}\left(\mathbf{R}^{d}\right)}$.

Our proof of Theorem 5.2 proceeds in four steps; the first three are stated as lemmas.

The folklore of harmonic analysis contains precise versions of the loose assertion that "typically, kernels of positive type achieve their supremum at the origin." The first step in the proof of Theorem 5.2 is to verify a suitable form of this statement.

Lemma 5.4. Suppose $\kappa$ is a kernel of positive type such that $\hat{\kappa} \in L^{\infty}\left(\mathbf{R}^{d}\right)$. Suppose also that $\kappa$ is continuous on all of $\mathbf{R}^{d}$, and finite on $\mathbf{R}^{d} \backslash\{0\}$. Then, $\kappa(0)=\sup _{x \in \mathbf{R}^{d}} \kappa(x)$.

Proof. Recall the functions $\phi_{\epsilon}$ from the proof of Theorem 2.1; see (4.6). Then, $\kappa * \phi_{\epsilon} \in L^{1}\left(\mathbf{R}^{d}\right)$ and $\widehat{\kappa * \phi_{\epsilon}}=\hat{\kappa} \hat{\phi}_{\epsilon} \geq 0$. But ess $\sup _{\xi \in \mathbf{R}^{d}}|\hat{\kappa}(\xi)|<\infty$ and $\sup _{\xi \in \mathbf{R}^{d}}\left|\hat{\phi}_{\epsilon}(\xi)\right| \leq\left\|\phi_{\epsilon}\right\|_{L^{1}\left(\mathbf{R}^{d}\right)}=1$. Moreover, the construction of $\phi_{\epsilon}$ ensures that $\hat{\phi}_{\epsilon}$ is integrable. Therefore, $\widehat{\kappa * \phi_{\epsilon}} \in L^{1}\left(\mathbf{R}^{d}\right) \cap$ $L^{\infty}\left(\mathbf{R}^{d}\right)$, and for Lebesgue-almost all $x \in \mathbf{R}^{d}$,

$$
\left(\kappa * \phi_{\epsilon}\right)(x)=\frac{1}{(2 \pi)^{d}} \int_{\mathbf{R}^{d}} e^{-i x \cdot \xi} \hat{\kappa}(\xi) \hat{\phi}_{\epsilon}(\xi) d \xi
$$

thanks to the inversion formula for Fourier transforms. Since both sides of (5.8) are continuous functions of $x$, that equation is valid for all $x \in \mathbf{R}^{d}$. Furthermore, because $\kappa$ is a kernel of positive type, it follows that $\left(\kappa * \phi_{\epsilon}\right)(x) \leq(2 \pi)^{-d} \int_{\mathbf{R}^{d}} \hat{\kappa}(\xi) d \xi$. But if $x \neq 0$, then $\lim _{\epsilon \downarrow 0}\left(\kappa * \phi_{\epsilon}\right)(x)=\kappa(x)$, and hence,

$$
\sup _{x \in \mathbf{R}^{d} \backslash\{0\}} \kappa(x) \leq \frac{1}{(2 \pi)^{d}} \int_{\mathbf{R}^{d}} \hat{\kappa}(\xi) d \xi .
$$

It suffices to prove that

$$
\frac{1}{(2 \pi)^{d}} \int_{\mathbf{R}^{d}} \hat{\kappa}(\xi) d \xi \leq \kappa(0) .
$$

This holds trivially if $\kappa(0)$ is infinite. Therefore, we may assume without loss of generality that $\kappa(0)<\infty$. The continuity of $\kappa$ ensures that it is uniformly continuous in a neighborhood 
of the origin, thence we have $\lim _{\epsilon \downarrow 0}\left(\kappa * \phi_{\epsilon}\right)(0)=\kappa(0)$ by the classical Fejér theorem. Also, we recall that $\lim _{\epsilon \downarrow 0} \hat{\phi}_{\epsilon}(\xi)=1$ for all $\xi \in \mathbf{R}^{d}$. We use these facts in conjunction with (5.8) and Fatou's lemma to deduce (5.10), and hence the lemma.

Next we present the second step in the proof of Theorem 5.2. This is another folklore fact from harmonic analysis.

We say that a kernel $\kappa$ is lower semicontinuous if there exist a sequence of continuous functions $\kappa_{1}, \kappa_{2}, \ldots: \mathbf{R}^{d} \rightarrow \mathbf{R}_{+}$such that $\kappa_{n}(x) \leq \kappa_{n+1}(x)$ for all $n \geq 1$ and $x \in \mathbf{R}^{d}$, such that $\kappa_{n}(x) \uparrow \kappa(x)$ for all $x \in \mathbf{R}^{d}$, as $n \uparrow \infty$. Because $\kappa_{n}(x)$ is assumed to be in $\mathbf{R}_{+}$ [and not $\overline{\mathbf{R}}_{+}$], our definition of lower semicontinuity is slightly different from the usual one. Nonetheless, the following is a consequence of Lemma 12.11 of Mattila (1995, p. 161).

Lemma 5.5. Suppose $\kappa$ is a lower semicontinuous kernel of positive type on $\mathbf{R}^{d}$. Then, for all Borel probability measures $\mu$ on $\mathbf{R}^{d}$,

$$
\mathscr{E}_{\kappa}(\mu) \leq \frac{1}{(2 \pi)^{d}} \int_{\mathbf{R}^{d}} \hat{\kappa}(\xi)|\hat{\mu}(\xi)|^{2} d \xi
$$

Our next lemma constitutes the third step of our proof of Theorem 5.2.

Lemma 5.6. Suppose $\kappa$ is a continuous kernel of positive type on $\mathbf{R}^{d}$, which satisfies one of the following two conditions:

(1) $\kappa(x)<\infty$ if and only if $x \neq 0$;

(2) $\hat{\kappa} \in L^{\infty}\left(\mathbf{R}^{d}\right)$, and $\kappa(x)<\infty$ when $x \neq 0$.

Then, for all Borel probability measures $\mu$ on $\mathbf{R}^{d}$,

$$
\mathscr{E}_{\kappa}(\mu)=\frac{1}{(2 \pi)^{d}} \int_{\mathbf{R}^{d}} \hat{\kappa}(\xi)|\hat{\mu}(\xi)|^{2} d \xi .
$$

A weaker version of this result is stated in Kahane (1985, p. 134) without proof.

According to Kahane (loc. cit.), functions $\kappa$ that satisfy the conditions of Lemma 5.6 are called potential kernels. They can be defined, in equivalent terms, as kernels of positive types that are continuous on $\mathbf{R}^{d} \backslash\{0\}$ and $\lim _{x \rightarrow 0} \kappa(x)=\infty$. We will not use this terminology: the term "potential kernel" is reserved for another object.

Proof of Lemma 5.6. Regardless of whether $\kappa$ satisfies condition (1) or (2), it is lower semicontinuous. Therefore, in light of Lemma 5.5, it suffices to prove that

$$
\mathscr{E}_{\kappa}(\mu) \geq \frac{1}{(2 \pi)^{d}} \int_{\mathbf{R}^{d}} \hat{\kappa}(\xi)|\hat{\mu}(\xi)|^{2} d \xi
$$

From here on, our proof considers two separate cases:

Case 1. First, let us suppose $\kappa$ satisfies condition (1) of the lemma. 
Without loss of generality, we may assume that $\mathscr{E}_{\kappa}(\mu)<\infty$. Since $\lim _{x \rightarrow 0} \kappa(x)=\infty$, this implies that $\mu$ does not charge singletons.

According the Lusin's theorem, for every $\eta \in(0,1)$ we can find a compact set $K_{\eta} \subseteq \mathbf{R}^{d} \backslash\{0\}$ with $\mu\left(K_{\eta}^{c}\right) \leq \eta$ such that $\kappa * \mu$ is continuous - and hence uniformly continuous - on $K_{\eta}$. Define for all Borel sets $A \subseteq \mathbf{R}^{d}$,

$$
\mu_{\eta}(A)=\frac{\mu\left(A \cap K_{\eta}\right)}{1-\eta}
$$

Then $\mu_{\eta}$ is supported by the compact set $K_{\eta}$, and

$$
\frac{1}{1-\eta} \geq \mu_{\eta}\left(K_{\eta}\right)=\frac{\mu\left(K_{\eta}\right)}{1-\eta} \geq 1
$$

Now $\lim _{r \rightarrow 0} \mu_{\eta}(B(x, r))=0$ for all $x \in \mathbf{R}^{d}$, where $B(x, r)$ denotes the $\ell^{2}$-ball of radius $r>0$ about $x \in \mathbf{R}^{d}$. Therefore, a compactness argument reveals that for all $\eta \in(0,1)$,

$$
\lim _{r \downarrow 0} \sup _{x \in K_{\eta}} \mu_{\eta}(B(x, r))=0 .
$$

For otherwise we can find $\delta>0$ and $x_{r} \in K_{\eta}$ such that for all $r>0, \mu_{\eta}\left(B\left(x_{r}, r\right)\right) \geq \delta$. By compactness we can extract a subsequence $r^{\prime} \rightarrow 0$ and $x \in K_{\eta}$ such that $x_{r^{\prime}} \rightarrow x$. It follows easily then $\mu_{\eta}(B(x, \epsilon)) \geq \delta$ for all $\epsilon>0$, whence $\mu_{\eta}(\{x\}) \geq \delta$, which contradicts the fact that $\mu_{\eta}$ does not charge singletons.

Next we choose and fix $y \in K_{\eta}$ and $\eta>0$. We claim that

$$
\sup _{x \in K_{\eta}} \int_{B(x, \epsilon)} \kappa(y-z) \mu_{\eta}(d z) \rightarrow 0 \quad \text { as } \epsilon \downarrow 0 .
$$

Indeed, by (5.16) and the fact that $\left(\kappa * \mu_{\eta}\right)(y)<\infty$, we see that for all $\rho>0$ there exists $\theta>0$ such that

$$
\lim _{\epsilon \downarrow 0} \sup _{x \in K_{\eta}:|x-y| \leq \theta / 2} \int_{B(x, \epsilon)} \kappa(y-z) \mu_{\eta}(d z) \leq \rho .
$$

On the other hand, by the continuity of $\kappa$ on $\mathbf{R}^{d} \backslash\{0\}$ and (5.16), we have

$$
\lim _{\epsilon \downarrow 0} \sup _{x \in K_{\eta}:|x-y|>\theta / 2} \int_{B(x, \epsilon)} \kappa(y-z) \mu_{\eta}(d z)=0 .
$$

By combining (5.18) and (5.19), we find that

$$
\lim _{\epsilon \downarrow 0} \sup _{x \in K_{\eta}} \int_{B(x, \epsilon)} \kappa(y-z) \mu_{\eta}(d z) \leq \rho .
$$


Thus (5.17) follows from (5.20), because we can choose $\rho$ as small as we want. By (5.17) and another appeal to compactness, we obtain

$$
\sup _{y \in K_{\eta}} \sup _{x \in K_{\eta}} \int_{B(x, \epsilon)} \kappa(y-z) \mu_{\eta}(d z) \rightarrow 0 \quad \text { as } \epsilon \downarrow 0 .
$$

Consequently, for all $k \geq 1$, we can find $\epsilon_{k} \rightarrow 0$ such that

$$
\sup _{y \in K_{\eta}} \sup _{x \in K_{\eta}}\left|\left(\kappa * \mu_{\eta}\right)(y)-\int_{B\left(x, \epsilon_{k}\right)^{c}} \kappa(y-z) \mu_{\eta}(d z)\right| \leq \frac{1}{k} .
$$

Let $y \in K_{\eta}$ and let $\left\{y_{n}\right\}_{n=1}^{\infty}$ be an arbitrary sequence in $K_{\eta}$ such that $\lim _{n \rightarrow \infty} y_{n}=y$. Because $z \mapsto \kappa(y-z)$ is uniformly continuous on $B\left(y, \epsilon_{k}\right)^{c} \cap K_{\eta}$, we have

$$
\lim _{n \rightarrow \infty} \int_{B\left(y, \epsilon_{k}\right)^{c}} \kappa\left(y_{n}-z\right) \mu_{\eta}(d z)=\int_{B\left(y, \epsilon_{k}\right)^{c}} \kappa(y-z) \mu_{\eta}(d z) .
$$

This and (5.22) together imply that for all $k \geq 1$,

$$
\lim _{n \rightarrow \infty}\left|\left(\kappa * \mu_{\eta}\right)\left(y_{n}\right)-\left(\kappa * \mu_{\eta}\right)(y)\right| \leq \frac{2}{k} .
$$

Let $k \uparrow \infty$ to deduce that $\kappa * \mu_{\eta}$ is continuous, and hence uniformly continuous, on $K_{\eta}$. On the other hand, it can be verified directly that $\kappa * \mu_{\eta}$ is continuous on $K_{\eta}^{c}$. Hence, we have shown that $\kappa * \mu_{\eta}$ is continuous on $\mathbf{R}^{d}$.

If $0<\epsilon, \eta<1$, then we can appeal to the Fubini-Tonelli theorem, a few times in succession, to deduce that

$$
\mathscr{E}_{\kappa * \psi_{\epsilon}}\left(\mu_{\eta}\right)=\int \kappa(-z)\left(a_{\epsilon, \eta} * b_{\epsilon, \eta}\right)(z) d z
$$

where $a_{\epsilon, \eta}:=\phi_{\epsilon} * \mu_{\eta}, b_{\epsilon, \eta}:=\phi_{\epsilon} * \breve{\mu}_{\eta}$, and $\breve{\mu}_{\eta}$ is the Borel probability measure on $\mathbf{R}^{d}$ that is defined by

$$
\breve{\mu}_{\eta}(A):=\mu_{\eta}(-A) \quad \text { for all } A \subseteq \mathbf{R}^{d} .
$$

We observe the following elementary facts:

(1) Both $a_{\epsilon, \eta}$ and $b_{\epsilon, \eta}$ are infinitely differentiable functions of compact support;

(2) because $\phi_{\epsilon}$ is of positive type, the Fourier transform of $a_{\epsilon, \eta} * b_{\epsilon, \eta}$ is $\left|\hat{\phi}_{\epsilon}\right|^{2}\left|\hat{\mu}_{\eta}\right|^{2}$;

(3) the Fourier transform of $z \mapsto \kappa(-z)$ is the same as that of $\kappa$ because $\kappa$ is of positive type;

Therefore, we can combine the preceding with the Parseval identity, and deduce that

$$
\begin{aligned}
\mathscr{E}_{\kappa * \psi_{\epsilon}}\left(\mu_{\eta}\right) & =\frac{1}{(2 \pi)^{d}} \int_{\mathbf{R}^{d}} \hat{\kappa}(\xi) \overline{\hat{a}_{\epsilon, \eta}(\xi) \hat{b}_{\epsilon, \eta}(\xi)} d \xi \\
& =\frac{1}{(2 \pi)^{d}} \int_{\mathbf{R}^{d}} \hat{\kappa}(\xi)\left|\hat{\phi}_{\epsilon}(\xi)\right|^{2}\left|\hat{\mu}_{\eta}(\xi)\right|^{2} d \xi .
\end{aligned}
$$


We can apply the Fubini-Tonelli theorem to write the left-most term in another way, as well. Namely,

$$
\mathscr{E}_{\kappa * \psi_{\epsilon}}\left(\mu_{\eta}\right)=\int\left(\kappa * \psi_{\epsilon} * \mu_{\eta}\right) d \mu_{\eta}
$$

The continuity of $\kappa * \mu_{\eta}$, and Fejér's theorem, together imply that $\kappa * \mu_{\eta} * \psi_{\epsilon}$ converges to $\kappa * \mu_{\eta}$ uniformly on $K_{\eta}$ as $\epsilon \downarrow 0$, and hence $\mathscr{E}_{\kappa * \psi_{\epsilon}}\left(\mu_{\eta}\right)$ converges to $\mathscr{E}_{\kappa}\left(\mu_{\eta}\right)$ as $\epsilon \downarrow 0$. This and (5.27) together imply that

$$
\begin{aligned}
\mathscr{E}_{\kappa}\left(\mu_{\eta}\right) & =\frac{1}{(2 \pi)^{d}} \lim _{\epsilon \rightarrow 0} \int_{\mathbf{R}^{d}} \hat{\kappa}(\xi)\left|\hat{\phi}_{\epsilon}(\xi)\right|^{2}\left|\hat{\mu}_{\eta}(\xi)\right|^{2} d \xi \\
& \geq \frac{1}{(2 \pi)^{d}} \int_{\mathbf{R}^{d}} \hat{\kappa}(\xi)\left|\hat{\mu}_{\eta}(\xi)\right|^{2} d \xi,
\end{aligned}
$$

owing to Fatou's lemma. Since $\mu_{\eta}$ is $(1-\eta)^{-1}$ times a restriction of $\mu$, it follows that

$$
\frac{1}{(1-\eta)^{2}} \mathscr{E}_{\kappa}(\mu) \geq \frac{1}{(2 \pi)^{d}} \int_{\mathbf{R}^{d}} \hat{\kappa}(\xi)\left|\hat{\mu}_{\eta}(\xi)\right|^{2} d \xi
$$

Because for all $\xi \in \mathbf{R}^{d}$,

$$
\left|\hat{\mu}_{\eta}(\xi)-\frac{\hat{\mu}(\xi)}{1-\eta}\right| \leq \frac{\mu\left(K_{\eta}^{c}\right)}{1-\eta} \leq \frac{\eta}{1-\eta},
$$

we deduce that $\lim _{\eta \rightarrow 0} \hat{\mu}_{\eta}=\hat{\mu}$ pointwise. An appeal to Fatou's lemma justifies (5.13), and this completes our proof in the first case.

Case 2. Next, we consider the case that $\kappa$ satisfies condition (2) of the lemma. If $\kappa(0)=\infty$, then condition (1) is satisfied, and therefore the proof is complete. Thus, we may assume that $\kappa(0)<\infty$. According to Lemma 5.4, $\kappa$ is a bounded and continuous function from $\mathbf{R}^{d}$ into $\mathbf{R}_{+}$. For all Borel sets $A \subseteq \mathbf{R}^{d}$ define

$$
\mu_{n}(A):=\frac{\mu_{n}\left(A \cap[-n, n]^{d}\right)}{\chi_{n}}, \quad \text { where } \quad \chi_{n}:=\mu\left([-n, n]^{d}\right) .
$$

If $n>0$ is sufficiently large, then $\mu_{n}$ is a well-defined Borel probability measure on $[-n, n]^{d}$, and since $\kappa$ is uniformly continuous on $[-n, n]^{d}$,

$$
\begin{aligned}
\frac{1}{\chi_{n}^{2}} \mathscr{E}_{\kappa}(\mu) & \geq \mathscr{E}_{\kappa}\left(\mu_{n}\right) \\
& =\lim _{\epsilon \downarrow 0} \mathscr{E}_{\kappa * \psi_{\epsilon}}\left(\mu_{n}\right) \\
& =\frac{1}{(2 \pi)^{d}} \lim _{\epsilon \downarrow 0} \int_{\mathbf{R}^{d}} \hat{\kappa}(\xi) \hat{\psi}_{\epsilon}(\xi)\left|\hat{\mu}_{n}(\xi)\right|^{2} d \xi .
\end{aligned}
$$


The first equality holds because $\kappa * \psi_{\epsilon}$ converges uniformly to $\kappa$ on $[-n, n]^{d}$, as $\epsilon \downarrow 0$. The second is a consequence of the Parseval identity. Thanks to Fatou's lemma, we have proved that for all $n>0$ sufficiently large,

$$
\frac{1}{\chi_{n}^{2}} \mathscr{E}_{\kappa}(\mu) \geq \frac{1}{(2 \pi)^{d}} \int_{\mathbf{R}^{d}} \hat{\kappa}(\xi)\left|\hat{\mu}_{n}(\xi)\right|^{2} d \xi
$$

As $n$ tends to infinity, $\chi_{n} \uparrow 1$ and $\hat{\mu}_{n} \rightarrow \hat{\mu}$ pointwise. Therefore, another appeal to Fatou's lemma implies (5.13), and hence the lemma.

Now we derive Theorem 5.2 .

Proof of Theorem 5.2. Let us observe that by the Fubini-Tonelli theorem,

$$
\mathscr{E}_{\kappa * \nu}(\mu)=\mathscr{E}_{\kappa}(\mu, \breve{\nu} * \mu) .
$$

In fact, both sides are equal to $\mathrm{E}\left[\kappa\left(X-X^{\prime}-Y\right)\right]$, where $\left(X, X^{\prime}, Y\right)$ are independent, $X$ and $X^{\prime}$ are distributed as $\mu$, and $Y$ is distributed as $\nu$.

Lemma 5.6, and polarization, together imply that the following holds for every Borel probability measure $\sigma$ on $\mathbf{R}^{d}$ :

$$
\mathscr{E}_{\kappa}(\mu, \sigma)=\frac{1}{(2 \pi)^{d}} \int_{\mathbf{R}^{d}} \hat{\kappa}(\xi) \hat{\sigma}(\xi) \overline{\hat{\mu}(\xi)} d \xi .
$$

Indeed, we first notice that

$$
\mathscr{E}_{\kappa}\left(\frac{\mu+\sigma}{2}\right)=\frac{1}{4} \mathscr{E}_{\kappa}(\mu)+\frac{1}{4} \mathscr{E}_{\kappa}(\sigma)+\frac{1}{2} \mathscr{E}_{\kappa}(\mu, \sigma) .
$$

Thus, we solve for $\mathscr{E}_{\kappa}(\mu, \sigma)$ and apply Lemma 5.6 to deduce that

$$
\begin{aligned}
\mathscr{E}_{\kappa}(\mu, \sigma)=\frac{2}{(2 \pi)^{d}} \int_{\mathbf{R}^{d}} \hat{\kappa}(\xi) \mid & \mid \widehat{\left.\left(\frac{\mu+\sigma}{2}\right)(\xi)\right|^{2} d \xi} \\
& -\frac{1}{2(2 \pi)^{d}} \int_{\mathbf{R}^{d}} \hat{\kappa}(\xi)|\hat{\mu}(\xi)|^{2} d \xi-\frac{1}{2(2 \pi)^{d}} \int_{\mathbf{R}^{d}} \hat{\kappa}(\xi)|\hat{\sigma}(\xi)|^{2} d \xi .
\end{aligned}
$$

We solve to obtain (5.36).

Thus we define $\sigma:=\breve{\nu} * \mu$, observe that $\sigma$ is a Borel probability measure on $\mathbf{R}^{d}$, and $\mathscr{E}_{\kappa * \nu}(\mu)=\mathscr{E}_{\kappa}(\mu, \sigma)$. Thus, we may apply (5.35) and (5.36) - in this order - to find that

$$
\begin{aligned}
\mathscr{E}_{\kappa * \nu}(\mu) & =\frac{1}{(2 \pi)^{d}} \int_{\mathbf{R}^{d}} \hat{\kappa}(\xi) \hat{\sigma}(\xi) \overline{\hat{\mu}(\xi)} d \xi \\
& =\frac{1}{(2 \pi)^{d}} \int_{\mathbf{R}^{d}} \hat{\kappa}(\xi) \hat{\nu}(-\xi)|\hat{\mu}(\xi)|^{2} d \xi .
\end{aligned}
$$


In the last line, we have only used the fact that $\hat{\sigma}(\xi)=\hat{\nu}(-\xi) \hat{\mu}(\xi)$ for all $\xi \in \mathbf{R}^{d}$. Because the left-most term in (5.39) is real-valued, so is the right-most term. Therefore, we may consider only the real part of the right-most item in (5.39), and this proves the result.

\section{Absolute continuity considerations}

Suppose $E$ is a Borel measurable subset of $\mathbf{R}^{N}$ that has positive Lebesgue measure, and $Y:=\{Y(\boldsymbol{t})\}_{\boldsymbol{t} \in E}$ is an $\mathbf{R}^{d}$-valued random field that is indexed by $E$.

Definition 6.1. We say that $Y$ has a one-potential density $v$ if $v \in L^{1}\left(\mathbf{R}^{d}\right)$ is nonnegative and satisfies the following for all Borel measurable functions $f: \mathbf{R}^{d} \rightarrow \mathbf{R}_{+}$:

$$
\frac{1}{\int_{E} e^{-[\boldsymbol{s}]} d \boldsymbol{s}} \mathrm{E}\left[\int_{E} f(Y(\boldsymbol{t})) e^{-[\boldsymbol{t}]} d \boldsymbol{t}\right]=\int_{\mathbf{R}^{d}} f(x) v(x) d x .
$$

In particular, $\widetilde{\mathfrak{X}}$ has a one-potential density $v$ if for all Borel measurable functions $f$ : $\mathbf{R}^{d} \rightarrow \mathbf{R}_{+}$,

$$
(R f)(x)=\int_{\mathbf{R}^{d}} f(x+y) v(y) d y:=(f * \breve{v})(x) \quad \text { for all } x \in \mathbf{R}^{d},
$$

where $\breve{g}(z):=g(-z)$ for all functions $g$. It follows from Lemma 2.1 in Hawkes (1979) that $\widetilde{\mathfrak{X}}$ has a one-potential density if and only if the operator $R$ defined by (2.9) is strong Feller. That is, if $R f$ is continuous whenever $f$ is Borel measurable and has compact support.

In order to be concrete, we choose a "nice" version of the one-potential density $v$, when it exists. Before we proceed further, let us observe that when $v$ exists it is a probability density. In particular, the Lebesgue density theorem tell us that

$$
v(x)=\lim _{\epsilon \downarrow 0} \frac{1}{\lambda_{d}(B(0, \epsilon))} \int_{B(x, \epsilon)} v(y) d y \quad \text { for almost all } x \in \mathbf{R}^{d}\left[\lambda_{d}\right] .
$$

We can recognize the integral as $\left(R \mathbf{1}_{B(0, \epsilon)}\right)(-x)$. Moreover, we can alter $v$ on a Lebesgue-null set and still obtain a one-potential density for $\widetilde{\mathfrak{X}}$. Therefore, from now on, we always choose the following version of $v$ :

$$
v(x):=\liminf _{\epsilon \downarrow 0} \frac{\left(R \mathbf{1}_{(0, \epsilon)}\right)(-x)}{\lambda_{d}(B(0, \epsilon))} \quad \text { for all } x \in \mathbf{R}^{d} .
$$

Lemma 2.3 states that the Fourier multiplier of $R$ is $K_{\Psi}$. Therefore, the Fourier transform of $\breve{v}$ is also $K_{\Psi}$; confer with (6.2). Because $K_{\Psi}$ is real-valued, this proves the following:

Lemma 6.2. If $\widetilde{\mathfrak{X}}$ has a one-potential density $v$, then $\hat{v}=K_{\Psi}$ in the sense of Schwartz. In particular, $v$ is an integrable kernel of positive type on $\mathbf{R}^{d}$.

Remark 6.3. Let $\epsilon_{1}, \ldots, \epsilon_{N}$ denote $N$ random variables, all independent of one another, as well as $\left\{X_{j}\right\}_{j=1}^{N}$ and $\left\{X_{j}^{\prime}\right\}_{j=1}^{N}$, with $\mathrm{P}\left\{\epsilon_{1}= \pm 1\right\}=1 / 2$. We can re-organize the order of 
integration a few times to find that

$$
\frac{1}{2^{N}} \mathrm{E}\left[\int_{\mathbf{R}^{N}} f(\widetilde{\mathfrak{X}}(\boldsymbol{t})) e^{-[\boldsymbol{t}]} d \boldsymbol{t}\right]=\mathrm{E}\left[\int_{\mathbf{R}_{+}^{N}} f\left(\sum_{j=1}^{N} \epsilon_{j} X_{j}\left(s_{j}\right)\right) e^{-[\boldsymbol{s}]} d \boldsymbol{s}\right] .
$$

Thus, in particular, $\widetilde{\mathfrak{X}}$ has a one-potential density if and only if the $\mathbf{R}_{+}^{N}$-indexed random field $\left(t_{1}, \ldots, t_{N}\right) \mapsto \sum_{j=1}^{N} \epsilon_{j} X_{j}\left(t_{j}\right)$ does [interpreted in the obvious sense]. Interestingly enough, the latter random field appears earlier - though for quite different reasons as ours - in the works of Marcus and Rosen (1999b, 1999a).

Remark 6.4. It follows from the definitions that a random field $\{Y(\boldsymbol{t})\}_{\boldsymbol{t} \in E}$ has a one-potential density if and only if the Borel probability measure

$$
\mathbf{R}^{d} \supseteq A \mapsto \mathrm{E}\left[\int_{E} \mathbf{1}_{A}(Y(\boldsymbol{t})) e^{-[\boldsymbol{t}]} d \boldsymbol{t}\right] \quad \text { is absolutely continuous. }
$$

Now suppose $f \geq 0$ is Borel measurable. Then

$$
\int_{\mathbf{R}^{N}} f(\widetilde{\mathfrak{X}}(\boldsymbol{t})) e^{-[\boldsymbol{t}]} d \boldsymbol{t} \geq \int_{\mathbf{R}_{+}^{N}} f(\mathfrak{X}(\boldsymbol{t})) e^{-[\boldsymbol{t}]} d \boldsymbol{t} .
$$

We take expectations of both sides to deduce from (6.6) that if $\widetilde{\mathfrak{X}}$ has a one-potential density, then so does $\mathfrak{X}$. When $N=1$, it can be verified that the converse it also true. However, the previous remark can be used to show that, when $N \geq 2$, the converse is not necessarily true. Thus, we can conclude that the existence of a one-potential density for $\widetilde{\mathfrak{X}}$ is a more stringent condition than the existence of a one-potential density for $\mathfrak{X}$. Another consequence of the preceding is the following: If the one-potential density of $\widetilde{\mathfrak{X}}$ exists and the potential density of $\mathfrak{X}$ is a.e.-positive, then the one-potential density of $\widetilde{\mathfrak{X}}$ is per force also a.e.-positive. We have, and will, encounter these conditions several times.

Our next theorem is the main result of this subsection. From a technical point of view, it is also a key result in this paper. In order to describe it properly, we introduce some notation first.

If $T$ is a nonempty subset of $\{1, \ldots, N\}$, then we define $|T|$ to be the cardinality of $T$, and $\mathfrak{X}_{T}$ to be the subprocess associated to the index $T$. That is, $\mathfrak{X}_{T}$ is the following $|T|$-parameter, $\mathbf{R}^{d}$-valued random field:

$$
\mathfrak{X}_{T}(\boldsymbol{t}):=\sum_{j \in T} X_{j}\left(t_{j}\right) \quad \text { for all } \boldsymbol{t} \in \mathbf{R}_{+}^{|T|} .
$$

In order to obtain nice formulas, we define $\mathfrak{X}_{\varnothing}$ to be the constant 0 . In this way, it follows that, regardless of whether or not $|T|>0$, each $\mathfrak{X}_{T}$ is itself an additive Lévy process, and the Lévy exponent of $\mathfrak{X}_{T}$ is the function $\left(\Psi_{j}\right)_{j \in T}: \mathbf{R}^{d} \rightarrow \mathbf{C}^{|T|}$. Thus, we can talk about the 
stationary field $\widetilde{\mathfrak{X}}_{T}$ for all $T \subseteq\{1, \ldots, N\}$, etc. Despite this new notation, we continue to write $\mathfrak{X}$ and $\widetilde{\mathfrak{X}}$ in place of the more cumbersome $\mathfrak{X}_{\{1, \ldots, N\}}$ and $\widetilde{\mathfrak{X}}_{\{1, \ldots, N\}}$.

Theorem 6.5. Suppose there exists a nonrandom and nonempty subset $T \subseteq\{1, \ldots, N\}$, such that $\widetilde{\mathfrak{X}}_{T}$ has a one-potential density $v_{T}: \mathbf{R}^{d} \mapsto \overline{\mathbf{R}}_{+}$that is continuous on $\mathbf{R}^{d}$, and finite on $\mathbf{R}^{d} \backslash\{0\}$. Then, $\widetilde{\mathfrak{X}}$ has a one-potential density $v$ on $\mathbf{R}^{d}$, and for all Borel sets $F \subseteq \mathbf{R}^{d}$,

$$
\mathrm{E}\left[\lambda_{d}\left(\mathfrak{X}\left(\mathbf{R}_{+}^{N}\right) \oplus F\right)\right]>0 \quad \Leftrightarrow \quad \mathscr{C}_{v}(F)>0
$$

Proof. First, consider the case that $T=\{1, \ldots, N\}$. In this case, $\widetilde{\mathfrak{X}}$ has a one-potential density $v:=v_{\{1, \ldots, N\}}$ that is continuous on $\mathbf{R}^{d}$, and finite away from the origin. In light of Remark 5.3, Lemma 6.2 shows us that $\kappa:=v$ satisfies condition (2) of Theorem 5.2, Theorem 5.2, in turn, implies that $I_{\Psi}(\mu)=\mathscr{E}_{v}(\mu)$ for all Borel probability measures $\mu$ on $\mathbf{R}^{d}$, and thence $\mathscr{C}_{v}(F)=\operatorname{cap}_{\boldsymbol{\Psi}}(F)$. This and Theorem [1.1 together imply Theorem 6.5] in the case that $\widetilde{\mathfrak{X}}$ has a continuous one-potential density that is finite on $\mathbf{R}^{d} \backslash\{0\}$.

Next, consider the remaining case that $1 \leq|T| \leq N-1$. For all Borel sets $A \subseteq \mathbf{R}^{d}$ define

$$
V_{T^{c}}(A):=\mathrm{E}\left[\int_{\mathbf{R}^{N-|T|}} \mathbf{1}_{A}\left(\widetilde{\mathfrak{X}}_{T^{c}}(\boldsymbol{s})\right) e^{-[\boldsymbol{s}]} d \boldsymbol{s}\right] .
$$

This is the one-potential measure for the stationary field based on the additive Lévy process $\mathfrak{X}_{T^{c}}$. The corresponding object for $\mathfrak{X}_{T}$ can be defined likewise, viz.,

$$
V_{T}(A):=\mathrm{E}\left[\int_{\mathbf{R}^{|T|}} \mathbf{1}_{A}\left(\widetilde{\mathfrak{X}}_{T}(\boldsymbol{t})\right) e^{-[\boldsymbol{t}]} d \boldsymbol{t}\right] .
$$

We can observe that

$$
\begin{aligned}
V_{T^{c}}(A) & :=\mathrm{E}\left[\int_{\mathbf{R}_{+}^{N}} \mathbf{1}_{A}\left(\sum_{j \in T^{c}} \epsilon_{j} X_{j}\left(s_{j}\right)\right) e^{-[\boldsymbol{s}]} d \boldsymbol{s}\right], \\
V_{T}(A) & :=\mathrm{E}\left[\int_{\mathbf{R}_{+}^{N}} \mathbf{1}_{A}\left(\sum_{j \in T} \epsilon_{j} X_{j}\left(s_{j}\right)\right) e^{-[\boldsymbol{s}]} d \boldsymbol{s}\right],
\end{aligned}
$$

where $\epsilon_{1}, \ldots, \epsilon_{N}$ are i.i.d. random functions that are totally independent of $X_{1}, \ldots, X_{N}$ and $\widetilde{X}_{1}, \ldots, \widetilde{X}_{N}$, and $\mathrm{P}\left\{\epsilon_{1}= \pm 1\right\}=1 / 2$.

Similarly, we can write for all Borel measurable functions $f: \mathbf{R}^{d} \rightarrow \mathbf{R}_{+}$and $x \in \mathbf{R}^{d}$,

$$
(R f)(x)=\mathrm{E}\left[\int_{\mathbf{R}_{+}^{N}} f\left(x+\sum_{j=1}^{N} \epsilon_{j} X_{j}\left(t_{j}\right)\right) e^{-[\boldsymbol{t}]} d \boldsymbol{t}\right] .
$$


Note that the integral is now over $\mathbf{R}_{+}^{N}$ [and not $\mathbf{R}^{N}$ ]. Moreover, we can write

$$
\begin{aligned}
(R f)(x) & =\iint f(x+y+z) V_{T}(d y) V_{T^{c}}(d z) \\
& =\int f(x+y)\left(V_{T} * V_{T^{c}}\right)(d x),
\end{aligned}
$$

The condition that $\widetilde{\mathfrak{X}}_{T}$ has a one-potential density $v_{T}$ is equivalent to the statement that $V_{T}(d y)=v_{T}(y) d y$, whence it follows that $(R f)(x)=\int f(x+y) v(y) d y$, where

$$
v(y):=\int v_{T}(y-z) V_{T^{c}}(d z)
$$

This proves the assertion that $\widetilde{\mathfrak{X}}$ has a one-potential density $v$. Note that $v$ is of the form $\kappa * \nu$, where $\kappa:=v_{T}$ and $\nu:=V_{T^{c}}$. Thus, another appeal to Theorem 5.2 shows that condition (2) there is satisfied [confer also with Remark 5.3], and thence it follows that $\mathscr{C}_{v}(F)=\operatorname{cap}_{\boldsymbol{\Psi}}(F)$. This and Theorem 1.1 together complete the proof.

6.1. A relation to an intersection problem. Define for all Borel sets $F \subseteq \mathbf{R}^{d}$,

$$
\begin{aligned}
& \mathrm{H}_{F}:=\left\{x \in \mathbf{R}^{d}: \mathrm{P}\left\{\mathfrak{X}\left((0, \infty)^{N}\right) \cap(x \oplus F) \neq \varnothing\right\}>0\right\} \\
& \widetilde{\mathrm{H}}_{F}:=\left\{x \in \mathbf{R}^{d}: \mathrm{P}\left\{\widetilde{\mathfrak{X}}\left(\mathbf{R}_{\neq}^{N}\right) \cap(x \oplus F) \neq \varnothing\right\}>0\right\},
\end{aligned}
$$

where $\mathbf{R}_{\neq}^{N}:=\left\{\boldsymbol{t} \in \mathbf{R}^{N}: t_{j} \neq 0\right.$ for every $\left.j=1, \ldots, N\right\}$. Clearly, $\mathrm{H}_{F} \subseteq \widetilde{\mathrm{H}}_{F}$.

The following improves on Proposition 6.2 of Khoshnevisan, Xiao, and Zhong (2003).

Proposition 6.6. Consider the following statements:

(0) $\mathrm{E}\left[\lambda_{d}\left(\mathfrak{X}\left(\mathbf{R}_{+}^{N}\right) \oplus F\right)\right]>0$;

(1) $\operatorname{cap}_{\Psi}(F)>0$;

(2) $\mathscr{C}_{v}(F)>0$;

(3) $\lambda_{d}\left(\mathrm{H}_{F}\right)>0$;

(4) $\mathrm{H}_{F} \neq \varnothing$;

(5) $\widetilde{\mathrm{H}}_{F}=\mathbf{R}^{d}$; and

(6) $\mathrm{H}_{F}=\mathbf{R}^{d}$.

Then:

(a) It is always the case that $(0) \Leftrightarrow(1)$.

(b) If $\mathfrak{X}$ has a one-potential density, then $(1) \Leftrightarrow(3) \Leftrightarrow(4)$.

(c) If $\mathfrak{X}$ has an a.e.-positive one-potential density, then $(1) \Leftrightarrow(3) \Leftrightarrow(4) \Leftrightarrow(6)$.

(d) If there exists a non-empty $T \subseteq\{1, \ldots, N\}$ such that the sub-process $\widetilde{\mathfrak{X}}_{T}$ has a onepotential density $v_{T}$, and $v_{T}$ is continuous on $\mathbf{R}^{d}$ and finite on $\mathbf{R}^{d} \backslash\{0\}$. Then, $(1) \Leftrightarrow(2) \Leftrightarrow(3) \Leftrightarrow(4)$. 
(e) Suppose the conditions of (e) are met, and let $v$ denote the one-potential density of $\widetilde{\mathfrak{X}}$. If $v>0$ a.e., then $(1) \Leftrightarrow(2) \Leftrightarrow(3) \Leftrightarrow(4) \Leftrightarrow(5)$.

Remark 6.7. Proposition 6.6 is deceptively subtle. For example, The preceding proposition might fail to hold if $\mathrm{H}_{F}$ were replaced by the related set

$$
\mathrm{H}_{F}^{*}:=\left\{x \in \mathbf{R}^{d}: \mathrm{P}\left\{\mathfrak{X}\left(\mathbf{R}_{+}^{N}\right) \cap(x \oplus F) \neq \varnothing\right\}>0\right\} .
$$

Indeed, because $\mathfrak{X}(\mathbf{0})=0$, it follows that $\mathrm{H}_{F}^{*}=-F$.

Now we prove Proposition 6.6.

Proof of Proposition 6.6. Part (a) is merely a restatement of Theorem 1.1,

We first observe the following consequence of the Fubini-Tonelli theorem:

$$
\mathrm{E}\left[\lambda_{d}\left(\mathfrak{X}\left((0, \infty)^{N}\right) \ominus F\right)\right]=\int_{\mathbf{R}^{d}} \mathrm{P}\left\{\mathfrak{X}\left((0, \infty)^{N}\right) \cap(x \oplus F) \neq \varnothing\right\} d x .
$$

See (2.7) for a similar computation. If $\operatorname{cap}_{\Psi}(F)>0$, then the left-hand side of (6.18) is positive by a simple variant of Theorem 1.1. This proves that $(1) \Rightarrow(3)$. Conversely, if $\lambda_{d}\left(\mathrm{H}_{F}\right)>0$, then the right-hand side of (6.18) - and hence the left-hand side -are positive. Another appeal to Theorem 1.1 shows us that $(1) \Leftrightarrow(3)$.

Now we finish the proof of (b). Clearly, (3) implies (4). In order to prove that $(4) \Rightarrow(3)$, we define

$$
Q_{s}:=\left(s_{1}, \infty\right) \times \cdots \times\left(s_{N}, \infty\right) \quad \text { for all } s \in \mathbf{R}^{N} .
$$

We note that for all $s \in \mathbf{R}^{N}$ and $x \in \mathbf{R}^{d}$,

$$
\begin{aligned}
\mathrm{P}\left\{\widetilde{\mathfrak{X}}\left(Q_{\boldsymbol{s}}\right) \cap(x \oplus F)\right. & \neq \varnothing\} \\
& =\int_{\mathbf{R}^{d}} \mathrm{P}\left\{\mathfrak{X}\left((0, \infty)^{N}\right) \cap((x-y) \oplus F) \neq \varnothing\right\} \mathrm{P}\{\widetilde{\mathfrak{X}}(s) \in d y\} .
\end{aligned}
$$

This uses only the fact that $\{\widetilde{\mathfrak{X}}(\boldsymbol{t})-\widetilde{\mathfrak{X}}(\boldsymbol{s})\}_{\boldsymbol{t} \in Q_{s}}$ is independent of $\widetilde{\mathfrak{X}}(\boldsymbol{s})$, and has the same law as $\mathfrak{X}$. In particular,

$$
\begin{aligned}
\int_{\mathbf{R}^{N}} \mathrm{P}\left\{\widetilde{\mathfrak{X}}\left(Q_{\boldsymbol{s}}\right) \cap(x \oplus F)\right. & \neq \varnothing\} e^{-[\boldsymbol{s}]} d \boldsymbol{s} \\
& =2^{N} \int_{\mathbf{R}^{d}} \mathrm{P}\left\{\mathfrak{X}\left((0, \infty)^{N}\right) \cap((x-y) \oplus F) \neq \varnothing\right\} v(y) d y,
\end{aligned}
$$

where $v$ denotes the one-potential density of $\widetilde{\mathfrak{X}}$. [It exists thanks to Remark 6.4],

If (4) holds, then there exists $s \in \mathbf{R}_{+}^{N}$ such that $\mathrm{P}\left\{\mathfrak{X}\left(Q_{\boldsymbol{s}}\right) \cap(x \oplus F) \neq \varnothing\right\}>0$. Consequently, $\mathrm{P}\left\{\widetilde{\mathfrak{X}}\left(Q_{\boldsymbol{s}}\right) \cap(x \oplus F) \neq \varnothing\right\}>0$. As we decrease $\boldsymbol{s}$, coordinatewise, the preceding probability increases. Therefore, the left-most term in (6.21) is (strictly) positive, and therefore 
so is the right-most term in (6.21). We can conclude that $\mathrm{P}\left\{\mathfrak{X}\left((0, \infty)^{N}\right) \cap(w \oplus F) \neq \varnothing\right\}>0$ for all $w$ in a non-null set $\left[\lambda_{d}\right]$. This proves $(3) \Rightarrow(4)$, whence (b).

In order to prove (c), let $g$ denote the one-potential density of $\mathfrak{X}$. Then, we argue as we did to prove (6.21), and deduce that

$$
\begin{aligned}
\int_{\mathbf{R}_{+}^{N}} \mathrm{P}\left\{\mathfrak{X}\left(Q_{\boldsymbol{s}}\right) \cap(x \oplus F) \neq \varnothing\right\} e^{-[\boldsymbol{s}]} d \boldsymbol{s} \\
\quad=\int_{\mathbf{R}^{d}} \mathrm{P}\left\{\mathfrak{X}\left((0, \infty)^{N}\right) \cap((x-y) \oplus F) \neq \varnothing\right\} g(y) d y .
\end{aligned}
$$

If $\mathrm{H}_{F} \neq \mathbf{R}^{d}$, then the left-most term must be zero for some $x \in \mathbf{R}^{d}$. Because $g>0$ a.e., this proves that $\lambda_{d}\left(\mathrm{H}_{F}\right)=0$.

The equivalence of $(1) \Leftrightarrow(2)$ in $(d)$ is contained in Theorems 1.1 and 6.5. The rest of Part (d) is proved similarly as in the proof of (b).

To prove (e): We note that " $\widetilde{H}_{F}=\mathbf{R}^{d}$ iff (4)" [under the condition that $v>0$ a.e.] has a very similar proof to " $\mathrm{H}_{F}=\mathbf{R}^{d}$ iff (4)" [under the condition that $g>0$ a.e.], but uses (6.21) instead of (6.22). We omit the details and conclude our proof.

\section{Proof of Theorem 1.3}

We continue to let $X_{1}, \ldots, X_{N}$ denote $N$ independent Lévy processes on $\mathbf{R}^{d}$ with respective exponents $\Psi_{1}, \ldots, \Psi_{N}$. There is a large literature that is devoted to the " $N$-parameter Lévy process" $\otimes_{j=1}^{N} X_{j}$ defined by

$$
\left(\otimes_{j=1}^{N} X_{j}\right)(\boldsymbol{t}):=\left(\begin{array}{c}
X_{1}\left(t_{1}\right) \\
\vdots \\
X_{N}\left(t_{N}\right)
\end{array}\right) \quad \text { for all } \boldsymbol{t} \in \mathbf{R}_{+}^{N} .
$$

Note that:

(i) The state space of $\otimes_{j=1}^{N} X_{j}$ is $\left(\mathbf{R}^{d}\right)^{N}$; and

(ii) In the special case that $N=2$, (7.1) reduces to (1.2).

In this section we describe how this theory, and much more, is contained within the theory of additive Lévy processes of this paper.

Let us begin by making the observation that product Lévy processes are in fact degenerate additive Lévy processes. Indeed, for all $\boldsymbol{t} \in \mathbf{R}_{+}^{N}$ we can write

$$
\left(\otimes_{j=1}^{N} X_{j}\right)(\boldsymbol{t})=\boldsymbol{A}^{1} X_{1}\left(t_{1}\right)+\cdots+\boldsymbol{A}^{N} X_{N}\left(t_{N}\right),
$$


where each $X_{j}\left(t_{j}\right)$ is viewed as a column vector with dimension $d$, and each $\boldsymbol{A}^{j}$ is the $N d \times d$ matrix

$$
\boldsymbol{A}^{j}=\left(\begin{array}{c}
\mathbf{0}_{(j-1) d \times d} \\
\boldsymbol{I}_{d \times d} \\
\mathbf{0}_{(N-j) d \times d}
\end{array}\right)
$$

Here: (i) $\mathbf{0}_{(j-1) d \times d}$ is a $(j-1) d \times d$ matrix of zeros; and (ii) $\boldsymbol{I}_{d \times d}$ is the identity matrix with $d^{2}$ entries. We emphasize that the matrix $\boldsymbol{I}_{d \times d}$ appears after $j-1$ square matrices of size $d^{2}$ whose entries are all zeroes.

It is also easy to describe the law of the process $\otimes_{j=1}^{N} X_{j}$ via the following characteristicfunction relation:

$$
\mathrm{E}\left[\exp \left\{i \sum_{j=1}^{N} \xi^{j} \cdot X_{j}\left(t_{j}\right)\right\}\right]=\exp \left(-\sum_{j=1}^{N} t_{j} \Psi_{j}\left(\xi^{j}\right)\right),
$$

valid for all $\xi^{1}, \ldots, \xi^{N} \in \mathbf{R}^{d}$ and $t_{1}, \ldots, t_{N} \geq 0$. Therefore, the exponent of the $N$-parameter additive Lévy process $\otimes_{j=1}^{N} X_{j}$ is

$$
\boldsymbol{\Psi}\left(\xi^{1}, \ldots, \xi^{N}\right)=\left(\begin{array}{c}
\Psi_{1}\left(\xi^{1}\right) \\
\vdots \\
\Psi_{N}\left(\xi^{N}\right)
\end{array}\right) \quad \text { for all } \quad \xi^{1}, \ldots, \xi^{N} \in \mathbf{R}^{d}
$$

Therefore, Theorem 1.1 applies, without further thought, to yield the following.

Corollary 7.1. Choose and fix a Borel set $F \subseteq\left(\mathbf{R}^{d}\right)^{N}$. Then $\left(\otimes_{j=1}^{N} X_{j}\right)\left(\mathbf{R}_{+}^{N}\right) \oplus F$ has positive Lebesgue measure with positive probability if and only if there exists a compact-support Borel probability measure $\mu$ on $F$ such that

$$
\int_{\left(\mathbf{R}^{d}\right)^{N}}\left|\hat{\mu}\left(\xi^{1}, \ldots, \xi^{N}\right)\right|^{2} \prod_{j=1}^{N} \operatorname{Re}\left(\frac{1}{1+\Psi_{j}\left(\xi^{j}\right)}\right) d \xi<\infty .
$$

Next let us suppose that $X_{1}, \ldots, X_{N}$ have one-potential densities $u_{1}, \ldots, u_{N}$, respectively. According to Definition 6.1,

$$
\mathrm{E}\left[\int_{0}^{\infty} f\left(X_{j}(s)\right) e^{-s} d s\right]=\int_{\mathbf{R}^{d}} f(a) u_{j}(a) d a,
$$

for all $j=1, \ldots, N$, and all Borel measurable functions $f: \mathbf{R}^{d} \rightarrow \mathbf{R}_{+}$. [This agrees with the usual nomenclature of probabilistic potential theory.]

Lemma 7.2. If $X_{1}, \ldots, X_{N}$ have respective one-potential densities $u_{1}, \ldots, u_{N}$, then $\otimes_{j=1}^{N} X_{j}$ has the one-potential density $u\left(x^{1}, \ldots, x^{N}\right):=\prod_{j=1}^{N} u_{j}\left(x^{j}\right)$ for all $x^{1}, \ldots, x^{N} \in \mathbf{R}^{d}$. Also, 
$\widetilde{\otimes_{j=1}^{N} X_{j}}$ has the one-potential density

$$
v\left(x^{1}, \ldots, x^{N}\right):=\prod_{j=1}^{N}\left(\frac{u_{j}\left(x^{j}\right)+u_{j}\left(-x^{j}\right)}{2}\right) \quad \text { for all } x^{1}, \ldots, x^{N} \in \mathbf{R}^{d} .
$$

Finally, if $u_{j}(0)>0$ for all $j$, then $u$ and $v$ are strictly positive everywhere.

Proof. Let us prove the first assertion about the one-potential density of $\otimes_{j=1}^{N} X_{j}$ only. The second assertion is proved very similarly.

We seek to establish that for all Borel measureable functions $f:\left(\mathbf{R}^{d}\right)^{N} \rightarrow \mathbf{R}_{+}$,

$$
\mathrm{E}\left[\int_{\mathbf{R}_{+}^{N}} f\left(\left(\otimes_{j=1}^{N} X_{j}\right)(\boldsymbol{t})\right) e^{-[\boldsymbol{t}]} d \boldsymbol{t}\right]=\int_{\left(\mathbf{R}^{d}\right)^{N}} f\left(x^{1}, \ldots, x^{N}\right) \prod_{j=1}^{N} u_{j}\left(x^{j}\right) d x .
$$

A density argument reduces the problem to the case that $f\left(x^{1}, \ldots, x^{N}\right)$ has the special form $\prod_{j=1}^{N} f_{j}\left(x^{j}\right)$, where $f_{j}: \mathbf{R}^{d} \rightarrow \mathbf{R}_{+}$is Borel measurable. But in this case, the claim follows immediately from (17.7) and the independence of $X_{1}, \ldots, X_{N}$.

In order to complete the proof, consider the case that $u_{j}(0)>0$. Lemma 3.2 of Evans (1987b) asserts that $u_{j}(z)>0$ for all $z \in \mathbf{R}^{d}$, whence follows the lemma.

The preceding lemma and Proposition 6.6 together imply, without any further effort, the following two theorems.

Corollary 7.3. Let $X_{1}, \ldots, X_{N}$ be independent Lévy processes on $\mathbf{R}^{d}$, and assume that each $X_{j}$ has a one-potential density $u_{j}$ such that $u_{j}(0)>0$. Then, for all Borel sets $F \subseteq\left(\mathbf{R}^{d}\right)^{N}$,

$$
\mathrm{P}\left\{\left(\otimes_{j=1}^{N} X_{j}\right)\left((0, \infty)^{N}\right) \cap F \neq \varnothing\right\}>0
$$

if and only if there exists a compact-support Borel probability measure $\mu$ on $F$ such that

$$
\int_{\left(\mathbf{R}^{d}\right)^{N}}\left|\hat{\mu}\left(\xi^{1}, \ldots, \xi^{N}\right)\right|^{2} \prod_{j=1}^{N} \operatorname{Re}\left(\frac{1}{1+\Psi_{j}\left(\xi^{j}\right)}\right) d \xi<\infty .
$$

Corollary 7.4 (Fitzsimmons and Salisbury, 1989). Suppose, in addition to the hypotheses of Corollory 7.3, that each $u_{j}: \mathbf{R}^{d} \rightarrow \overline{\mathbf{R}}_{+}$is continuous on $\mathbf{R}^{d}$, and finite on $\mathbf{R}^{d} \backslash\{0\}$. Then, for all Borel sets $F \subseteq\left(\mathbf{R}^{d}\right)^{N}$, (7.10) holds if and only if there exists a compact-support Borel probability measure $\mu$ on $F$ such that

$$
\iint \prod_{j=1}^{N}\left(\frac{u_{j}\left(x^{j}-y^{j}\right)+u_{j}\left(y^{j}-x^{j}\right)}{2}\right) \mu\left(d x^{1} \cdots d x^{N}\right) \mu\left(d y^{1} \cdots d y^{N}\right)<\infty .
$$

We can now prove Theorem 1.3 of the Introduction.

Proof of Theorem 1.3. We observe that: 
(i) $\cap_{j=1}^{N} X_{j}((0, \infty))$ can intersect a set $F \subseteq \mathbf{R}^{d}$ if and only if $\left(\otimes_{j=1}^{N} X_{j}\right)\left((0, \infty)^{N}\right)$ can intersect $G:=\{x \otimes \cdots \otimes x: x \in F\} \subseteq\left(\mathbf{R}^{d}\right)^{N} ;$ and

(ii) any compact-support Borel probability measure $\sigma$ on $G$ manifestly has the form $\sigma\left(d x^{1} \cdots d x^{N}\right)=\mu\left(d x^{1}\right) \prod_{j=2}^{N} \delta_{x^{1}}\left(d x^{j}\right)$, where $\mu$ is a compact-support Borel probability measure on $F$, and hence $\hat{\sigma}\left(\xi^{1}, \ldots, \xi^{N}\right)$ is equal to $\hat{\mu}\left(\xi^{1}+\cdots+\xi^{N}\right)$ for all $\xi^{1}, \ldots, \xi^{N} \in \mathbf{R}^{d}$.

Therefore, Theorem 1.3 is a ready consequence of Corollaries 7.3 and 7.4 .

\section{Proof of Theorem 1.4}

First, we elaborate on the connection between Theorem 1.4 and Bertoin's conjecture (Bertoin, 1999a, p. 49) that was mentioned briefly in the Introduction. Recall that a realvalued Lévy process is a subordinator if its sample functions are monotone a.s. Bertoin, 1996; , 1999a; Fristedt, 1974; Sato, 1999).

Remark 8.1. We consider the special case that $S_{1}$ and $S_{2}$ are two (increasing) subordinators on $\mathbf{R}_{+}$and $F:=\{0\}$, and define two independent Lévy processes by $X_{1}:=S_{1}$ and $X_{2}:=-S_{2}$. Evidently, $X_{1}\left(t_{1}\right)+X_{2}\left(t_{2}\right)=0$ for some $t_{1}, t_{2}>0$ if and only if $S_{1}\left(t_{1}\right)=S_{2}\left(t_{2}\right)$ for some $t_{1}, t_{2}>0$.

Let $\Sigma_{j}$ denote the one-potential measure of $S_{j}$, and suppose $\Sigma_{1}(d x)=u_{1}(x) d x$, where $u_{1}$ is continuous on $\mathbf{R}$ and strictly positive on $(0, \infty)$. Let $U_{j}$ denote the one-potential measure

of $X_{j}$. Then, $U_{1}=\Sigma_{1}$ and $U_{2}=\breve{\Sigma}_{2}$, which we recall is the same as $\Sigma_{2}(-\bullet)$. It follows then that $U_{1}(d x)=u_{1}(x) d x$, and $\left(u_{1} * U_{2}\right)(x)=\int_{0}^{\infty} u_{1}(x+y) \Sigma_{2}(d y)$ is strictly positive a.e. Therefore, we may apply Theorem 1.4 with $F:=\{0\}$ to deduce that

$$
\mathrm{P}\left\{S_{1}\left(t_{1}\right)=S_{2}\left(t_{2}\right) \quad \text { for some } t_{1}, t_{2}>0\right\}>0 \quad \Leftrightarrow \quad Q(0)<\infty .
$$

Because $U_{2}$ does not charge $(-\infty, 0)$ in the present setting,

$$
Q(0):=\int_{0}^{\infty}\left[\frac{u_{1}(y)+u_{1}(-y)}{2}\right] U_{2}(d y)=\frac{1}{2} \int_{0}^{\infty} u_{1}(y) U_{2}(d y),
$$

since $u_{1}(y)=0$ for all $y<0$. [In fact, Lemma 3.2 of Evans (1987b) tells us that $u_{1}(y)=0$ for all $y \leq 0$.] Therefore, we conclude from (8.1) that

$$
\mathrm{P}\left\{S_{1}\left(t_{1}\right)=S_{2}\left(t_{2}\right) \quad \text { for some } t_{1}, t_{2}>0\right\}=0 \quad \Leftrightarrow \quad \int_{0}^{\infty} u_{1}(y) U_{2}(d y)=\infty
$$

Define the zero-potential measures $U_{j}^{0}$ as

$$
U_{j}^{0}(A):=\mathrm{E}\left[\int_{0}^{\infty} \mathbf{1}_{A}\left(S_{j}(t)\right) d t\right],
$$


for $j=1,2$ and Borel sets $A \subseteq \mathbf{R}_{+}$. Suppose $U_{1}^{0}(d x)=u^{0}(x) d x$, where $u^{0}$ is positive and continuous on $(0, \infty)$. Then, it is possible to adapt our methods, without any difficulties, to deduce also that

$$
\mathrm{P}\left\{S_{1}\left(t_{1}\right)=S_{2}\left(t_{2}\right) \quad \text { for some } t_{1}, t_{2}>0\right\}=0 \quad \Leftrightarrow \quad \int_{0}^{\infty} u_{1}^{0}(y) U_{2}^{0}(d y)=\infty .
$$

The end of the proof of Theorem 1.5 contains a discussion which describes how similar changes can be made to adapt the proofs from statements about one-potentials $U_{j}$ to those about zero-potentials $U_{j}^{0}$. We omit the details, as they are not enlightening.

We mention the adaptations to zero-potentials of (8.5) for historical interest: (8.5) was conjectured by Bertoin, under precisely the stated conditions of this remark. Bertoin's conjecture was motivated in part by the fact (Bertoin, 1999b) that, under the very same conditions as above,

$$
\mathrm{P}\left\{S_{1}\left(t_{1}\right)=S_{2}\left(t_{2}\right) \quad \text { for some } t_{1}, t_{2}>0\right\}=0 \quad \Leftrightarrow \quad \sup _{z \in \mathbf{R}} \int_{0}^{\infty} u_{1}^{0}(y+z) U_{2}^{0}(d y)=\infty .
$$

It is possible to deduce (8.6), and the same statement without the zero superscripts, from the present harmonic-analytic methods as well; see Lemma 5.4. The said extension goes well beyond the theory of subordinators, and is a sort of "low intensity maximum principle" (Salisbury, 1992). But we will not describe the details further, since we find the forms of (8.3) and (8.5) simpler to use, as well as easier to conceptualize.

Next we prove Theorem 1.4 without further ado.

Proof of Theorem 1.4. A direct computation reveals that the one-potential density of the two-parameter additive Lévy process $\mathfrak{X}:=X_{1} \oplus X_{2}$ is $u_{1} * U_{2}$. Therefore, the equivalence of (1.14) and (1.15) follows from Part (c) in Proposition 6.6. Next we note that the onepotential density of $\widetilde{X}_{1}$ is described by

$$
v_{1}(x):=\frac{u_{1}(x)+u_{1}(-x)}{2} \quad \text { for all } x \in \mathbf{R}^{d} .
$$

This function is positive a.e. and continuous away from the origin. We can use (6.15) and verify directly that $\widetilde{\mathfrak{X}}$ has a one-potential density $Q$ given by (1.17). Hence the last statement follows from Part (e) of Proposition 6.6.

\section{Intersections of Lévy processes}

The goal of this section is to prove Theorem 1.5. We first return briefly to Theorem 1.3 and discuss how it implies a necessary and sufficient condition for the existence of pathintersections for $N$ independent Lévy processes. After proving Theorem 1.5, we conclude this section by presenting a nontrivial, though simple, example. 
9.1. Existence of intersections. First we develop some general results on equilibrium measure that we believe might be of independent interest.

Choose and fix a compact set $F \subseteq \mathbf{R}^{d}$, and recall that $\operatorname{cap}_{\Psi}(F)$ is the reciprocal of $\inf I_{\Psi}(\mu)$, where the infimum is taken over all Borel probability measures $\mu$ on $F$. It is not hard to see that when $\operatorname{cap}_{\boldsymbol{\Psi}}(F)>0$, this infimum is in fact achieved. Indeed, for all $\epsilon>0$ we can find a Borel probability measure $\mu_{\epsilon}$ on $F$ such that $I_{\Psi}\left(\mu_{\epsilon}\right) \leq(1+\epsilon) / \operatorname{cap}_{\Psi}(F)$. We can extract any subsequential weak limit $\mu$ of $\mu_{\epsilon}$ 's. Evidently, $\mu$ is a Borel probability measure on $F$, and $I_{\Psi}(\mu) \leq 1 / \operatorname{cap}_{\Psi}(F)$ by Fatou's lemma. Because of the defining property of capacity, $I_{\Psi}(\mu) \geq 1 / \operatorname{cap}_{\Psi}(F)$ also. Therefore, $\operatorname{cap}_{\boldsymbol{\Psi}}(F)$ is in fact the reciprocal of the energy of $\mu$.

Any Borel probability measure $\mu$ on $F$ that has the preceding property is called an equilibrium measure on $F$. We now prove that there is only one equilibrium measure on a compact $F$.

Proposition 9.1. If $F \subseteq \mathbf{R}^{d}$ is compact and has positive capacity $\operatorname{cap}_{\mathbf{\Psi}}(F)$, then there exists a unique Borel probability measure $e_{F}$ on $F$ such that $\operatorname{cap}_{\boldsymbol{\Psi}}(F)=1 / I_{\boldsymbol{\Psi}}\left(e_{F}\right)$.

Proof. For all finite signed probability measures $\mu$ and $\nu$ on $\mathbf{R}^{d}$ define

$$
I_{\Psi}(\mu, \nu):=\frac{1}{(2 \pi)^{d}} \int_{\mathbf{R}^{d}}\left(\frac{\overline{\hat{\mu}(\xi)} \hat{\nu}(\xi)+\overline{\hat{\nu}(\xi)} \hat{\mu}(\xi)}{2}\right) K_{\Psi}(\xi) d \xi .
$$

This is well-defined, for example, if $I_{\Psi}(|\mu|)+I_{\Psi}(|\nu|)<\infty$, where $|\mu|$ is the total variation of $\mu$. Indeed, we have the following Cauchy-Schwarz inequality: $\left|I_{\Psi}(\mu, \nu)\right|^{2} \leq I_{\Psi}(|\mu|) \cdot I_{\Psi}(|\nu|)$. If $\sigma$ is a finite [nonnegative] Borel measure on $\mathbf{R}^{d}$, then $I_{\Psi}(\sigma, \sigma)$ agrees with $I_{\Psi}(\sigma)$, and this is positive as long as $\sigma$ is not the zero measure. However, we may note that slightly more general fact that if $\sigma$ is a non-zero finite signed measure, then we still have

$$
I_{\Psi}(\sigma, \sigma)>0
$$

This follows from the fact that $K_{\Psi}(\xi)>0$ for all $\xi \in \mathbf{R}^{d}$.

Let $c:=1 / \operatorname{cap}_{\Psi}(F)$, and suppose $\mu$ and $\nu$ were two distinct equilibrium measures on $F$. That is, $\mu \neq \nu$ but $I_{\Psi}(\mu)=I_{\Psi}(\nu)=1 / c$. In accord with (9.2),

$$
0<I_{\Psi}\left(\frac{\mu-\nu}{2}, \frac{\mu-\nu}{2}\right)=\frac{c-I_{\Psi}(\mu, \nu)}{2} .
$$


Consequently, $I_{\Psi}(\mu, \nu)<c$, and hence

$$
\begin{aligned}
I_{\Psi}\left(\frac{\mu+\nu}{2}\right) & =\frac{1}{4} I_{\Psi}(\mu)+\frac{1}{4} I_{\Psi}(\nu)+\frac{1}{2} I_{\Psi}(\mu, \nu) \\
& =\frac{c+I_{\Psi}(\mu, \nu)}{2} \\
& <c .
\end{aligned}
$$

Because $\frac{1}{2}(\mu+\nu)$ is a Borel probability measure on $F$, this is contradicts the fact that $c$ is the smallest possible energy on $F$.

Next we note with the following computation of the equilibrium measure in a specific class of examples. The following result is related quite closely to the celebrated local ergodic theorem of Csiszár (1965). [We hope to elaborate on this connection elsewhere.] See also Proposition A3 of Khoshnevisan, Xiao, and Zhong (2003) for a related result.

Proposition 9.2. Suppose $F$ is a fixed compact subset of $\mathbf{R}^{d}$ with a nonvoid interior. If $\operatorname{cap}_{\Psi}(F)>0$, then $e_{F}$ is the normalized Lebesgue measure on $F$.

Proof. Our strategy is to prove that $e_{F}$ is translation invariant.

Let $\mathscr{R}(r)$ denote the collection of all closed "upright" cubes of the form

$$
I:=\left[s_{1}, s_{1}+r\right] \times \cdots \times\left[s_{d}, s_{d}+r\right] \subseteq F
$$

such that all of the $s_{i}$ 's are rational numbers and $r>0$. Each $\mathscr{R}(r)$ is a countable collection, and hence we can (and will) enumerate its elements as $I_{1}(r), I_{2}(r), \ldots$.

For all $i, j \geq 1$ and $r>0$ we choose and fix a one-to-one onto piecewise-linear map $\theta_{i, j, r}: F \rightarrow F$ that has the following properties:

- If $a \notin I_{i}(r) \cup J_{j}(r)$, then $\theta_{i, j, r}(a)=a$;

- $\theta_{i, j, r}$ maps $I_{i}(r)$ onto $J_{i}(r)$ bijectively; and

- $\theta_{i, j, r}$ maps $J_{i}(r)$ onto $I_{i}(r)$ bijectively.

To be concrete, let us write $I_{i}(r)=I_{j}(r)+b$, where $b \in \mathbf{R}^{d}$. Then we define

$$
\theta_{i, j, r}(a)= \begin{cases}a-b & \text { if } a \in I_{i}(r), \\ a+b & \text { if } a \in I_{j}(r), \\ a & \text { if } a \notin I_{i}(r) \cup J_{i}(r) .\end{cases}
$$

It can be verified that $\theta_{i, j, r} \circ \theta_{i, j, r}=i d$, the identity map.

For all integers $n \geq 1$ and $r>0$ consider

$$
\rho_{n, r}:=\frac{1}{n^{2}} \sum_{1 \leq i, j \leq n} \sum_{F}\left(e_{F} \circ \theta_{i, j, r}^{-1}\right) .
$$


Obviously, each $\rho_{n, r}$ is a probability measure on $F$, and

$$
\rho_{n, r}\left(I_{i}(r)\right)=\rho_{n, r}\left(I_{j}(r)\right) \quad \text { for all } 1 \leq i, j \leq n .
$$

Furthermore, a direct computation reveals that for all $i, j \geq 1$ and $r>0$,

$$
\left|\widehat{e_{F} \circ \theta_{i, j, r}^{-1}}\right|=\left|\widehat{e_{F}}\right| \quad \text { pointwise. }
$$

Therefore, we can apply Minkowski's inequality, to the norm $\mu \mapsto \sqrt{I_{\Psi}(\mu)}$, to find that

$$
\begin{aligned}
\sqrt{I_{\Psi}\left(\rho_{n, r}\right)} & \leq \frac{1}{n^{2}} \sum_{1 \leq i, j \leq n} \sqrt{I_{\Psi}\left(e_{F} \circ \theta_{i, j, r}^{-1}\right)} \\
& =\sqrt{I_{\Psi}\left(e_{F}\right)} .
\end{aligned}
$$

By the uniqueness of equilibrium measure (Proposition 9.1), $e_{F}=\rho_{n, r}$ for all $n \geq 1$ and rationals $r>0$. This and (9.8) together prove that $e_{F}(I)=e_{F}(J)$ for all $r>0$ and all $I, J \in \mathscr{R}(r)$. A monotone-class argument reveals that for all Borel sets $I \subset F$ and $b \in \mathbf{R}^{d}$, $e_{F}(I)=e_{F}(b+I)$, provided that $I, b+I \subseteq F$. Because the Lebesgue measure is characterized by its translation invariance, this implies the proposition.

Lemma 5.6 and Proposition [9.2, and Theorem 1.3 together imply the following variant of a theorem of Fitzsimmons and Salisbury (1989).

Corollary 9.3. Let $X_{1}, \ldots, X_{N}$ be independent Lévy processes on $\mathbf{R}^{d}$, and assume that each $X_{j}$ has a one-potential density $u_{j}$ that is continuous on $\mathbf{R}^{d}$, positive at zero, and finite on $\mathbf{R}^{d} \backslash\{0\}$. Then,

$$
\mathrm{P}\left\{X_{1}\left(t_{1}\right)=\cdots=X_{N}\left(t_{N}\right) \text { for some } t_{1}, \ldots, t_{N}>0\right\}>0
$$

if and only if

$$
\prod_{j=1}^{N}\left(\frac{u_{j}(\bullet)+u_{j}(-\bullet)}{2}\right) \in L_{l o c}^{1}\left(\mathbf{R}^{d}\right)
$$

Proof. Clearly, (9.11) holds if and only if there exists $n>0$ such that

$$
\mathrm{P}\left\{X_{1}\left(t_{1}\right)=\cdots=X_{N}\left(t_{N}\right) \in[-n, n]^{d} \text { for some } t_{1}, \ldots, t_{N}>0\right\}>0 .
$$

Theorem 1.3 implies that (9.13) holds if and only if $\operatorname{cap}_{\boldsymbol{\Psi}}\left([-n, n]^{d}\right)>0$. Lemma [5.6] and Proposition 9.2 together prove the result.

9.2. Proof of Theorem 1.5. We begin by proving the first part; thus, we assume only that the $u_{j}$ 's exist and are a.e.-positive. 
Let $\mathfrak{S}$ denote an independent $M$-parameter additive stable process of index $\alpha \in(0,2)$; see (10.9). Next, we consider the $(N+M)$-parameter process $\mathfrak{Y}:=\otimes_{j=1}^{N}\left(X_{j}-\mathfrak{S}\right)$; i.e.,

$$
\mathfrak{Y}(\boldsymbol{s} \otimes \boldsymbol{t}):=\left(\begin{array}{c}
X_{1}\left(s_{1}\right)-\mathfrak{S}(\boldsymbol{t}) \\
\vdots \\
X_{N}\left(s_{N}\right)-\mathfrak{S}(\boldsymbol{t})
\end{array}\right) \quad \text { for all } \quad \boldsymbol{s} \in \mathbf{R}_{+}^{N}, \boldsymbol{t} \in \mathbf{R}_{+}^{M} .
$$

It is not hard to adapt the discussion of the first few paragraphs in $\$ 7$ to the present situation and deduce that $\mathfrak{Y}$ is an $(N+M)$-parameter additive Lévy process, with values in $\left(\mathbf{R}^{d}\right)^{N}$, and that for all $s \in \mathbf{R}_{+}^{N}, \boldsymbol{t} \in \mathbf{R}_{+}^{M}$, and $\xi:=\xi^{1} \otimes \cdots \otimes \xi^{N} \in\left(\mathbf{R}^{d}\right)^{N}$,

$$
\operatorname{Eexp}(i \xi \cdot \mathfrak{Y}(\boldsymbol{s} \otimes \boldsymbol{t}))=\exp \left(-\sum_{k=1}^{N} s_{k} \Psi_{k}\left(\xi^{k}\right)-\sum_{l=1}^{M} t_{l}\left\|\xi^{1}+\cdots+\xi^{N}\right\|^{\alpha}\right) .
$$

We can conclude readily from this that the characteristic exponent of $\mathfrak{Y}$ is defined by

$$
\boldsymbol{\Theta}(\xi):=(\Psi_{1}\left(\xi^{1}\right), \ldots, \Psi_{N}\left(\xi^{N}\right), \underbrace{\left\|\sum_{j=1}^{N} \xi^{j}\right\|^{\alpha}, \cdots,\left\|\sum_{j=1}^{N} \xi^{j}\right\|^{\alpha}}_{M \text { times }}),
$$

for all $\xi:=\xi^{1} \otimes \cdots \otimes \xi^{N} \in\left(\mathbf{R}^{d}\right)^{N}$.

It follows readily from this and Lemma 7.2 that $\mathfrak{Y}$ and $\widetilde{\mathfrak{Y}}$ both have positive one-potential densities. Moreover, a direct computation involving the inversion formula reveals that the potential density of $\widetilde{\mathfrak{Y}}$ is defined by

$$
v\left(x^{1} \otimes \cdots \otimes x^{N}\right)=\int_{\mathbf{R}^{d}} \prod_{j=1}^{N}\left(\frac{u_{j}\left(x^{j}-y\right)+u_{j}\left(y-x^{j}\right)}{2}\right) w(y) d y,
$$

for all $x:=\left(x^{1} \otimes \cdots \otimes x^{N}\right) \in\left(\mathbf{R}^{d}\right)^{N}$. Here, $w$ denotes the one-potential density of $\mathfrak{S}$. That is,

$$
w(y):=\int_{\mathbf{R}_{+}^{M}} p_{\boldsymbol{t}}(y) e^{-[t]} d \boldsymbol{t}
$$

where $p_{\boldsymbol{t}}$ denotes the density of $\mathfrak{S}(\boldsymbol{t})$. That is, $p_{\boldsymbol{t}}(y):=(2 \pi)^{-d} \int_{\mathbf{R}^{d}} \exp \left(-i y \cdot z-[\boldsymbol{t}]\|z\|^{\alpha}\right) d z$ for all $\boldsymbol{t} \in \mathbf{R}_{+}^{M}$ and $y \in \mathbf{R}^{d}$.

Since the $u_{j}$ 's are everywhere positive (Lemma 7.2), Proposition 6.6 tell us that $0 \in$ $\mathfrak{Y}\left(\mathbf{R}_{+}^{N} \times \mathbf{R}_{+}^{M}\right)$ with positive probability if and only if $\operatorname{cap}_{\boldsymbol{\Theta}}(\{0\})>0$. Thus, the preceding 
positive capacity condition is equivalent to the integrability of the function $K_{\boldsymbol{\Theta}}$. That is,

$$
\begin{aligned}
0 \in \mathfrak{Y} & \left(\mathbf{R}_{+}^{N} \times \mathbf{R}_{+}^{M}\right) \quad \text { with positive probability } \\
& \Leftrightarrow \quad \int_{\left(\mathbf{R}^{d}\right)^{N}} \prod_{j=1}^{N} \operatorname{Re}\left(\frac{1}{1+\Psi_{j}\left(\xi^{j}\right)}\right) \frac{d \xi}{1+\left\|\xi^{1}+\cdots+\xi^{N}\right\|^{\alpha M}}<\infty .
\end{aligned}
$$

On the other hand, it is manifestly the case that $\mathfrak{Y}\left(\mathbf{R}_{+}^{N} \times \mathbf{R}_{+}^{M}\right)$ contains the origin if and only if the intersection of $\cap_{j=1}^{N} X_{j}\left(\mathbf{R}_{+}\right)$and $\mathfrak{S}\left(\mathbf{R}_{+}^{M}\right)$ is nonempty. Thanks to Theorem 4.4 .1 of Khoshnevisan (2002, p. 428), for all Borel sets $F \subseteq \mathbf{R}^{d}, \mathrm{P}\left\{\mathfrak{S}\left(\mathbf{R}_{+}^{M}\right) \cap F \neq \varnothing\right\}>0$ if and only $\mathscr{C}_{d-M \alpha}(F)>0$, provided that we also assume that $d>M \alpha$. Suppose, then, that $d>M \alpha$. We can apply the preceding, conditionally on $X_{1}, \ldots, X_{N}$, and deduce that

$$
\begin{aligned}
0 \in \mathfrak{Y} & \left(\mathbf{R}_{+}^{N} \times \mathbf{R}_{+}^{M}\right) \quad \text { with positive probability } \\
& \Leftrightarrow \quad \mathscr{C}_{d-M \alpha}\left(\bigcap_{j=1}^{N} X_{j}\left(\mathbf{R}_{+}\right)\right)>0 \quad \text { with positive probability. }
\end{aligned}
$$

We compare the preceding display to (9.19), and choose $M$ and $\alpha \in(0,(d / M) \wedge 2)$, such that $d-M \alpha$ is any predescribed number $s \in(0, d)$. This yields the following: For all $s \in(0, d)$,

$$
\begin{aligned}
& \mathscr{C}_{s}\left(\bigcap_{j=1}^{N} X_{j}\left(\mathbf{R}_{+}\right)\right)>0 \quad \text { with positive probability } \\
& \quad \Leftrightarrow \quad \int_{\left(\mathbf{R}^{d}\right)^{N}} \prod_{j=1}^{N} \operatorname{Re}\left(\frac{1}{1+\Psi_{j}\left(\xi^{j}\right)}\right) \frac{d \xi}{1+\left\|\xi^{1}+\cdots+\xi^{N}\right\|^{d-s}}<\infty .
\end{aligned}
$$

It follows from (9.21), Frostman's theorem (Khoshnevisan, 2002, p. 521) and an argument similar to the proof of Theorem 3.2 in Khoshnevisan, Shieh and Xiao (2007) that the first identity of (1.18) holds almost surely on $\left\{\cap_{j=1}^{N} X_{j}\left(\mathbf{R}_{+}\right) \neq \varnothing\right\}$.

In order to obtain (1.19), we assume also that the $u_{j}$ 's are continuous on $\mathbf{R}^{d}$ and finite on $\mathbf{R}^{d} \backslash\{0\}$. Thanks to Proposition 6.6, $\mathfrak{Y}\left(\mathbf{R}_{+}^{N} \times \mathbf{R}_{+}^{M}\right)$ contains zero with positive probability if and only if $v(0)<\infty$. Thus, (9.17) and (9.20) imply that

$$
\begin{gathered}
\mathscr{C}_{d-M \alpha}\left(\bigcap_{j=1}^{N} X_{j}\left(\mathbf{R}_{+}\right)\right)>0 \text { with positive probability } \\
\Leftrightarrow \int_{\mathbf{R}^{d}} \prod_{j=1}^{N}\left(\frac{u_{j}(y)+u_{j}(-y)}{2}\right) w(y) d y<\infty
\end{gathered}
$$

If we could replace $w(y)$ by $\|y\|^{-d+\alpha M}$, then we could finish the proof by choosing $M$ and $\alpha$ suitably, and then appealing to the Frostman theorem and the argument in Khoshnevisan, 
Shieh and Xiao (2007). [This is how we completed the proof of the first part of the proof as well.] Thus, our goal is to derive (9.22).

Unfortunately, it is not possible to simply replace $w(y)$ by $\|y\|^{-d+\alpha M}$ by simple real-variable arguments. Nonetheless, we recall the following fact from Khoshnevisan (2002, Exercise 4.1.4, p. 423): There exist $c, C \in(0, \infty)$ such that $c\|y\|^{-d+M \alpha} \leq w(y) \leq C\|y\|^{-d+M \alpha}$ for all $y \in(-1,1)^{d}$. Moreover, the upper bound holds for all $y \in \mathbf{R}^{d}$ (loc. cit., Eq. (2), p. 423); the lower bound [provably] does not. It follows then that

$$
\begin{aligned}
\int_{\mathbf{R}^{d}} & \prod_{j=1}^{N}\left(\frac{u_{j}(y)+u_{j}(-y)}{2}\right) \frac{d y}{\|y\|^{d-M \alpha}}<\infty \\
& \Rightarrow \mathscr{C}_{d-M \alpha}\left(\bigcap_{j=1}^{N} X_{j}\left(\mathbf{R}_{+}\right)\right)>0 \quad \text { with positive probability } \\
& \Rightarrow \quad \int_{(-1,1)^{d}} \prod_{j=1}^{N}\left(\frac{u_{j}(y)+u_{j}(-y)}{2}\right) \frac{d y}{\|y\|^{d-M \alpha}}<\infty
\end{aligned}
$$

If we could replace $(-1,1)^{d}$ by $\mathbf{R}^{d}$ in the last display, then our proof follows the outline mentioned earlier. Thus, we merely point out how to derive (9.22), with $w(y)$ replaced by $\|y\|^{-d+M \alpha}$, and omit the remainder of the argument.

The proof hinges on a few modifications to the entire theory outlined here. We describe them [very] briefly, since it is easy-though tedious - to check that the present changes go through unhindered.

Define the operator $R^{1 \otimes 0}$ via

$$
\left(R^{1 \otimes 0} f\right)(x):=\frac{1}{2^{N}} \mathrm{E}\left[\int_{\mathbf{R}^{N} \times \mathbf{R}^{M}} f(x+\widetilde{\mathfrak{Y}}(\boldsymbol{s} \otimes \boldsymbol{t})) e^{-[\boldsymbol{s}]} d \boldsymbol{s} d \boldsymbol{t}\right] \quad \text { for all } x \in\left(\mathbf{R}^{d}\right)^{N} .
$$

If, we replace $\exp (-[\boldsymbol{s}])$ by $\exp (-[\boldsymbol{s}]-[\boldsymbol{t}])$, then $R^{1 \otimes 0} f$ turns into $R f$.

As is, the operator $R^{1 \otimes 0} f$ fails to map $L^{p}\left(\left(\mathbf{R}^{d}\right)^{N}\right)$ into $L^{p}\left(\left(\mathbf{R}^{d}\right)^{N}\right)$. But this is a minor technical nuisance, since one can check directly that

$$
\left(R^{1 \otimes 0} f\right)(x)=\int_{\mathbf{R}^{d}} f(x+y) v^{1 \otimes 0}(y) d y,
$$

where $v^{1 \otimes 0}$ is defined exactly as $v$ was, but with $w(y)$ replaced by a certain constant times $\|y\|^{-d+M \alpha}$. And this shows fairly readily that if $f$ is a compactly-supported function in $L^{1}\left(\left(\mathbf{R}^{d}\right)^{N}\right)$, then $R^{1 \otimes 0} f \in L_{l o c}^{1}\left(\left(\mathbf{R}^{d}\right)^{N}\right)$. To complete our proof, we need to redevelop the potential theory of the additive Lévy process $\mathfrak{Y}$, but this time in terms of $R^{1 \otimes 0} f$ and $v^{1 \otimes 0}$.

The fact that $R^{1 \otimes 0}: L_{c}^{1}\left(\left(\mathbf{R}^{d}\right)^{N}\right) \rightarrow L_{l o c}^{1}\left(\left(\mathbf{R}^{d}\right)^{N}\right)$ provides sufficient regularity to allow us to push the Fourier analysis of the present paper through without change. And the end result 
is that $\mathfrak{Y}\left(\mathbf{R}_{+}^{N} \times \mathbf{R}_{+}^{M}\right)$ contains zero if and only if $v^{1 \otimes 0}(0)$ is finite. Now we can complete the proof, but with $v^{1 \otimes 0}$ in place of $v$ everywhere.

9.3. An example. Suppose $X_{1}, \ldots, X_{N}$ are independent isotropic stable processes in $\mathbf{R}^{d}$ with respective Fourier transforms $\operatorname{E} \exp \left(i \xi \cdot X_{j}(t)\right)=\exp \left(-c_{j} t\|\xi\|^{\alpha_{j}}\right)$ for all $t \geq 0, \xi \in \mathbf{R}^{d}$, and $1 \leq j \leq N$. Here, $c_{1}, \ldots, c_{N}$ are constants, and $0<\alpha_{1}, \ldots, \alpha_{N}<2 \wedge d$ are the indices of stability. We are primarily interested in the case that $N \geq 2$, but the following remarks apply to the case $N=1$ equally well.

In this section we work out some of intersection properties of $X_{1}, \ldots, X_{N}$. It is possible to construct much more sophisticated examples. We study the present setting because it provides us with the simplest nontrivial example of its type.

It is known that each $X_{j}$ has a continuous positive one-potential density $u_{j}$, and there exist $c, C \in(0, \infty)$ such that $c\|x\|^{-d+\alpha_{j}} \leq u_{j}(x) \leq C\|x\|^{-d+\alpha_{j}}$ for all $x \in(-1,1)^{d}$ and $1 \leq j \leq N$. [These assertions follow, for example, from Corollary 3.2.1 on page 379, and Lemma 3.4.1 on page 383 of Khoshnevisan (2002).] Consequently, Theorem 1.3 immediately implies that for all Borel sets $F \subseteq \mathbf{R}^{d}$,

$$
\mathrm{P}\left\{\bigcap_{j=1}^{N} X_{j}\left(\mathbf{R}_{+}\right) \cap F \neq \varnothing\right\}>0 \Leftrightarrow \mathscr{C}_{N d-\sum_{j=1}^{N} \alpha_{j}}(F)>0 .
$$

In the case that $N=2$, a slightly more general form of this was found in Khoshnevisan (2002, Theorem 4.4.1, p. 428) by using other methods. We may apply the preceding with $F=\mathbf{R}^{d}$, and appeal to Taylor's theorem (loc. cit., Corollary 2.3.1, p. 525) to find that

$$
\mathrm{P}\left\{\bigcap_{k=1}^{N} X_{k}\left(\mathbf{R}_{+}\right) \neq \varnothing\right\}>0 \Leftrightarrow(N-1) d<\sum_{j=1}^{N} \alpha_{j} .
$$

Theorem 1.5, and a direct computation in polar coordinates, together show that the slack in the preceding inequality determines the Hausdorff dimension of the set $\cap_{k=1}^{N} X_{k}\left(\mathbf{R}_{+}\right)$of intersection points. That is, almost surely on $\left\{\cap_{k=1}^{N} X_{k}\left(\mathbf{R}_{+}\right) \neq \varnothing\right\}$,

$$
\operatorname{dim}_{\mathrm{H}} \bigcap_{k=1}^{N} X_{k}\left(\mathbf{R}_{+}\right)=\left[\sum_{k=1}^{N} \alpha_{k}-(N-1) d\right]_{+} .
$$

This formula continues to hold in case some, or even all, of the $\alpha_{j}$ 's are equal to or exceed $d$. We omit the details.

9.4. Remarks on multiple points. Next we mention how the preceding fits in together with the well-known conjecture of Hendricks and Taylor (1979) that was solved in Fitzsimmons and Salisbury (1989), and also make a few related remarks. 
Remark 9.4. Recall that a [single] Lévy process $X$ with values in $\mathbf{R}^{d}$ has $N$-multiple points if and only if there exist times $0<t_{1}<\ldots<t_{N}<\infty$ such that $X\left(t_{1}\right)=X\left(t_{2}\right)=\cdots=X\left(t_{N}\right)$. [We are ruling out the possibility that $t_{1}=0$ merely to avoid degeneracies.]

By localization and the Markov property, $X$ has $N$-multiple points almost surely if and only if there are times $t_{1}, \ldots, t_{N} \in(0, \infty)$ such that $X_{1}\left(t_{1}\right)=\cdots=X_{N}\left(t_{N}\right)$ with positive probability, where $X_{1}, \ldots, X_{N}$ are $N$ i.i.d. copies of $X$.

Suppose that $X$ has a a continuous and positive [equivalently, positive-at-zero] onepotential density $u$ that is finite on $\mathbf{R}^{d} \backslash\{0\}$. Then according to Corollary $9.3, X$ has $N$-multiple points if and only if $u(\bullet)+u(-\bullet) \in L_{l o c}^{N}\left(\mathbf{R}^{d}\right)$. An application of Hölder's inequality reveals then that $X$ has $N$-multiple points if and only if $u \in L_{l o c}^{N}\left(\mathbf{R}^{d}\right)$. This is more or less the well-known condition of Hendricks and Taylor (1979).

More generally, the following can be deduced with no extra effort: Under the preceding conditions, given a nonrandom Borel set $F \subseteq \mathbf{R}^{d}$,

$$
\mathrm{P}\left\{\text { there exist } 0<t_{1}<\cdots<t_{N} \text { such that } X\left(t_{1}\right)=\cdots=X\left(t_{N}\right) \in F\right\}>0
$$

if and only if there exists a compact-support Borel probability measure $\mu$ on $F$ such that

$$
\iint[u(x-y)]^{N} \mu(d x) \mu(d y)<\infty .
$$

See, for example, Theorem 5.1 of Fitzsimmons and Salisbury (1989).

Remark 9.5. We mention the following formula for the Hausdorff dimension of the $N$-multiple points of a Lévy process $X$ : Under the conditions stated in Remark 9.4,

$$
\operatorname{dim}_{\mathrm{H}} M_{N}=\sup \left\{s \in(0, d): \int_{\mathbf{R}^{d}} \frac{[u(z)]^{N}}{\|z\|^{s}} d z<\infty\right\} \quad \text { a.s. }
$$

where $M_{N}$ denotes the collection of all $N$-multiple points. That is, $M_{N}$ is the set of all $x \in \mathbf{R}^{d}$ for which the cardinality of $X^{-1}(\{x\})$ is at least $N$. This formula appears to be new. Hawkes (1978b, Theorem 2) contains a similar formula - with $\int_{\mathbf{R}^{d}}$ replaced by $\int_{(-1,1)^{d}}$ which is shown to be valid for all isotropic [spherically symmetric] Lévy processes that have measurable transition densities.

In order to prove (9.31), we appeal to Theorem 1.5 and the Markov property to first demonstrate that the dimension formula in (9.31) is valid almost surely on $\left\{M_{N} \neq \varnothing\right\}$. Then the conclusion follows from the fact that the event $\left\{M_{N} \neq \varnothing\right\}$ satisfies a zero-one law; that zero-one law is itself proved by adapting the argument of Orey (1967, p. 124) or Evans (1987b, pp. 365-366). We omit the details as the method is nowadays considered standard. 
Remark 9.6. This is a natural place to complete a computation that we alluded to earlier in Open Problem 4, Namely, we wish to prove that under the preceding conditions on the Lévy process $X$,

$$
\operatorname{dim}_{\mathrm{H}} M_{2}=\sup \left\{s \in(0, d): \int_{(-1,1)^{d}} \frac{[u(z)]^{2}}{\|z\|^{s}} d z<\infty\right\} \quad \text { a.s. }
$$

By the Markov property, it suffices to derive (9.32) with $M_{2}$ replaced by $X_{1}\left(\mathbf{R}_{+}\right) \cap X_{2}\left(\mathbf{R}_{+}\right)$, where $X_{1}$ and $X_{2}$ are independent copies of $X$.

We have seen already that if $u \notin L_{l o c}^{2}\left(\mathbf{R}^{d}\right)$, then $X_{1}\left(\mathbf{R}_{+}\right) \cap X_{2}\left(\mathbf{R}_{+}\right)=\varnothing$, and there is nothing left to prove. Thus, we may consider only the case that $u \in L_{l o c}^{2}\left(\mathbf{R}^{d}\right)$. In this case, $X_{1}\left(\mathbf{R}_{+}\right) \cap X_{2}\left(\mathbf{R}_{+}\right) \neq \varnothing$, which as we have seen is equivalent to the condition that $\left(X_{1} \ominus X_{2}\right)\left(\mathbf{R}_{+}^{2}\right)$ contains the origin. This and Theorem 1.1 together prove that $K_{\Psi} \in L^{2}\left(\mathbf{R}^{d}\right)$, where $K_{\Psi}(\xi)=\operatorname{Re}(1+\Psi(\xi))^{-1}$ and $\operatorname{E} \exp (i \xi \cdot X(t))=\exp (-t \Psi(\xi))$ in the present case. Now Lemma 6.2 shows that $\hat{v}$-and hence $v$-is square integrable, where $v:=\frac{1}{2}(u(\bullet)+u(-\bullet))$. That is, $u \in L^{2}\left(\mathbf{R}^{d}\right)$. From here, it is a simple matter to check that (9.31) with $N=2$ implies (9.32).

\section{Zero-one laws}

We conclude this paper by deriving two zero-one laws: One for the Lebesgue measure of the range of additive Lévy processes; and another for capacities of the range of additive Lévy processes.

The following was proved first in Khoshnevisan, Xiao, and Zhong (2003) under a mild technical condition. Here we remove the technical condition (1.3) of that paper, and derive this result as an elementary consequence of Theorem 1.1.

Proposition 10.1. Let $\mathfrak{X}$ be a general $N$-parameter additive Lévy process in $\mathbf{R}^{d}$ with exponent $\boldsymbol{\Psi}$. Then,

$$
\mathrm{E}\left[\lambda_{d}\left(\mathfrak{X}\left(\mathbf{R}_{+}^{N}\right)\right)\right]>0 \quad \text { if and only if } \int_{\mathbf{R}^{d}} \prod_{j=1}^{N} \operatorname{Re}\left(\frac{1}{1+\Psi_{j}(\xi)}\right) d \xi<\infty .
$$

Suppose, in addition, that $\widetilde{\mathfrak{X}}$ has a one-potential density that is continuous away from the origin, and $\mathfrak{X}$ has an a.e.-positive one-potential density. Then, with probability one, $\lambda_{d}\left(\mathfrak{X}\left(\mathbf{R}_{+}^{N}\right)\right)$ is zero or infinity.

Proof. According to Theorem 1.1, $\mathrm{E}\left[\lambda_{d}\left(\mathfrak{X}\left(\mathbf{R}_{+}^{N}\right)\right)\right]$ is positive if and only if $\operatorname{cap}_{\boldsymbol{\Psi}}(\{0\})>0$. But the only probability measure on $\{0\}$ is $\delta_{0}$, and hence $\operatorname{cap}_{\Psi}(\{0\})>0$ iff $K_{\Psi} \in L^{1}\left(\mathbf{R}^{d}\right)$. This is the integrability condition of (10.1), and implies the assertion made in (10.1). 
Now the proof of Proposition 6.5 of Khoshnevisan et al. (2003) goes through unhindered to conclude the remainder of the proof. We include it here for the sake of completeness. Throughout the rest of this proof, we assume that $\mathfrak{X}$ has an a.e.-positive one-potential density.

Our task is to prove that if $\mathrm{E}\left[\lambda_{d}\left(\mathfrak{X}\left(\mathbf{R}_{+}^{N}\right)\right)\right]$ is finite, then it is zero. Note that for all $n>0$,

$$
\begin{aligned}
& \mathrm{E}\left[\lambda_{d}\left(\mathfrak{X}\left(\mathbf{R}_{+}^{N}\right)\right)\right] \\
& \geq \mathrm{E}\left[\lambda_{d}\left(\mathfrak{X}\left([0, n]^{N}\right)\right)\right]+\mathrm{E}\left[\lambda_{d}\left(\mathfrak{X}\left([n, \infty)^{N}\right)\right)\right]-\mathrm{E}\left[\lambda_{d}\left(\mathfrak{X}\left([0, n]^{N}\right) \cap \mathfrak{X}^{\prime}\left(\mathbf{R}_{+}^{N}\right)\right)\right],
\end{aligned}
$$

where $\mathfrak{X}^{\prime}$ is an independent copy of $\mathfrak{X}$. Therefore, if $\mathrm{E}\left[\lambda_{d}\left(\mathfrak{X}\left(\mathbf{R}_{+}^{N}\right)\right)\right]$ is finite, then

$$
\mathrm{E}\left[\lambda_{d}\left(\mathfrak{X}\left([0, n]^{N}\right)\right] \leq \mathrm{E}\left[\lambda_{d}\left(\mathfrak{X}\left([0, n]^{N}\right) \cap \mathfrak{X}^{\prime}\left(\mathbf{R}_{+}^{N}\right)\right)\right] .\right.
$$

The inequality is, in fact, an equality. Let $n \uparrow \infty$ to deduce that if $\mathrm{E}\left[\lambda_{d}\left(\mathfrak{X}\left(\mathbf{R}_{+}^{N}\right)\right)\right]$ is finite, then $\mathrm{E}\left[\lambda_{d}\left(\mathfrak{X}\left(\mathbf{R}_{+}^{N}\right)\right)\right]=\mathrm{E}\left[\lambda_{d}\left(\mathfrak{X}\left(\mathbf{R}_{+}^{N}\right) \cap \mathfrak{X}^{\prime}\left(\mathbf{R}_{+}^{N}\right)\right)\right]$. Consider the function

$$
\phi(a):=\mathrm{P}\left\{a \in \mathfrak{X}\left(\mathbf{R}_{+}^{N}\right)\right\} \quad \text { for all } a \in \mathbf{R}^{d} .
$$

Then, we have just proved that $\int_{\mathbf{R}^{d}} \phi(a) d a=\int_{\mathbf{R}^{d}}|\phi(a)|^{2} d a$. Since $0 \leq \phi(a)(1-\phi(a)) \leq 1$ for all $a \in \mathbf{R}^{d}$, it follows that $\phi \in\{0,1\}$ almost everywhere. Consequently, if $\mathrm{E}\left[\lambda_{d}\left(\mathfrak{X}\left(\mathbf{R}_{+}^{N}\right)\right)\right]$ is finite, then

$$
\mathrm{E}\left[\lambda_{d}\left(\mathfrak{X}\left(\mathbf{R}_{+}^{N}\right)\right)\right]=\lambda_{d}\left(\phi^{-1}(\{1\})\right) .
$$

According to Proposition [6.6, either $\phi(a)=0$ for all $a$, or $\phi(a)=1$ for all $a$. Since $\mathrm{E}\left[\lambda_{d}\left(\mathfrak{X}\left(\mathbf{R}_{+}^{N}\right)\right)\right]<\infty$, this and (10.5) together prove that $\phi(a)=0$ for all $a$, and hence $\mathrm{E}\left[\lambda_{d}\left(\mathfrak{X}\left(\mathbf{R}_{+}^{N}\right)\right)\right]=0$.

For all $s>0$ define $\mathscr{C}_{s}(A)$ to be the $s$-dimensional Bessel-Riesz capacity of the Borel set $A \subseteq \mathbf{R}^{d}$. That is,

$$
\mathscr{C}_{s}(A):=\left[\inf _{\mu \in \mathscr{P}_{c}(A)} \iint \frac{\mu(d x) \mu(d y)}{\|x-y\|^{s}}\right]^{-1},
$$

where inf $\varnothing:=\infty, 1 / \infty:=0$, and we recall that $\mathscr{P}_{c}(A)$ denotes the collection of all compactsupport Borel probability measures on $A$. Thus, $\mathscr{C}_{s}=\mathscr{C}_{\kappa_{d-s}}$, where $\kappa_{\alpha}(x):=\|x\|^{-d+\alpha}$ denotes the $(d-\alpha)$-dimensional Riesz kernel. The goal of this subsection is to derive a zero-one law for the capacity of the range of an arbitrary additive Lévy process $\mathfrak{X}$.

Proposition 10.2. If $\mathfrak{X}$ denotes an $N$-parameter additive Lévy process in $\mathbf{R}^{d}$, then for all $\beta \in(0, d)$ fixed, the chances are either zero or one that $\mathscr{C}_{\beta}\left(\mathfrak{X}\left(\mathbf{R}_{+}^{N}\right)\right)$ is strictly positive.

The following formula for the Hausdorff dimension of $\mathfrak{X}\left(\mathbf{R}_{+}^{N}\right)$ is an immediate application of Proposition 10.2 and the methods of Khoshnevisan and Xiao (2004, Theorem 4.1): If $\mathfrak{X}$ is 
an additive Lévy process with values in $\mathbf{R}^{d}$ with characteristic exponent $\left(\Psi_{1}, \ldots, \Psi_{N}\right)$, then almost surely,

$$
\operatorname{dim}_{\mathrm{H}}\left(\mathfrak{X}\left(\mathbf{R}_{+}^{N}\right)\right)=\sup \left\{\beta \in(0, d): \int_{\mathbf{R}^{d}} \prod_{j=1}^{N} \operatorname{Re}\left(\frac{1}{1+\Psi_{j}(\xi)}\right) \frac{d \xi}{\|\xi\|^{d-\beta}}<+\infty\right\} .
$$

Here $\sup \varnothing:=0$.

Proof of Proposition 10.2. We choose and fix a $\beta \in(0, d)$, and assume that

$$
\mathrm{P}\left\{\mathscr{C}_{\beta}\left(\mathfrak{X}\left(\mathbf{R}_{+}^{N}\right)\right)>0\right\}>0,
$$

for there is nothing to prove otherwise.

Let us choose an integer $M \geq 1$ and a real number $\alpha \in(0,2]$ such that $\beta=d-M \alpha$. After enlarging the probability space, if need be, we may introduce $M$ i.i.d. isotropic stable processes $S_{1}, \ldots, S_{M}$-independent also of all $X_{j}$ 's - such that each $S_{j}$ has stability index $\alpha$. In this way, we can consider also the $M$-parameter additive Lévy process

$$
\mathfrak{S}(\boldsymbol{u}):=S_{1}\left(u_{1}\right)+\cdots+S_{M}\left(u_{M}\right) \text { for all } \boldsymbol{u}:=\left(u_{1}, \ldots, u_{M}\right) \in \mathbf{R}_{+}^{M} .
$$

defined on $\mathbf{R}^{d}$.

According to Theorem 7.2 of Khoshnevisan, Xiao, and Zhong (2003),

$$
\mathrm{P}\left\{\mathscr{C}_{\beta}\left(\mathfrak{X}\left(\mathbf{R}_{+}^{N}\right)\right)>0\right\}>0 \Leftrightarrow \mathrm{E}\left[\lambda_{d}\left(\mathfrak{X}\left(\mathbf{R}_{+}^{N}\right) \oplus \mathfrak{S}\left(\mathbf{R}_{+}^{M}\right)\right)\right]>0 .
$$

Because $\mathfrak{X} \oplus \mathfrak{S}$ is itself an $(N+M)$-parameter additive Lévy process, Proposition 10.11implies that the right-hand side of (10.10) is equivalent to

$$
\int_{\mathbf{R}^{d}}\left(\frac{1}{1+\|\xi\|^{\alpha}}\right)^{M} \prod_{j=1}^{N} \operatorname{Re}\left(\frac{1}{1+\Psi_{j}(\xi)}\right) d \xi<\infty .
$$

It remains to prove $\mathrm{P}\left\{\mathscr{C}_{\beta}\left(\mathfrak{X}\left(\mathbf{R}_{+}^{N}\right)\right)>0\right\}=1$.

The proof is similar to that of Proposition 2.8 in Khoshnevisan and Xiao (2005). Define the random probability measure $m$ on $\mathfrak{X}\left(\mathbf{R}_{+}^{N}\right)$ by

$$
\int_{\mathbf{R}^{d}} f(x) m(d x)=\int_{\mathbf{R}_{+}^{N}} f(\mathfrak{X}(\boldsymbol{t})) e^{-[\boldsymbol{t}]} d \boldsymbol{t},
$$

where $f: \mathbf{R}^{d} \rightarrow \mathbf{R}_{+}$denotes a Borel measurable function. It follows from Lemma 5.6 and the Fubini-Tonelli theorem that

$$
\mathrm{E}\left[\iint \frac{m(d x) m(d y)}{\|x-y\|^{\beta}}\right]=\frac{1}{(2 \pi)^{d}} \int_{\mathbf{R}^{d}} \mathrm{E}\left(\|\hat{m}(\xi)\|^{2}\right) \frac{d \xi}{\|\xi\|^{d-\beta}} .
$$


We may observe that

$$
\begin{aligned}
\mathrm{E}\left(\|\hat{m}(\xi)\|^{2}\right) & =\int_{\mathbf{R}_{+}^{N}} \int_{\mathbf{R}_{+}^{N}} \mathrm{E}\left[e^{-i \xi \cdot(\mathfrak{X}(\boldsymbol{t})-\mathfrak{X}(\boldsymbol{s}))}\right] e^{-[\boldsymbol{s}]-[\boldsymbol{t}]} d \boldsymbol{s} d \boldsymbol{t} \\
& =\prod_{j=1}^{N}\left[\int_{0}^{\infty} \int_{0}^{\infty} e^{-s_{j}-t_{j}-\left|s_{j}-t_{j}\right| \Psi_{j}\left(\operatorname{sgn}\left(s_{j}-t_{j}\right) \xi\right)} d s_{j} d t_{j}\right] .
\end{aligned}
$$

A direct computation shows that the preceding is equal to $K_{\Psi}(\xi)$; see also the proof of Lemma 3.4. Thanks to this and (10.11), the final integral in (10.13) is finite, whence it follows that $\mathscr{C}_{\beta}\left(\mathfrak{X}\left(\mathbf{R}_{+}^{N}\right)\right)>0$ almost surely.

Acknowledgements. A few years ago, Professor Jean Bertoin suggested to us a problem that is addressed by Theorem 1.4 of the present paper. We thank him wholeheartedly.

We have been writing several versions of this paper since April 2005. During the writing of one of this draft we have received many preprints by Dr. Ming Yang who, among other things, has independently discovered Propositions 10.1 and 10.2 and equation (10.7) of the present paper. His method combines the theory of Khoshnevisan, Xiao, and Zhong (2003) with a clever symmetrization idea, and is worthy of further investigation. We wish to thank Dr. Yang for communicating his work with us.

\section{REFERENCES}

Aizenman, Michael. 1985. The intersection of Brownian paths as a case study of a renormalization group method for quantum field theory, Comm. Math. Phys. 97(1-2), 91-110.

Albeverio, Sergio and Xian Yin Zhou. 1996. Intersections of random walks and Wiener sausages in four dimensions, Acta Appl. Math. 45(2), 195-237.

Berg, Christian and Gunnar Forst. 1975. Potential Theory on Locally Compact Abelian Groups, SpringerVerlag, New York.

Bertoin, Jean. 1999a. Subordinators: Examples and Applications, Lectures on Probability Theory and Statistics (Saint-Flour, 1997), pp. 1-91.

121. 1999b. Intersection of independent regenerative sets, Probab. Theory Related Fields 114(1), 97-

1996. Lévy Processes, Cambridge University Press, Cambridge.

Blumenthal, R. M. and R. K. Getoor. 1968. Markov Processes and Potential Theory, Academic Press, New York.

Csiszár, Imre. 1965. A note on limiting distributions on topological groups, Magyar Tud. Akad. Mat. Kutató Int. Közl. 9, 595-599 (1965) (English, with Russian summary).

Dellacherie, Claude and Paul-André Meyer. 1978. Probabilities and Potential, Vol. 29, North-Holland Publishing Co., Amsterdam.

Doob, Joseph L. 2001. Classical Potential Theory and Its Probabilistic Counterpart, Springer-Verlag, Berlin. Reprint of the 1984 edition. 
Dynkin, E. B. 1987. Self-intersection local times, occupation fields, and stochastic integrals, Adv. in Math. 65(3), 254-271.

1986. Generalized random fields related to self-intersections of the Brownian motion, Proc. Nat. Acad. Sci. U.S.A. 83(11), 3575-3576.

1985. Random fields associated with multiple points of the Brownian motion, J. Funct. Anal. 62(3), $397-434$.

1984a. Local times and quantum fields, Seminar on stochastic processes, 1983 (Gainesville, Fla., 1983), pp. 69-83.

1984b. Polynomials of the occupation field and related random fields, J. Funct. Anal. 58(1), 20-52. 1984c. Gaussian and non-Gaussian random fields associated with Markov processes, J. Funct. Anal. $\mathbf{5 5}(3), 344-376$.

1983a. Gaussian random fields and Gaussian evolutions, Theory and application of random fields (Bangalore, 1982), pp. 28-39.

. 1983b. Markov processes as a tool in field theory, J. Funct. Anal. 50(2), 167-187.

1981. Markov processes, random fields and Dirichlet spaces, Phys. Rep. 77(3), 239-247. New stochastic methods in physics.

1980. Markov processes and random fields, Bull. Amer. Math. Soc. (N.S.) 3(3), 975-999.

Dvoretzky, A., P. Erdős, and S. Kakutani. 1954. Multiple points of paths of Brownian motion in the plane, Bull. Res. Council Israel 3, 364-371.

1950. Double points of paths of Brownian motion in n-space, Acta Sci. Math. Szeged 12, 75-81.

Leopoldo Fejer et Frederico Riesz LXX annos natis dedicatus, Pars B.

Dvoretzky, A., P. Erdős, S. Kakutani, and S. J. Taylor. 1957. Triple points of Brownian paths in 3-space, Proc. Cambridge Philos. Soc. 53, 856-862.

Evans, Steven N. 1987a. Potential theory for a family of several Markov processes, Ann. Inst. H. Poincaré Probab. Statist. 23(3), 499-530 (English, with French summary).

1987b. Multiple points in the sample paths of a Lévy process, Probab. Theory Related Fields 76(3), $359-367$.

Farkas, Walter, Niels Jacob, and René L. Schilling. 2001. Function spaces related to continuous negative definite functions: $\psi$-Bessel potential spaces, Dissertationes Math. (Rozprawy Mat.) 393, 62.

Farkas, Walter and Hans-Gerd Leopold. 2006. Characterisations of function spaces of generalised smoothness, Ann. Mat. Pura Appl. (4) 185(1), 1-62.

Felder, G. and J. Fröhlich. 1985. Intersection properties of simple random walks: a renormalization group approach, Comm. Math. Phys. 97(1-2), 111-124.

Fitzsimmons, P. J. and Thomas S. Salisbury. 1989. Capacity and energy for multiparameter Markov processes, Ann. Inst. H. Poincaré Probab. Statist. 25(3), 325-350 (English, with French summary).

Fristedt, Bert. 1974. Sample Functions of Stochastic Processes with Stationary, Independent Increments, Advances in probability and related topics, Vol. 3, pp. 241-396.

Fukushima, Masatoshi, Yōichi Ōshima, and Masayoshi Takeda. 1994. Dirichlet Forms and Symmetric Markov Processes, Vol. 19, Walter de Gruyter \& Co., Berlin.

Getoor, R. K. 1990. Excessive Measures, Birkhäuser Boston Inc., Boston, MA.

Hawkes, John. 1984. Some geometric aspects of potential theory, Stochastic analysis and applications (Swansea, 1983), Lecture Notes in Math., vol. 1095, Springer, Berlin, pp. 130-154. 
- 1979. Potential theory of Lévy processes, Proc. London Math. Soc. (3) 38(2), 335-352.

1978a. Image and intersection sets for subordinators, J. London Math. Soc. (2) 17(3), 567-576.

1978b. Multiple points for symmetric Lévy processes, Math. Proc. Cambridge Philos. Soc. 83(1),

83-90.

1977. Local properties of some Gaussian processes, Z. Wahrscheinlichkeitstheorie und Verw. Gebiete 40(4), 309-315.

1976/77. Intersections of Markov random sets, Z. Wahrscheinlichkeitstheorie und Verw. Gebiete 37 (3), 243-251.

Hendricks, W. J. 1979. Multiple points for transient symmetric Lévy processes in $\mathbf{R}^{d}$, Z. Wahrsch. Verw. Gebiete 49(1), 13-21.

1973/74. Multiple points for a process in $R^{2}$ with stable components, Z. Wahrscheinlichkeitstheorie und Verw. Gebiete 28, 113-128.

Hendricks, W. J. and S. J. Taylor. 1979. Concerning some problems about polar sets for processes with stationary independent increments, preprint.

Hirsch, Francis. 1995. Potential theory related to some multiparameter processes, Potential Anal. 4(3), 245267.

Hirsch, Francis and Shiqi Song. 1999. Multiparameter Markov processes and capacity, Seminar on Stochastic Analysis, Random Fields and Applications (Ascona, 1996), pp. 189-200.

1996. Inequalities for Bochner's subordinates of two-parameter symmetric Markov processes, Ann.

Inst. H. Poincaré Probab. Statist. 32(5), 589-600 (English, with English and French summaries).

1995a. Markov properties of multiparameter processes and capacities, Probab. Theory Related

Fields 103(1), 45-71.

1995b. Symmetric Skorohod topology on n-variable functions and hierarchical Markov properties of n-parameter processes, Probab. Theory Related Fields 103(1), 25-43.

1995c. Une inégalité maximale pour certains processus de Markov à plusieurs paramètres. II, C. R.

Acad. Sci. Paris Sér. I Math. 320(7), 867-870 (French).

1995d. Une inégalité maximale pour certains processus de Markov à plusieurs paramètres. I, C. R.

Acad. Sci. Paris Sér. I Math. 320(6), 719-722 (French).

1994. Propriétés de Markov des processus à plusieurs paramètres et capacités, C. R. Acad. Sci.

Paris Sér. I Math. 319(5), 483-488 (French).

Hunt, G. A. 1958. Markoff processes and potentials. III, Illinois J. Math. 2, 151-213.

1957a. Markoff processes and potentials. I, II, Illinois J. Math. 1, 316-369.

1957b. Markoff processes and potentials. I, II, Illinois J. Math. 1, 44-93.

1956. Markoff processes and potentials, Proc. Nat. Acad. Sci. U.S.A. 42, 414-418.

Jacob, Niels. 2005. Pseudo Differential Operators and Markov Processes. Vol. III: Markov Processes and Applications, Imperial College Press, London.

2002. Pseudo Differential Operators 83 Markov Processes. Vol. II: Generators and Their Potential

Theory, Imperial College Press, London.

2001. Pseudo-Differential Operators and Markov Processes. Vol. I: Fourier Analysis and Semigroups, Imperial College Press, London. 
Jacob, Niels and René L. Schilling. 2005. Function spaces as Dirichlet spaces (about a paper by W. Mazya and J. Nagel). Comment on: "On equivalent standardization of anisotropic functional spaces $H^{\mu}\left(\mathbf{R}^{n}\right)$ " (German) [Beiträge Anal. No. 12 (1978), 7-17; MR0507094], Z. Anal. Anwendungen 24(1), 3-28.

Kahane, Jean-Pierre. 1985. Some Random Series of Functions, 2nd ed., Cambridge University Press, Cambridge.

Kakutani, Shizuo. 1944a. On Brownian motions in n-space, Proc. Imp. Acad. Tokyo 20, 648-652. 1944b. Two-dimensional Brownian motion and harmonic functions, Proc. Imp. Acad. Tokyo 20, $706-714$.

Kesten, Harry. 1969. Hitting Probabilities of Single Points for Processes with Stationary Independent Increments, Memoirs of the American Mathematical Society, No. 93, American Mathematical Society, Providence, R.I.

Khoshnevisan, Davar. 2003. Intersections of Brownian motions, Expo. Math. 21(2), 97-114. 1999. Brownian sheet images and Bessel-Riesz capacity, Trans. Amer. Math. Soc. 351(7), 26072622.

2002. Multiparameter Processes: An Introduction to Random Fields, Springer-Verlag, New York. Khoshnevisan, Davar, Narn-Rueih Shieh, and Yimin Xiao. 2007. Hausdorff dimension of the contours of symmetric additive Lévy processes, Probab. Th. Rel. Fields, to appear.

Khoshnevisan, Davar and Yimin Xiao. 2004. Additive Lévy processes: capacity and Hausdorff dimension, In: Proc. of Inter. Conf. on Fractal Geometry and Stochastics III., Progress in Probability, vol. 57, pp. 62-100, Birkhäuser. 2005. Lévy processes: capacity and Hausdorff dimension, Ann. Probab. 33(3), 841-878.

Khoshnevisan, Davar, Yimin Xiao, and Yuquan Zhong. 2003. Measuring the range of an additive Lévy process, Ann. Probab. 31(2), 1097-1141.

Lawler, Gregory F. 1989. Intersections of random walks with random sets, Israel J. Math. 65(2), 113-132. 1985. Intersections of random walks in four dimensions. II, Comm. Math. Phys. 97(4), 583-594. 1982. The probability of intersection of independent random walks in four dimensions, Comm. Math. Phys. 86, no. 4, 539-554.

Le Gall, Jean-François. 1992. Some properties of planar Brownian motion, École d'Été de Probabilités de Saint-Flour XX — 1990, Lecture Notes in Math., vol. 1527, Springer, Berlin, pp. 111-235.

1987. Le comportement du mouvement brownien entre les deux instants où il passe par un point double, J. Funct. Anal. 71(2), 246-262.

Le Gall, Jean-François, Jay S. Rosen, and Narn-Rueih Shieh. 1989. Multiple points of Lévy processes, Ann. Probab. 17 (2), 503-515.

Lévy, Paul. 1940. Le mouvement brownien plan, Amer. J. Math. 62, 487-550 (French).

Marcus, Michael B. and Jay Rosen. 1999b. Multiple Wick product chaos processes, J. Theoret. Probab. $12(2), 489-522$.

1999a. Renormalized self-intersection local times and Wick power chaos processes, Mem. Amer. Math. Soc. 142 (675).

Masja, Wladimir and Jürgen Nagel. 1978. Über äquivalente Normierung der anisotropen Funktionalräume $H^{\mu}\left(\mathbf{R}^{n}\right)$, Beiträge Anal., no. 12, 7-17 (German).

Mattila, Pertti. 1995. Geometry of Sets and Measures in Euclidean Spaces, Cambridge University Press, Cambridge. 
Orey, Steven. 1967. Polar sets for processes with stationary independent increments, Markov Processes and Potential Theory (Proc. Sympos. Math. Res. Center, Madison, Wis., 1967), pp. 117-126.

Pemantle, Robin, Yuval Peres, and Jonathan W. Shapiro. 1996. The trace of spatial Brownian motion is capacity-equivalent to the unit square, Probab. Theory Related Fields 106(3), 379-399.

Peres, Yuval. 1999. Probability on Trees: An Introductory Climb, Lectures on probability theory and statistics (Saint-Flour, 1997), pp. 193-280. 1996a. Remarks on intersection-equivalence and capacity-equivalence, Ann. Inst. H. Poincaré Phys. Théor. 64(3), 339-347 (English, with English and French summaries).

1996b. Intersection-equivalence of Brownian paths and certain branching processes, Comm. Math. Phys. 177 (2), 417-434.

Ren, Jia Gang. 1990. Topologie p-fine sur l'espace de Wiener et théorème des fonctions implicites, Bull. Sci. Math. 114(2), 99-114 (French, with English summary).

Röckner, Michael. 1993. General Theory of Dirichlet Forms and Applications, In: Dirichlet forms (Varenna, 1992), Lecture Notes in Math., vol. 1563, Springer, Berlin, pp. 129-193.

Rogers, L. C. G. 1989. Multiple points of Markov processes in a complete metric space, Séminaire de Probabilités, XXIII, pp. 186-197.

Rosen, Jay. 1984. Self-intersections of random fields, Ann. Probab. 12(1), 108-119. 1983. A local time approach to the self-intersections of Brownian paths in space, Comm. Math. Phys. 88(3), 327-338.

Salisbury, Thomas S. 1996. Energy, and intersections of Markov chains, Random discrete structures (Minneapolis, MN, 1993), IMA Vol. Math. Appl., vol. 76, Springer, New York, pp. 213-225.

1992. A low intensity maximum principle for bi-Brownian motion, Illinois J. Math. 36(1), 1-14. 1988. Brownian bitransforms, Seminar on Stochastic Processes, 1987 (Princeton, NJ, 1987), Progr.

Probab. Statist., vol. 15, Birkhäuser Boston, Boston, MA, pp. 249-263.

Sato, Ken-iti. 1999. Lévy Processes and Infinitely Divisible Distributions, Cambridge University Press, Cambridge. Translated from the 1990 Japanese original, Revised by the author.

Schoenberg, I. J. 1938. Metric spaces and positive definite functions, Trans. Amer. Math. Soc. 44, 522-536.

Slobodeckiı̌, L. N. 1958. S. L. Sobolev's spaces of fractional order and their application to boundary problems for partial differential equations, Dokl. Akad. Nauk SSSR (N.S.) 118, 243-246 (Russian).

Tongring, Nils. 1988. Which sets contain multiple points of Brownian motion?, Math. Proc. Cambridge Philos. Soc. 103(1), 181-187.

Ville, Jean. 1942. Sur un problème de géométrie suggéré par l'étude du mouvement brownien, C. R. Acad. Sci Paris 215, 51-52 (French).

Walsh, John B. 1986. Martingales with a Multidimensional Parameter and Stochastic Integrals in the Plane, Lectures in probability and statistics (Santiago de Chile, 1986), Lecture Notes in Math., vol. 1215, Springer, Berlin, pp. 329-491.

Westwater, J. 1980. On Edwards' model for long polymer chains, Comm. Math. Phys. 72(2), 131-174.

Westwater, John. 1981. On Edwards' model for polymer chains. II. The self-consistent potential, Comm. Math. Phys. 79(1), 53-73.

1982. On Edwards' model for polymer chains. III. Borel summability, Comm. Math. Phys. 84(4), $459-470$. 
Wolpert, Robert L. 1978. Local time and a particle picture for Euclidean field theory, J. Funct. Anal. 30(3), $341-357$.

Davar Khoshnevisan: Department of Mathematics, The University of Utah, 155 S. 1400 E. SAlt Lake City, UT 84112-0090

E-mail address: davar@math.utah.edu

$U R L:$ http://www.math.utah.edu/〜davar

Department of Statistics and Probability, A-413 Wells Hall, Michigan State University, EAST LANSing, MI 48824

E-mail address: xiao@stt.msu.edu

$U R L:$ http://www.stt.msu.edu/ ${ }^{x i a o y i m i}$ 